\title{
Roadmap on Magnetoelectric Materials and Devices
}

Xianfeng Liang ${ }^{1}$, Alexei Matyushov ${ }^{1,3}$, Patrick Hayes ${ }^{2}$, Viktor Schell ${ }^{2}$, Cunzheng Dong ${ }^{1}$, Huaihao Chen ${ }^{1}$, Yifan He ${ }^{1}$, Alexandria Will-Cole ${ }^{1}$, Eckhard Quandt ${ }^{2}$, Pedro Martins ${ }^{4,5}$, Jeffrey McCord ${ }^{2}$, Marisa Medarde ${ }^{6}$, Senentxu Lanceros-Méndez ${ }^{4,7,8}$, Sebastiaan van Dijken ${ }^{9}$, Nian X. Sun ${ }^{1}$ and Jordi Sort ${ }^{10,11}$

${ }^{1}$ Department of Electrical and Computer Engineering, Northeastern University, Boston, MA 02115, USA

${ }^{2}$ Institute for Materials Science, Kiel University, Kiel, 24143, Germany

${ }^{3}$ Department of Physics, Northeastern University, Boston, MA 02115, USA

${ }^{4}$ Centro/Departamento de Física, Universidade do Minho, Braga 4710-057, Portugal

5 IB-S Institute of Science and Innovation for Sustainability, Universidade do Minho, 4710-057, Braga, Portugal

${ }^{6}$ Laboratory for Multiscale Materials Experiments, Paul Scherrer Institute, 5232 Villigen PSI, Switzerland

7 BCMaterials, BCMaterials, Basque Center for Materials, Applications and Nanostructures, UPV/EHU Science Park, 48940 Leioa, Spain

8 IKERBASQUE, Basque Foundation for Science, 48009 Bilbao, Spain

${ }^{9}$ NanoSpin, Department of Applied Physics, Aalto University School of Science, P. O. Box 15100, FI-00076 Aalto, Finland

10 Departament de Física, Universitat Autònoma de Barcelona, E-08193 Cerdanyola del Vallès, Spain

11 Institució Catalana de Recerca i Estudis Avançats (ICREA), Pg. Lluís Companys 23, E-08010 Barcelona, Spain

Corresponding authors: N. Sun (Email: n.sun@northeastern.edu); J. Sort (Email: jordi.sort@uab.cat)

\begin{abstract}
The possibility to tune the magnetic properties of materials with voltage (converse magnetoelectricity) or to generate electric voltage with magnetic fields (direct magnetoelectricity) has opened new avenues in a large variety of technological fields, ranging from information technologies to healthcare devices and including a great number of multifunctional integrated systems such as mechanical antennas, magnetometers, RF tunable inductors, etc., which have been realized due to the strong strain-mediated magnetoelectric (ME) coupling found in ME composites. The development of single-phase multiferroic materials (which exhibit simultaneous ferroelectric and ferromagnetic or antiferromagnetic orders), as well as progress in other ME mechanisms, such as electrostatic surface charging or magneto-ionics (voltage-driven ion migration) have a large potential to boost energy efficiency in spintronics and magnetic actuators. This paper focuses on existing ME materials and devices and reviews the state of the art in their performance. The most recent progress on different ME devices based on
\end{abstract}


ME heterostructures is presented, including ME antennas, sensors, and others. Significant effort and rapid development of mechanical ME antennas has been observed over the past few years. These mechanically actuated ME antennas are miniaturized by 1-2 orders compared to conventional antenna size. Ultra-sensitive magnetic sensors, which are based on simple $\mathrm{ME}$ composites, have been considered to be next-generation magnetic sensors and promising alternatives to conventional magnetic sensors due to their very good detectivity $\left(<\mathrm{pT} / \mathrm{Hz}^{1 / 2}\right)$ at low frequencies. Other ME devices reviewed in this paper include RF tunable inductors with high inductance tunability and quality (Q) factor; non-reciprocal microelectromechanical system (MEMS) bandpass filters with dual $\mathrm{H}$ - and $\mathrm{E}$-field tunability; passive isolators and gyrators in the low frequency (LF) range; and magnetoelectric random-access memories for low-power data storage. All these compact and lightweight ME devices are also promising for future biomedical and wireless applications. Finally, some open questions and future directions where the community might be headed are provided.

\section{Introduction}

Magnetoelectricity refers to the influence of an applied magnetic field on the electric polarization of certain materials (direct magnetoelectric (ME) effect) or, contrarywise, the influence of external electric field on the magnetization (converse ME effect). The effect was first postulated in 1894 [1] and experimentally observed for the first time in 1960 [2] (Fig. 1). So far, ME effects have been observed in (i) single-phase "multiferroics" (a term coined in 1994 to designate materials with two or three types of simultaneous ferroic orders, such as ferroelectricity and ferromagnetism [3]), and (ii) piezoelectric/magnetostrictive or piezomagnetic/electrostrictive composite heterostructures [4]. The primary requirement for $M E$ effects in single-phase materials is the presence of adequate structural and symmetry conditions that allow the coexistence of spontaneous long-range magnetic and electric orders. Most single-phase multiferroic materials are rather complex oxides that exhibit their properties mainly at low temperatures. Nonetheless, ME effects have been reported at room temperature (RT) in $\mathrm{Cr}_{2} \mathrm{O}_{3}$ and $\mathrm{BiFeO}_{3}$. These materials are both antiferromagnetic, with virtually zero net magnetization [5]. Heterogeneous multiferroics, comprising ferroelectric (FE)/ferromagnetic (FM) bilayers, are the most common for practical applications. Examples of $\mathrm{FE}$ materials are some perovskites $\mathrm{ABO}_{3}$ $(A=$ alkaline/alkali earth metal, $\mathrm{Pb} ; \mathrm{B}=\mathrm{Ti}, \mathrm{Zr}, \mathrm{Nb}$, etc.) or polymers like Poly(vinylidene fluoridetrifluoroethylene $\mathrm{P}(\mathrm{VDF}-\mathrm{TrFE})$ ). $\mathrm{FM}$ include magnetostrictive alloys (e.g., FeGa), spinel $\mathrm{MFe}_{2} \mathrm{O}_{4}$ oxides ( $M=$ transition metal) or $M$-type hexagonal ferrites $\mathrm{AFe}_{12} \mathrm{O}_{19}(\mathrm{~A}=$ alkali earth metal)]. An alternative strategy for engineering enhanced voltage-driven ME effects at RT is to introduce indirect coupling, mediated via strain, between piezoelectric and magnetostrictive materials. Strain coupling requires good mechanical matching between the two constituent phases. For this reason, the effects are mainly observed in composite films or patterned structures where the crystallographic orientation and interfacial roughness are accurately controlled. In the latter, strain gradient effects (eventually, flexoelectricity [6]) can add to homogeneous strains to boost magnetoelectricity. Besides multiferroics, novel procedures to tailor magnetism with voltage have emerged during recent years [7-9], such as electric surface charging (to tune the magnetic anisotropy of ultrathin-film or mesoporous alloys) and magneto-ionics (i.e., voltage-driven ion diffusion/intercalation). 


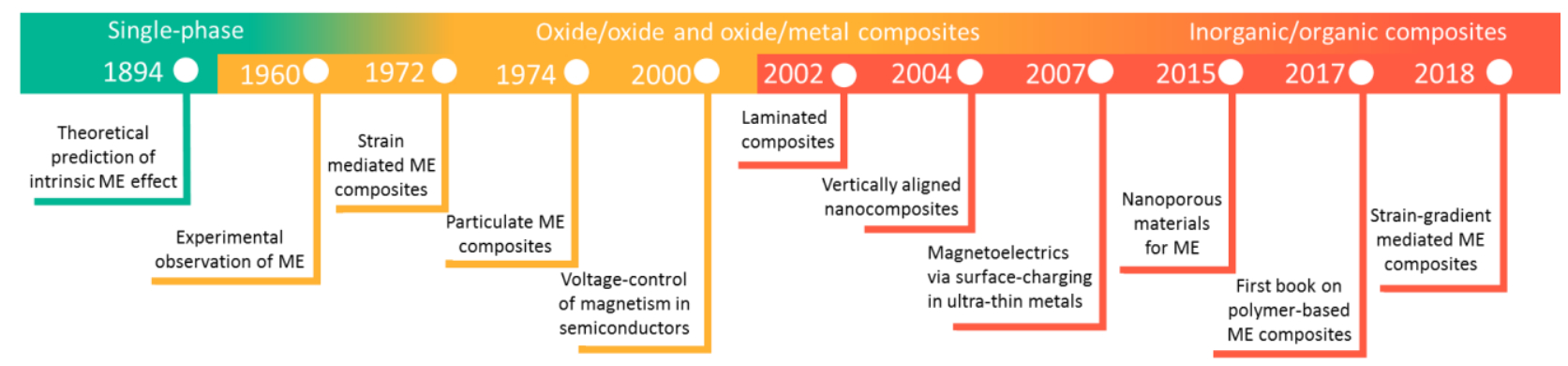

Fig. 1. Timeline showing the emergence of various types of ME materials. Adapted from Ref. 10.

The number of patents and scientific publications on ME materials have rapidly increased during the last two decades (see Fig. 2(a)). Accordingly, ME effects in bulk materials and thick films have been utilized in a myriad of conventional applications: magnetic field sensors (automotive, robotics), energy harvesters, radio-frequency/microwave devices, radar telecommunications, and transducers, amongst others. The total market based on ME materials has become several thousand M€/year (www.marketsandmarkets.com), with most of the patents in the period of 2000-2018 having been issued in China, Japan, and USA (Fig. 2(b)).
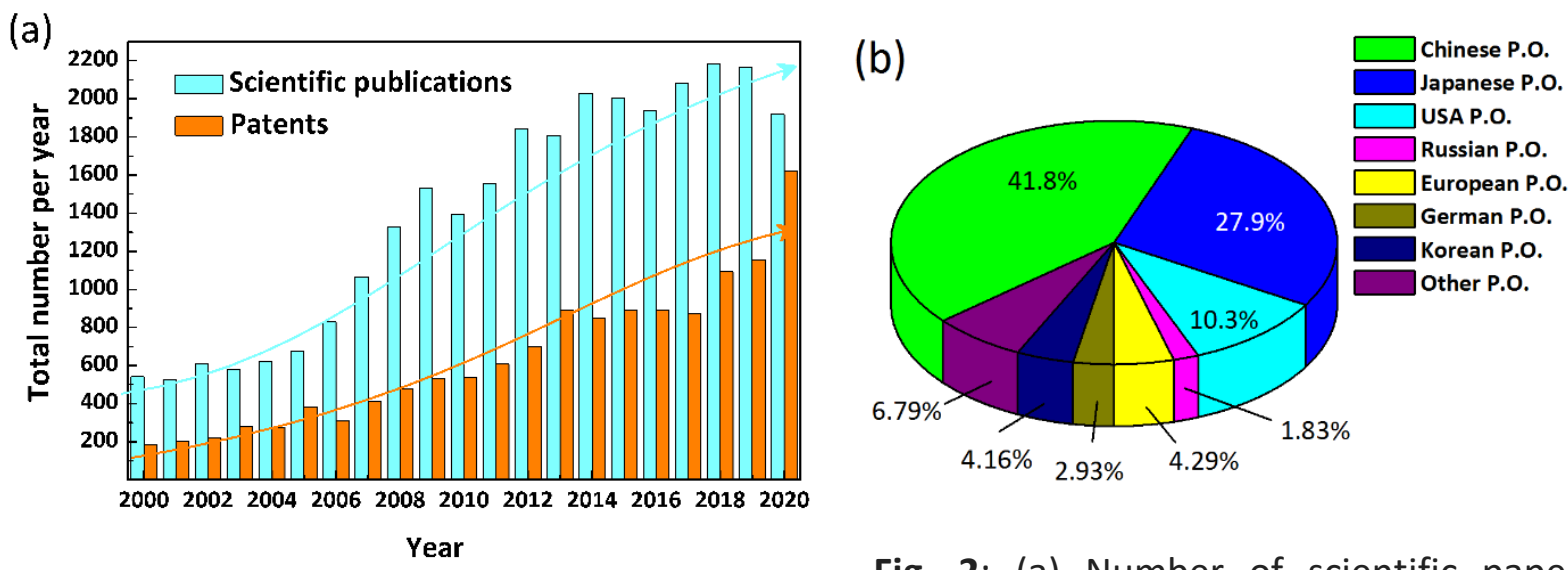

Fig. 2: (a) Number of scientific papers (according to the 'ISI Web of Science') and patents per year (according to 'Google Patents'), using the keyword 'magnetoelectric', 'magneto-electric' or 'multiferroic', during the period 2000-2020. (b) Geographical distribution of the patents issued during this period by the different existing patents offices (P.O.) (extracted from 'Google Patents').

Such utilization of the ME effect was enabled by the attainment of ME heterostructures with strong ME coupling [11-23], which results in large ME coefficients. Given the two kinds of ME coupling-direct and converse ME effects-two coefficients have been defined to quantify the coupling strength: $\alpha_{\text {Direcct }}=\partial P / \partial H$ and $\alpha_{\text {Converse }}=\partial M / \partial E$, respectively. Only with large ME coefficients can the ME effect be useful for applications, so this breakthrough has led to excellent further progress on novel ME materials and devices. Consequently, a great number of multifunctional ME devices, such as mechanical antennas [24-29], magnetic sensors [30-34], tunable inductors [35-38] and filters [39-42], etc. have been developed. These are summarized and categorized in Table I based on the physical mechanism and the type of ME coupling. 
Compared to conventional devices, these voltage-tunable ME devices have the benefits of compactness, low weight, etc.

Table I. Physical mechanisms of various ME devices. Adapted from [19].

\begin{tabular}{lll}
\hline ME devices & ME coupling & Physical mechanisms \\
\hline Magnetic sensors, gyrators & Direct ME coupling & H control of P \\
$\begin{array}{l}\text { Voltage tunable inductors, } \\
\text { filters }\end{array}$ & Converse ME coupling & E control of $\mu$ \\
Mechanical antennas & $\begin{array}{l}\text { Direct and converse } \\
\text { coupling }\end{array}$ & $\begin{array}{l}\text { Interaction between electric } \\
\text { and magnetic phases }\end{array}$ \\
\hline
\end{tabular}

The additional category of mechanical ME antennas is included in Table I due to the high interest that this topic that has attracted recently. Notably, a maximum communication distance of $120 \mathrm{~m}$ for a Very Low Frequency (VLF) communication system using a pair of ME antennas has been achieved. Furthermore, ME antennas based on piezoelectric/magnetostrictive heterostructures with acoustic actuation, and which are smaller by one to two orders of magnitude than state-ofthe-art compact antennas, have been demonstrated to operate at very-high frequency (VHF, $60 \mathrm{MHz}$ ) and ultra-high frequency (UHF, $2.525 \mathrm{GHz}$ )

With the advent of nanotechnology, new applications incorporating nanostructured ME materials (thin films, mesoporous alloys, nano-patterned structures) are emerging. These encompass two innovative domains: information technologies (e.g., random access memories (ME-RAM) [43], voltage-controlled magnetic logic devices [44], high-density magnetic data storage [45], magnonics [46]) and advanced healthcare technologies (e.g., wireless deep neural stimulation therapies [47] or ME drug delivery [48,49]). In the following review, we describe recent progress on different types of multiferroic materials as well as devices based on $\mathrm{ME}$ heterostructures that exploit ME effects in various ways.

\subsection{Note on magnetic sensor detection limits and terminology}

A major subset of ME devices discussed in this review includes magnetic field sensors and ME antennas. Given the purpose and applications of such devices, one of their defining properties is how small of a magnetic field signal such a device can reasonably detect. However, on this point, some notable inconsistencies exist in the body of work of the communities studying various types of magnetometers. Thus, a word of caution is in order about the methods and terminology related to detection of magnetic fields.

There are actually two separate issues: first, how to quantify the smallest magnetic field a sensor or antenna can detect, and second, what term(s) to use for such a measure. Mainly, two ways have been used to report the detection limit: in dimensions of [field] or in dimensions of 
[field]/vHz. The former approach adopts a certain fixed set of parameters relevant to the sensor (such as the voltage sustaining the sensor operation) and then measures the sensor output at decreasing magnetic field strengths until the output signal flatlines, thereby establishing the minimum field detected under these parameters. The latter method is superior in terms of quantifying the intrinsic properties of the sensor, which are independent of experimental parameters, but is more complex, requiring a measurement of the power spectral density (PSD) of the intrinsic noise floor of the sensor. The square root of the PSD of noise is then divided by the sensor's sensitivity (defined as the change in sensor output signal per change in magnetic field) to give a standardized measure of the detection limit [50-52]; thus, the presence of the noise spectrum in this ratio explains the dimension of $\mathrm{Hz}^{-1 / 2}$ in the resulting quantity. This characterization is more suitable for making comparisons across sensors that have different structures and detection mechanisms and may be tested under different parameters. In particular, under the first method, the lowest detectable magnetic field signal can be arbitrarily improved by methods like increasing acquisition time or averaging. However, the PSD is already normalized by acquisition time [50], thus removing this source of arbitrariness.

The second issue is the lack of agreement on terminology. Whereas it is common to find magnetic sensor performance reported in terms of [field] $/ \mathrm{vHz}$, this result has been variously referred to as "field equivalent noise" [50], "equivalent magnetic noise" [53], "magnetic noise" [26], "detectivity" [51], "limit of detection" [54], "minimum detectable signal" [52], "sensitivity" [55], and perhaps other names. It would benefit the magnetic sensor community to adopt a shared standard of terms. To this end, as well as to unify the discussion of results covered in this review, we propose the following. First, we suggest that "sensitivity" be reserved for quantifying the change in the sensor's output signal per unit of magnetic field, [56] and that "magnetic noise" be used to designate the noise from magnetization fluctuations and other magnetic effects. Second, we propose a new term: "minimum detected field" (MDF) to designate the first kind of result discussed above, given in dimensions of the magnetic field only (previously, "limit of detection" has been used in this case [32], but this may create confusion when the same term is used for both types of measurements). For the second type of quantity, [field]/vHz, we like "detectivity" for its concision and the implication that it is not an absolute detection limit but rather an estimate or metric [51], [52] to indicate the kind of field strengths that the sensor can be reasonably expected to detect. Thus, these are the definitions employed in the text to follow except where the authors were unable to alter the labels within the original figures that have been reproduced here.

\section{Magnetoelectric materials}

\subsection{Single phase multiferroics}

Although implementation in real devices requires in general materials than can be stabilized as thin films, the precise determination of the crystal and magnetic structures and the comprehension of the intrinsic ME coupling mechanism requires the use of bulk materials, preferably in high-quality single-crystalline form. Unfortunately, and despite an important number of material discoveries in the last decade, the number of single-phase multiferroics 
potentially suitable for device applications remains very limited. An ideal material should display switchable, strongly coupled electric and magnetic order parameters at room temperature (RT). It also has to be a decent insulator, and the different states of the order parameters should be large in value and robust enough to be read and manipulated with presently available techniques. Such a material was not known in 2013, when the state of the art on RT multiferroics was first summarized in a review by J.F. Scott [57], and it is still missing seven years after in spite of intense activity in the field [58-63]. On the other hand, the research carried out during the last decade allowed to better characterize $[63,64]$ and (in some cases) to improve the properties of some of the best candidates. A new promising family and a novel mechanism to create RT multiferroicity were also identified. Here we briefly review such advances, compare the different candidates, and suggest some ideas for further development.

\section{a) $\mathrm{BiFeO}_{3}$}

\section{Crystal structure}

The rhombohedrically-distorted perovskite $\mathrm{BiFeO}_{3}$ was for long time the only material featuring both, (antiferro)magnetic order and a large ferroelectric polarization at room temperature. Moreover, its relatively simple distorted perovskite structure is an advantage for theoretical studies, making it at the same time compatible with most of the materials used in thin film oxide electronics. As a result, it has concentrated most of the activity in the field during the last 20 years, i.e., since the seminal paper of Hill (now Spaldin) [65]. The number of both, experimental and theoretical studies devoted to this material is huge, and it is still in the leading position in spite of the discovery of other potential candidates. Here we summarize the main characteristics of the bulk material as benchmark for the remaining candidates, and refer to the excellent review by Catalan and Scott [66] and references therein for further reading.

\section{Transition temperatures, magnetization and polarization}

Bulk $\mathrm{BiFeO}_{3}$ is a good ferroelectric with a high Curie temperature $T_{C}=1103 \mathrm{~K}$ due to the stereochemical activity of the $\mathrm{Bi}^{3+}$ s lone pair and large polarization $\left(P \sim 100 \mu \mathrm{C} / \mathrm{cm}^{2}\right.$ along the $<111>_{\text {pseudocubic }}$ directions and $R \sim 6 \times 10^{10} \Omega \mathrm{cm}$ in good quality single crystals $[67,68]$. It is also longperiod ( $62 \mathrm{~nm}$ ) cycloidal antiferromagnet with a slightly incommensurate magnetic propagation vector $\mathbf{k}$ parallel to the $[1,0,-1]_{\text {pseudocubic }}$ direction $[69,70]$. The $\mathrm{Fe}^{3+}$ magnetic moments rotate in the plane defined by $\mathbf{k}$ and $\mathbf{P}$, as well as a weak ferromagnetic component that cancels due to the cycloid's rotation. The Néel temperature $T_{N}=643 \mathrm{~K}$ is also high, so, antiferromagnetism and ferroelectricity coexist at RT with a comfortable margin of several hundred degrees.

\section{Magnetoelectric effects}

Although $\mathrm{BiFeO}_{3}$ is a good ferroelectric, it is not ferromagnetic. Moreover, antiferromagnetism and ferroelectricity appear at different temperatures, and are originated by different mechanisms. Interestingly, bulk $\mathrm{BiFeO}_{3}$ displays substantial $\mathrm{ME}$ effects, although their strength and tensor characteristics are highly dependent of the nature of the applied field (H or E). Moderate $(H<10 T)$ magnetic fields cause only a marginally weak increase of $\mathbf{P}$ proportional to $H^{2}$, but for $H>20 T$ the coupling between $\mathbf{P}$ and $\mathbf{H}$ becomes linear and coinciding with a sign reversal of $\mathbf{P}[71]$. This is due to the replacement of the spin cycloid by an incommensurate conical 
magnetic order [72] which is accompanied by a small increase of the magnetization. The application of electric fields, more interesting for applications, has also an impact in the magnetism. This was first demonstrated by Lebeugle and co-workers, which showed that a $71^{\circ}$ rotation of $\mathbf{P}$ around $\mathbf{k}$ results in a rotation of the spiral plane around the magnetic propagation vector by the same value [70]. Such observation was an important milestone that opened the way to the first experimental demonstration of electric control of the magnetization at RT by the same group. For this purpose, Lebeugle et al. grow a layer of a strong ferromagnet (permalloy Py) on the surface of a bulk $\mathrm{BiFeO}_{3}$ crystal, and showed that the Py easy magnetization axis could be toggled with an electric field due to the exchange coupling between the two materials through their interface [73]. These findings have been crucial for the interpretation of subsequent work in $\mathrm{BiFeO}_{3}$ thin films, and for the design of spintronic devices exploiting the abovementioned coupling effects $[74,75]$. They also redirected the attention towards antiferromagnetic ferroelectrics (more abundant than their ferromagnetic counterparts), showing that an electric control of the magnetization may also be possible with this kind of materials.

\section{b) Aurivilius phases}

\section{Crystal structure}

Aurivillius compounds are a family of layered oxides with general formula of $\left(\mathrm{Bi}_{2} \mathrm{O}_{2}\right)^{2+}\left(\mathrm{A}_{n-1}\right.$ $\left.\mathrm{B}_{n} \mathrm{O}_{3 n+1}\right)^{2-}$, where perovskite-type blocks alternate fluorite-type $\left(\mathrm{Bi}_{2} \mathrm{O}_{2}\right)^{2+}$ slabs, and $n$ designates the number of $\mathrm{ABO}_{3}$ perovskite units per half-unit cell [76]. As $\mathrm{BiFeO}_{3}$, they are in general good ferroelectrics with high resistivity. They are also known by their low fatigue $[77,78]$. and high Curie temperatures, which can reach values higher than $1000 \mathrm{~K}$. Their high polarization originates from the displacements of the ions in the $\left(\mathrm{Bi}_{2} \mathrm{O}_{2}\right)^{2+}$ layers with respect to those in the perovskite layers, which are usually non-magnetic. It is oriented along the a crystal axis -i.e., perpendicular to the stacking direction, and in general, it is hard to saturate [76].

Since the first report of simultaneous ferroelectric and antiferromagnetic behaviour in $\mathrm{Bi}_{5} \mathrm{FeTi}_{3} \mathrm{O}_{15}(n=4), \mathrm{Bi}_{6} \mathrm{Fe}_{2} \mathrm{Ti}_{3} \mathrm{O}_{18}(n=5)$ and $\mathrm{Bi}_{9} \mathrm{Fe}_{5} \mathrm{Ti}_{3} \mathrm{O}_{27}(n=8)$ [79], the main strategy to promote these strong ferroelectrics into multiferroics has been the incorporation of magnetic ions to thein B-sites of the perovskite units. Unfortunately, their nature and concentration are strongly limited by the electric neutrality of the structure. For example, trivalent $\mathrm{A}$-site cations such a $\mathrm{Bi}^{3+}$ require average $\mathrm{B}$-cation valences ranging from $4+(n=3)$ to $3+(n=\infty)$. Large-moment cations such as $\mathrm{Fe}^{3+}$ or $\mathrm{Co}^{2+}$ can thus only be incorporated together with the necessary amounts of higher valence cations (such as $\mathrm{Co}^{4+}, \mathrm{Ti}^{4+}, \mathrm{Ta}^{5+}, \mathrm{Nb}^{5+}$ or $\mathrm{W}^{6+}$ ). Since many of them are non-magnetic, their presence in large amounts may substantially disrupt the connectivity of the magnetic ion network, preventing the occurrence of long-range magnetic ordering [80]. This problem is less acute in Aurivilius phases with a large number of perovskite layers, but these materials are more prone to display structural imperfections that may leading to leakage currents. Their large unit cells, with $c \approx 16,25,32,41,50$ and $58 \AA$ for $n=1,2,3,4,5$ and 6, respectively, are also a limiting factor for modeling due to the huge number of atoms per unit cell [80], and make the growth of epitaxial, stacking fault-free thin film growth challenging.

Transition temperatures, magnetization and polarization 
In the recent years, most of the research activity has been concentrated in the $n=4 \mathrm{Fe} / \mathrm{Co}$ solid solutions $\mathrm{Bi}_{5} \mathrm{Fe}_{1-\mathrm{x}} \mathrm{Co}_{\mathrm{x}} \mathrm{Ti}_{3} \mathrm{O}_{15}$, either in ceramic, polycrystalline thin film and (less often) singlecrystalline form. Several studies have reported the coexistence of ferroelectricity and weak ferromagnetism in these materials at RT with remnant polarizations up to $\approx 16 \mu \mathrm{C} / \mathrm{cm}^{2}$, remnant magnetizations up to $\approx 7.8 \mathrm{memu} / \mathrm{g}$, and ferromagnetic Curie temperatures up to $\approx 750 \mathrm{~K}$ [ 81 84]. Slightly improved magnetic properties have been reported for $\mathrm{Bi}_{7} \mathrm{Fe}_{2} \mathrm{NiTi}_{3} \mathrm{O}_{21}(n=6)$, with a ferromagnetic Curie temperature $T_{c}=773 \mathrm{~K}$ and substantially larger remnant magnetization (1.32 emu/g) at RT [85]. This material also presents a sizable remnant polarization $\left(\approx 5 \mu \mathrm{C} / \mathrm{cm}^{2}\right)$, but suffers from more leakage than the Ni-free compound, an observation attributed to a larger number of defects. At this point it is worth mentioning that the presence of intrinsic ferromagnetism in these materials has been questioned by some studies due to the possible presence of small amounts of impurities with large RT magnetization such as $\mathrm{Co}_{3-\mathrm{x}} \mathrm{Fe}_{\mathrm{x}} \mathrm{O}_{4}$ spinel ferrites, whose presence is difficult to detect by standard techniques such as laboratory x-ray diffraction [86]. This point is of utmost importance, and calls for additional efforts in the synthesis and material characterization.

\section{Magnetoelectric effects}

Early reports of linear ME effects in Aurivilius phases can be tracked back to the eighties. For $\mathrm{Bi}_{5} \mathrm{FeTi}_{3} \mathrm{O}_{15}$ the reported direct $\mathrm{ME}$ coefficients $\left(0.1\right.$ to $\left.11 \mathrm{mV} \mathrm{cm}^{-1} \mathrm{Oe}^{-1}\right)$ were however very small, although larger than in $\mathrm{BiFeO}_{3}\left(0.064 \mathrm{mV} \mathrm{cm}^{-1} \mathrm{Oe}^{-1}\right)$ [87-89]. More recent reports on this material and other Aurivilius phases with different $n$ and different magnetic atoms seem to confirm this tendency [90], although the number or reports signaling the presence of ME coupling remains very limited [81-83]. An interesting development that may inspire further investigations was the magnetic-field-induced ferroelectric switching in a grain of a polycrystalline $\mathrm{Bi}_{6} \mathrm{Ti}_{2.8}$ $\mathrm{Fe}_{1.52} \mathrm{Mn}_{0.68} \mathrm{O}_{18}$ film [86], a rather unexpected observation given the difficulties for reaching polarization saturation in this class of materials [76]. The opposite effect, i.e., an electric-field induced reversal of the magnetization has not been reported to the best of our knowledge.

\section{c) Hexaferrites}

\section{Crystal structure}

Hexaferrites are a family of Fe-containing oxides of hexagonal or rhombohedral symmetry whose structures are built by stacking three different structural units along the c-axis: the so-called $\boldsymbol{S}$ $\left[\mathrm{M}^{2+} \mathrm{Fe}_{5} \mathrm{O}_{8}\right], \boldsymbol{R}\left[(\mathrm{Ba}, \mathrm{Sr}) \mathrm{Fe}_{6} \mathrm{O}_{11}\right]^{2-}$, and $\boldsymbol{T}\left[(\mathrm{Ba}, \mathrm{Sr}) \mathrm{Fe}_{8} \mathrm{O}_{14}\right]$ blocks, where $\mathrm{M}^{+2}$ designates a small $3 \mathrm{~d}$ divalent cations such as $\mathrm{Co}^{+2}, \mathrm{Ni}^{+2}$ or $\mathrm{Zn}^{+2}$. The resulting compounds are characterized by large unit cells that can be classified in six different families: $M, W, Y, Z, X$ and $U$-type, with $c \approx 23,33$, $43,52,84$ and $113 \AA$, respectively [59]. As in the case of the Aurivillius phases, this structural complexity makes the growth of single crystal, and epitaxial, stacking fault-free thin film growth challenging. Hence, most materials are only available in polycrystalline form [91].

Hexaferrites are in general insulating when iron is present in a single oxidation state [91]. However, electron and/or hole doping though chemical substitution or $\mathrm{O}$ vacancies, stacking faults -quite frequent owing to their large unit cells-, or accumulation of charges in the grain boundaries can lead to a substantial increase in their electrical conductivity [91]. 
Transition temperatures, magnetization and polarization

Compared with $\mathrm{BiFeO}_{3}$ and Aurivilius phases, the most distinctive characteristic of hexaferrites is that they are not ferroelectric. However, they are ferrimagnetic at RT, with elevated Curie temperatures $(>600 \mathrm{~K}$ ) and large saturation magnetization ( $\approx 30$ to $90 \mathrm{emu} / \mathrm{g}$ ) either along a single direction $(\mathrm{M})$, within a plane $(\mathrm{Y}, \mathrm{Z}, \mathrm{U})$ or along a cone $(\mathrm{W}, \mathrm{X})$ [91]. Interestingly, it was demonstrated by Kimura and co-workers that they can develop electric polarization under the application of moderate magnetic fields [92], in some cases at RT [93]. This is due to the changes in the magnetic structures induced by the magnetic field, not fully characterized due to their enormous complexity, but believed to develop cycloidal components that can couple to the lattice by inducing acentric charge displacements in order to minimize the relativistic antisymmetric product $D_{i j} \mathbf{S}_{\mathbf{i}} \mathbf{X} \mathbf{S}_{\mathbf{j}}$ of neighbouring spins $\boldsymbol{S}_{\mathbf{i}}$ and $\boldsymbol{S}_{\mathbf{j}}[59]$.

In the Z-hexaferrite $\mathrm{Sr}_{3} \mathrm{CO}_{2} \mathrm{Fe}_{24} \mathrm{O}_{41}$, the material with the best RT figures to date, its high resistivity at $300 \mathrm{~K}\left(\approx 10^{9} \Omega \mathrm{cm}\right)$ enabled to measure a polarization $P \approx 0.001 \mu \mathrm{C} / \mathrm{cm}^{2}$ under a magnetic field of only $0.2 T$ [93]. Similar observations have been reported for other hexaferrites belonging to different families [91]. Unfortunately, in most of them polarization measurements could be only conducted at cryogenic temperatures due to their leaky nature. If this point could be solved, either through improved ceramic preparation, or with the growth of defect-free single crystals, their structural flexibility may allow also to improve the RT polarization, directly linked to the details of the magnetic order. Ab initio calculations indicated that the exchange interactions are strongly dependent on the details of the Fe distribution in the structure, suggesting that progress in the control of this parameter could also result in better ferroelectric properties [94].

\section{Magnetoelectric effects}

The magnetic-field induced polarization has in general a non-linear dependence with $\mathbf{H}$ due to the complex $\mathrm{T}$-H low-field phase diagrams of most of these materials. In the case of $\mathrm{Sr}_{3} \mathrm{CO}_{2} \mathrm{Fe}_{24} \mathrm{O}_{41}$, $\alpha$ is maximum at $\approx 3 \mathrm{mT}$, reaching a value of $\approx 0.0063 \mathrm{mV} \mathrm{cm}^{-1} \mathrm{Oe}^{-1}$, about 10 times smaller than that of $\mathrm{BiFeO}_{3}$. For this material, resonant soft-x-ray microdiffraction was recently used to image the two types of magnetic domains associated respectively to the ferrimagnetic and spiral components of its RT transverse conical magnetic structure. Moreover, a simultaneous inversion of both types of domains by reversing the applied magnetic field of $\sim 0.3 T$ was observed [95]. The opposite effect, i.e., an electric-field induced magnetization has not been reported to the best of our knowledge.

\section{d) Layered Cu-Fe perovskites}

\section{Crystal structure}

Compared to Aurivilius phases, hexaferrites, and even with $\mathrm{BiFeO}_{3}$, the most distinctive characteristic of the layered perovskite family $\mathrm{RACuFeO}_{5}(\mathrm{R}=4 \mathrm{f}$ lanthanide, $\mathrm{A}=\mathrm{Ba}$ and/or $\mathrm{Sr}$ ) is its structural simplicity. Their tetragonal unit cell contains two pseudocubic perovskite units stacked along the $\mathrm{c}$ axis [96], where the A-cations ( $\mathrm{R}$ and $\mathrm{Ba}$ ) order in layers due to their very different ionic radii. The $\mathrm{B}$-positions are occupied by $\mathrm{Fe}$ and $\mathrm{Cu}$, in square pyramidal coordination due the presence of ordered $\mathrm{O}$ vacancies. But contrarily to $\mathrm{Cu}$ and $\mathrm{Ba}$, they are usually disordered. 
Although most past studies have been conducted in ceramic samples, the growth of large $\mathrm{YBaCuFeO}_{5}$ single crystals has been recently reported [97]. Thin films, not reported to date, are probably easy to growth owing to the layered nature. Moreover, their in-plane lattice parameter is comparable to several commonly used perovskite substrates.

The transport properties of these materials have not been investigated in detail, but the available studies indicate semiconducting behavior with RT resistances between $10^{6}-10^{10} \Omega$ [98]. Although in general they are perfectly stoichiometric, $\mathrm{O}$ can be incorporated in the lattice if the sizes of the two A-site cations are too similar, resulting in a fast increase of the conductivity [99].

Transition temperatures, magnetization and polarization

In spite of their simple crystal structure (Fig. 3), Cu/Fe layered perovskites are the only known materials featuring magnetic cycloidal phases stable beyond RT in absence of an external magnetic field (in hexaferrites, where the magnetic order is still under debate, such phases seem to appear only under the application of a magnetic field). However, what makes Cu/Fe layered perovskites truly unique is the tunability of their spiral ordering temperature $T_{\text {spiral, }}$, that can be easily and continuously changed between $\approx 150$ and $\approx 400 \mathrm{~K}$ by adjusting the size of the A-site cations and the degree of $\mathrm{Cu} / \mathrm{Fe}$ chemical disorder in the B-sites [100]. Surprisingly, this last variable has a gigantic, positive impact in the stability of the magnetic spiral. In $\mathrm{YBaCuFeO}_{5}$, the most investigated among these materials, a $6 \%$ difference in $\mathrm{Cu} / \mathrm{Fe}$ occupational disorder can promote $T_{\text {spiral }}$ from 154 to $310 \mathrm{~K}$ [101], and this temperature can be further increased up to $\approx$ $375 \mathrm{~K}$ by combining maximal $\mathrm{Cu} / \mathrm{Fe}$ disorder with the exchange of $40 \%$ of $\mathrm{Ba}$ by $\mathrm{Sr}$ [100]. Interestingly, the cycloidal component of the magnetic spiral increases with the $T_{\text {spiral }}$ value, suggesting that the materials with the highest spiral transition temperature could also show the largest polarization [100].

An direct consequence of the layered perovskites structural simplicity (just 9 atoms per unit cell) is that they are easier to model. The huge impact of $\mathrm{Cu} / \mathrm{Fe}$ disorder in the stability of the spiral phase has indeed inspired the formulation of a novel, disorder-based local frustration model able to predict the periodicity and the ordering temperature of the spiral phase from the magnetic exchange constants and the degree of disorder [102,103]. Their predictions have been successfully verified in the case of $\mathrm{Cu} /$ Fe layered perovskites, suggesting that it could serve as basis for the design of other materials with $T_{\text {spiral }}$ phases at RT and beyond. 

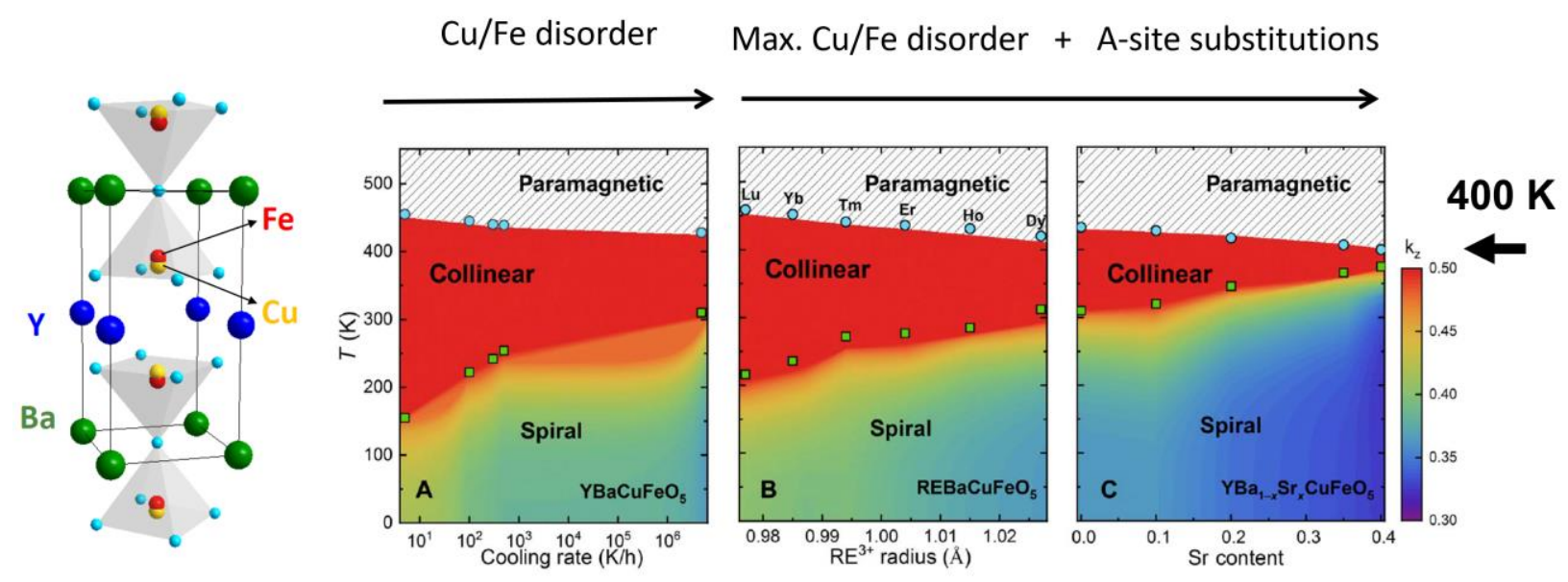

Fig. 3. Crystal structure of the layered perovskite $\mathrm{YBaCuFeO}_{5}$, and evolution of the stability region of its magnetic spiral phase using different tuning strategies. a) Cooling rate of the last annealing used to modify the degree of $\mathrm{Cu} / \mathrm{Fe}$ disorder in the bipyramidal sites. b) Replacement of $\mathrm{Y}^{3+}$ by a trivalent rare earth cation with different ionic radius. c) Partial replacement of $\mathrm{Ba}^{2+}$ by $\mathrm{Sr}^{2+}$. In b) and c) the samples were cooled using a very fast cooling rate in order to reach a high degree of $\mathrm{Cu} / \mathrm{Fe}$ disorder. In all panels, the color scale indicates the value of the incommensurate component $k_{z}$ of the spiral magnetic propagation vector $\mathbf{k}=\left(1 / 21 / 2 k_{z}\right)$ (adapted from ref. 100).

Contrarily to their crystal and magnetic structures, the dielectric and ferroelectric properties of these materials have not been investigated in detail. Given the existence of cycloidal components in (nearly) all the materials investigated, spontaneous polarization is expected to appear below $T_{\text {spiral. }}$ This has been indeed observed in $\mathrm{YBaCuFeO}_{5}$ ceramic samples by three different groups $[96,104,105]$ although in some cases the non-exact coincidence of $T_{\text {spiral }}$ with the polarization setup suggests the presence of leakage. On these reports, pyrocurrent measurements give saturation polarization values up to $0.6 \mu \mathrm{C} / \mathrm{cm}^{2}$, close to the $1 \mu \mathrm{C} / \mathrm{cm}^{2}$ considered the minimum for applications, and significantly larger than the values reported for hexaferrites. As in the case of $\mathrm{BiFeO}_{3}$, leakage problems are expected to be less severe in single crystals. Measurements on crystals will be also needed to determine the polarization direction, theoretically predicted to be in the ab plane, but presently unknown. P-V ferroelectric hysteresis cycles have not been reported, at least to our knowledge.

As $\mathrm{BiFeO}_{3}, \mathrm{Cu} / \mathrm{Fe}$ layered perovskites are antiferromagnets without net spontaneous magnetization $[96,106]$. However, given the (usually strong) response of magnetic spirals to external magnetic fields, low-field. incommensurate conical phases with small ferromagnetic components, similar to those reported for hexaferrites could exist in these materials as well.

\section{Magnetoelectric effects}

The existence of $\mathrm{ME}$ coupling in $\mathrm{YBaCuFeO}_{5}$ was first reported by Kundys et al. [104], who observed a 85\% decrease of the polarization in ceramic samples after applying a 9T magnetic field. The same team reported also a nearly linear increase of the relative dielectric permittivity with $\mathbf{H}$ for fields up to $14 \mathrm{~T}$ that reached values of $\approx+0.25 \%$ at $14 \mathrm{~T}$ in the vicinity of $T_{\text {spiral }}$. 
Magnetocapacitance effects have been also reported by Luo et al. [107], but they are negative and about 10 times larger than those reported by Kundys. A possible origin for these discrepancies can be the huge dependence of the magnetic order with the $\mathrm{Cu} / \mathrm{Fe}$ disorder (and hence with the preparation method). Measurements on well characterized single crystals will be necessary to clarify the nature of the observed $\mathrm{ME}$ effects in $\mathrm{YBaCuFeO}_{5}$.

Table II. Single-phase multiferroics where RT ME effects have been reported of have high chances to exist.

\begin{tabular}{|c|c|c|c|c|c|c|}
\hline $\begin{array}{l}\text { Family and best } \\
\text { performing } \\
\text { materials }\end{array}$ & $\begin{array}{l}\text { Origin Ferro- } \\
\text { electricity }\end{array}$ & $\begin{array}{l}\text { Ferroelectric } \\
\text { temperature } \\
\text { range }(K)\end{array}$ & $\begin{array}{l}\text { (Anti) } \\
\text { ferromagnetic } \\
\text { temperature } \\
\text { range }(\mathrm{K})\end{array}$ & $\begin{array}{l}\text { Maximum } \\
\text { polarizatio } \\
n \\
\left(\mu \mathrm{C} / \mathrm{cm}^{2}\right)\end{array}$ & Main advantages & Main drawbacks \\
\hline $\mathrm{BiFeO}_{3}$ & $\mathrm{Bi}^{3+\prime}$ s lone pair & $\mathrm{T}_{\mathrm{C}}=1103[66]$ & $T_{N}=643[66]$ & $\approx 100[67]$ & $\begin{array}{l}\text { - Large polarization } \\
\text { - High magnetic and ferro- } \\
\text { electric ordering } \\
\text { temperature } \\
\text { - Magnetoelectric coupling }\end{array}$ & $\begin{array}{l}\text { - Antiferromagnetic } \\
\text { - Complex domain } \\
\text { structure }\end{array}$ \\
\hline $\begin{array}{l}\text { Aurivilius phases } \\
\mathrm{Bi}_{5} \mathrm{Fe}_{1-x} \mathrm{~B}_{x} \mathrm{Ti}_{3} \mathrm{O}_{15} \\
(\mathrm{~B}=\mathrm{Co}, \mathrm{Mn})\end{array}$ & $\mathrm{Bi}^{3+\prime}$ s lone pair & $<1030[81-84]$ & $<750[81-84]$ & $\approx 16[81-84]$ & $\begin{array}{l}\text { - Large polarization } \\
\text { - High ferroelectric ordering } \\
\text { temperatures }\end{array}$ & $\begin{array}{l}\text { - Structural complexity } \\
\text { - Ferromagnetism } \\
\text { questioned } \\
\text { - Magnetoelectric coupling } \\
\text { not fully characterized }\end{array}$ \\
\hline $\begin{array}{l}\text { Hexaferrites } \\
\mathrm{Sr}_{3} \mathrm{CO}_{2} \mathrm{Fe}_{24} \mathrm{O}_{41}\end{array}$ & Cycloidal spiral & $<400[108]$ & $<670$ [93] & $\begin{array}{l}\approx 0.001 \\
\text { (under } 0.2 \mathrm{~T} \\
\text { at RT) [93] }\end{array}$ & $\begin{array}{l}\text { - Large magnetization } \\
\text { - High magnetic ordering } \\
\text { temperatures } \\
\text { - Magnetoelectric } \\
\text { coupling }\end{array}$ & $\begin{array}{l}\text { - Structural complexity } \\
\text { - Magnetic order no fully } \\
\text { characterized } \\
\text { - Polarization very small, } \\
\text { and only under magnetic } \\
\text { field }\end{array}$ \\
\hline $\begin{array}{l}\text { Layered perovskites } \\
\mathrm{YBa}_{1-\mathrm{x}} \mathrm{Sr}_{\mathrm{x}} \mathrm{CuFeO}_{5}\end{array}$ & Cycloidal spiral & $\begin{array}{c}<400 \text { (potential) } \\
<230 \text { (reported) } \\
{[104]}\end{array}$ & $<400[100]$ & $\approx 0.6[104]$ & $\begin{array}{l}\text { - Very simple crystal structure } \\
\text { - High, easily tunable spiral } \\
\text { ordering temperature } \\
\text { - Theoretical model } \\
\text { available for property } \\
\text { improvement }\end{array}$ & $\begin{array}{l}\text { - Antiferromagnetic } \\
\text { - Ferroelectric properties } \\
\text { and magnetoelectric } \\
\text { coupling not fully } \\
\text { characterized } \\
\text { - Polarization small }\end{array}$ \\
\hline
\end{tabular}

Future trends in single-phase multiferroics

To date, the most successful mechanisms towards RT multiferroicity in bulk materials have been i) the insertion of magnetic ions in RT strong ferroelectrics, and ii) the stabilization of magnetic cycloids at RT using magnetic fields, chemical substitutions and/or or chemical disorder. Examples of the first mechanism are $\mathrm{BiFeO}_{3}$ - to date the only single phase multiferroic seriously considered for applications - and the Aurivilius phases, whereas hexaferrites and Cu-Fe layered perovskites are examples of the second. Although none of these mechanisms has produced yet an ideal RT bulk multiferroic (see Table II) the different material candidates have still room for improvement. However, this will not happen in the near future without substantial efforts in bulk material's synthesis, characterization and modeling. The new possibilities for electric and magnetic domain imaging, summarized in two recent reviews $[63,109]$ may contribute to these efforts, hopefully helping to transfer the most promising materials and phenomena to thin films. 
In parallel, it is important to pursue the investigation of other multiferroic mechanisms (such as charge order), and to check experimentally novel theoretical predictions $[60,62]$.

\subsection{Inorganic multiferroic composites}

ME multiferroic composites comprising inorganic ferroelectric and magnetic phases that indirectly couple through engineered interfaces have been studied intensively for several decades. The abundance of materials exhibiting robust electric or magnetic order parameters at room temperature offers flexibility in the optimization of coupling strengths, the design and fabrication of practical devices, and the tailoring of material properties for new application areas. In ME composites, interface coupling produces two attractive product properties; magnetic-fieldto-voltage conversion and electric-field control of magnetism.

\section{Magnetic-field-to-voltage conversion}

Initial works on multiferroic composites focused mainly on the engineering of $M E$ voltage coefficients $\left(\alpha_{\mathrm{E}}=d E / d H\right)$ in bulk samples. ME coupling in bulk composites is based on strain mediation between phase-separated magnetostrictive and piezoelectric effects. Besides appropriate material selection, the phase connectivity, interface integrity, and electrical resistance of the composite greatly influence the coupling strength. In bulk form, 0-3 particulate composites and 2-2 laminates have been studied extensively (see Fig. 4). Particulate composites consisting of microscale mixtures of perovskite ferroelectrics $\left(\mathrm{BaTiO}_{3}, \mathrm{PbTiO}_{3}, \mathrm{~Pb}(\mathrm{Zr}, \mathrm{Ti}) \mathrm{O}_{3}\right)$ and ferrites ( $\mathrm{CoFe}_{2} \mathrm{O}_{4}, \mathrm{NiFe}_{2} \mathrm{O}_{4}$, and others) exhibit $\alpha_{\mathrm{E}}$ values in the range $1-500 \mathrm{mV} \mathrm{cm}^{-1} \mathrm{Oe}^{-1}$ at low frequency [110-118], while larger effects are induced at mechanical resonance [119-122]. Leakage through the (semi)conducting ferrite phase limits the ME coefficient in particulate composites. While strategies are available to partly overcome this detriment (e.g. by the dispersion of core-shell particles [123-126]), the largest upturns in ME coupling strength have been realized by the layering of ferroelectric and magnetic components into 2-2 bulk laminates. Ceramic laminates made of the same perovskite ferroelectrics and ferrites typically exhibit one order of magnitude larger $\alpha_{\mathrm{E}}$ values compared to their particulate counterpart [127-129]. The largest $\mathrm{ME}$ coupling coefficients $\left(\alpha_{\mathrm{E}}>5 \mathrm{~V} \mathrm{~cm}^{-1} \mathrm{Oe}^{-1}\right)$ are attained in laminates comprising ferromagnetic metals such as Terfenol-D ( $\mathrm{Tb}_{1-\mathrm{x}} \mathrm{D} \mathrm{y}_{\mathrm{x}} \mathrm{Fe}_{2}$ alloys with giant magnetostriction) [130132] or Metglas (amorphous Fe-based alloys with high magnetic permeability) [132, 133]. Cosintering and adhesive bonding are popular methods for the fabrication of ceramic and alloybased laminates. Following advances in thin-film growth techniques, the development of various multiferroic nanocomposites took off about 15 years ago. Nanocomposites fabricated by physical deposition or chemical solution processing offer improved control over the interface structure and lattice strain. Vertically aligned 1-3 nanocomposites (VANs) in which 1D magnetic spinel nanopillars ( $\mathrm{CoFe}_{2} \mathrm{O}_{4}, \mathrm{NiFe}_{2} \mathrm{O}_{4}, \mathrm{MgFe}_{2} \mathrm{O}_{4}$, and others) are embedded in a ferroelectric perovskite matrix $\left(\mathrm{BaTiO}_{3}, \mathrm{PbTiO}_{3}, \mathrm{BiFeO}_{3}\right)$ and 2-2 bilayers or multilayers have been studied most. VANs are often prepared by self-assembly during simultaneous deposition of two immiscible magnetic and ferroelectric compounds $[134,135]$. Because of the large vertical interface area between the two

phases and limited clamping by the substrate, VANs are predicted to exhibit large ME coupling coefficients [136-139]. Yet, leakage currents through the conducting spinel pillars restrict the 
experimentally derived values to $\alpha_{\mathrm{E}}=10-200 \mathrm{mV} \mathrm{cm}^{-1} \mathrm{Oe}^{-1}$ [140-142]. In recent years, studies on multiferroic VANs have focused on the templating of nanopillars into ordered arrays and the integration of VANs with silicon [143]. ME coupling effects in bilayers or multilayers are restricted by substrate clamping. Despite this limitation, thin-film composites such as AIN/(Fego $\left.\mathrm{CO}_{10}\right)_{78} \mathrm{Si}_{12} \mathrm{~B}_{10}$ [144] and $\mathrm{BaTiO}_{3} / \mathrm{CoFe} / \mathrm{BaTiO}_{3}$ [145] have been shown to exhibit $\mathrm{ME}$ coefficients up to $5 \mathrm{~V} \mathrm{~cm}^{-1} \mathrm{Oe}^{-1}$ at low frequency and several hundred $\mathrm{V} \mathrm{cm}^{-1} \mathrm{Oe}^{-1}$ at the mechanical resonance frequency.

Research on ME composites with optimized ME voltage coefficients is mature and more details on materials, composite structures, fabrication methods, etcetera, can be found in other reviews $[11,16-18,20,146-153]$. In the last decade, the field has gravitated towards the implementation of practical devices. Applications of magnetic-field-to-voltage conversion include magnetic field sensors [154], current sensors [155], and energy harvesters [156].

(a)

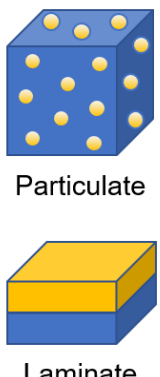

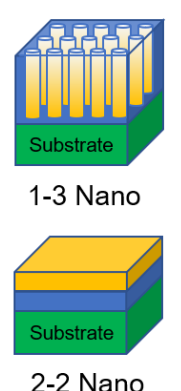

2-2 Nano (b)

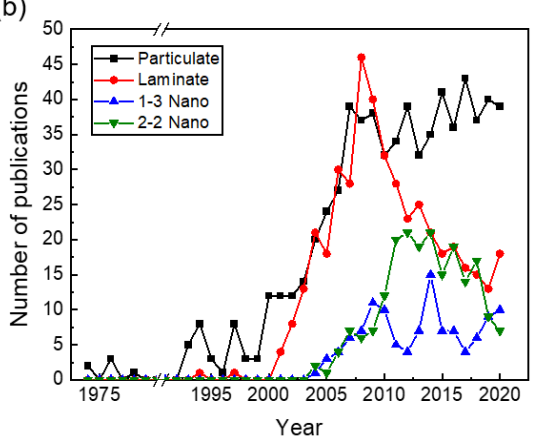

(c)

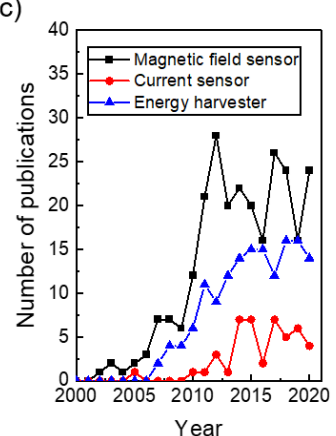

(d)

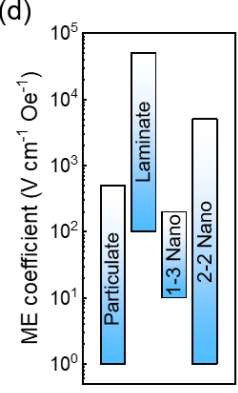

Fig. 4. (a) Schematic illustration of magnetoelectric composite structures: bulk 0-3 particulates, bulk 2-2 laminates, vertically aligned 1-3 nanostructures (1-3 Nano), and 2-2 bilayers or multilayers grown on a substrate (2-2 nano). (b) Number of publications on magnetoelectric composites as a function of publishing year. (c) Number of publications on various applications of magnetic-field-to-voltage conversion in magnetoelectric composites. (d) Range of magnetoelectric coefficients reported for the different types of magnetoelectric composites. The data in (b) and (c) are extracted from the SCOPUS database.

\section{Electric-field control of magnetism}

Control of magnetism through the application of electric fields across multiferroic composites has been studied extensively during the last 15 years [7, 8, 18, 147, 150, 157, 158]. Macroscopically, the effect is quantified by the converse ME coefficient ( $\alpha=\mu_{0} d M / d E$ ). While coupling strengths have been reported for many inorganic material systems, comparisons are not straightforward because of differences in the underlying coupling mechanism and the plurality of magnetic parameters that change during actuation. Besides strain mediation, polarization switching in a ferroelectric layer can alter the properties of a thin magnetic film via electrostatic charge modulation or electronic hybridization at the interface. Exchange coupling between a single-phase antiferromagnetic multiferroic layer (e.g. $\mathrm{BiFeO}_{3}$ ) and a ferromagnetic film provides another attractive means for electric-field control of magnetism. Depending on the ME coupling mechanism and magnetic material, applying an electric field across a multiferroic composite can change the three magnetic energies, saturation magnetization $(M)$, exchange 
interaction, and magnetic anisotropy $(K)$, as well as other magnetic properties including the transition temperature $\left(T_{\mathrm{C}}\right)$, exchange bias field $\left(H_{\mathrm{ex}}\right)$, spin polarization $(\mathrm{SP})$, magnetoresistance $(\mathrm{MR})$, and ferromagnetic resonance (FMR) frequency. These wide-ranging effects are of technological importance for applications in magnetic memories, logic devices, and tunable microwave components. For information technology, low-energy dissipation is one of the key selling points of electric-field control of magnetism compared to current-driven devices based on spin-transfer or spin-orbit torques. From the perspective of energy usage, the induced change of magnetism per applied voltage rather than electric field is a more relevant figure-of-merit for the efficiency of ME coupling [8].

A popular method for studying strain-mediated electric-field control of magnetism involves the growth of magnetic films onto piezoelectric or ferroelectric single-crystal substrates. The most used piezoelectric material is $(1-x) \mathrm{Pb}(\mathrm{Mg} 1 / 3 \mathrm{Nb} 2 / 3) 03-x \mathrm{PbTiO} 3$ (PMN-PT), which is a relaxor ferroelectric with excellent electromechanical and piezoelectric properties for compositions near the morphotropic phase boundary $(0.25 \leq x \leq 0.35)$ [159]. Transfer of piezostrain from PMN-PT has been utilized to tune the magnetic properties of manganites [160-162], ferrites [163-166], and metallic ferromagnets with in-plane [167-171] and perpendicular magnetic anisotropy [172, 173]. PMN-PT crystals exhibit a butterfly-shaped voltage-strain curve that is single-valued at zero electric field. The response of a magnetic film grown on top of PMN-PT mimics the shape of the piezostrain curve [160]. To establish non-volatile magnetic switching for ME memory applications, symmetry-breaking mechanisms have been proposed and demonstrated. Among them are the use of competing magnetic anisotropies [174-176], partial poling of the piezoelectric layer [168, 177, 178], structural phase transitions in PMN-PT [179], ferroelastic domain switching [180], and fast strain dynamics [181-183]. Strain transfer from PMN-PT (or PZT [163, 184-186] and PZN-PT $[163,174,180])$ substrates to magnetic oxides has been shown to modify the magnetization, transition temperature, magnetoresistance, magnetic anisotropy or FMR. Most studies involving ferromagnetic metals report the manipulation of magnetic anisotropy or related changes in FMR.

Ferroelectric $\mathrm{BaTiO}_{3}$ substrates are another prominent choice for attaining strain-mediated electric-field control of magnetism. Contrary to PMN-PT and other piezoelectrics, $\mathrm{BaTiO}_{3}$ single crystals comprise regular $90^{\circ}$ polarization domains at room temperature. The ferroelectric domains are therefore also ferroelastic. Local strain transfer during magnetic film growth on top of $\mathrm{BaTiO}_{3}$ substrate has been shown to produce fully correlated ferromagnetic/ferroelectric stripe domains via inverse magnetostriction [188-191]. The strain-coupled magnetic domain patterns are characterized by a regular modulation of magnetic anisotropy and strong pinning of magnetic domain walls onto the ferroelectric domain boundaries. Applying an electric field across $\mathrm{BaTiO}_{3}$ abruptly switches the polarization or it gradually modifies the ferroelectric domain pattern via lateral domain wall motion. Strain-coupling translates these changes into alterations of magnetic anisotropy, the magnetic domain size, or the location of domain walls in the magnetic film. Electric-field control of magnetism based on ferroelastic strain transfer from $\mathrm{BaTiO}_{3}$ substrates has been demonstrated for several magnetic materials, including $\mathrm{La}_{0.67} \mathrm{Sr}_{0.33} \mathrm{MnO}_{3}$ [192], Fe [193-196], $\mathrm{Ni}$ [197-199], CoFe [188, 189], $\mathrm{Fe}_{3} \mathrm{O}_{4}$ [200], FeGa [201, 202], and FeRh $[203,204]$. Most studies on these multiferroic composites report electric-field-induced changes of magnetization or magnetic anisotropy. In FeRh films, a strain-driven reversible 
transition between antiferromagnetic and ferromagnetic phases has been demonstrated just above room temperature. The strain that is transferred from a $\mathrm{BaTiO}_{3}$ substrate to a magnetic film is up to $1.1 \%$ at room temperature, which is one order of magnitude larger than the strains produced by piezoelectric crystals. Efficient strain transfers from ferroelastic domain switching in $\mathrm{BaTiO}_{3}$ can therefore be used to electrically manipulate relatively thick films, as demonstrated by full domain correlations in $150 \mathrm{~nm} \mathrm{CoFeB} / \mathrm{BaTiO}_{3}$ [191].

While strain-mediated ME coupling between magnetic films or nanostructures and piezo- or ferroelectric substrates offers electric-field control of magnetic properties, strong magnetic responses in these composites require large voltages $(20-500 \mathrm{~V})$. For reversible effects at only a few volts and on-chip device integration, the substrates need to be replaced by thin-film piezoor ferroelectrics. Growing oxide films onto a substrate is often not effective as clamping restricts the ME coupling in 2-2 nanocomposites. Despite this limitation, electrodes on top of relatively thick piezoelectric films have been used to control magnetism in patterned nanostructures via non-uniform in-plane strains [205]. Besides, patterning of micro- or nanostructures that contain both functional layers of the multiferroic composite provides a viable alternative. Multilayer growth through a porous anodic alumina template has been used to fabricate such heterostructures [206, 207].

Direct electric-field effects on magnetism have been explored mostly in gated structures with non-polar dielectric oxides. In such structures, screening of the electric field by spin-polarized charge carriers near the interface of a thin magnetic film alters the magnetic moment or magnetic anisotropy. A similar phenomenon is obtained when an electric field switches the perpendicular polarization in a ferromagnetic/ferroelectric bilayer. The ME coupling coefficient is larger for ferroelectric oxides than non-polar oxides because of the proportionality between the screeningcharge density in the magnetic film and the dielectric constant of the gate oxide $\left(\varepsilon_{r}=100-1000\right.$ in ferroelectrics). Another polarization switching effect originates from electronic hybridization between $3 d$ transition metal atoms at the interface. Both effects occur at the interface, which limits the thickness of magnetic films that an electric field can manipulate. First-principle calculations based on density functional theory indicate that the magnetic moment of Fe atoms at a $\mathrm{Fe} / \mathrm{BaTiO}_{3}$ interface changes by about $5 \%$ due to a shift in the Fe-Ti bond length during ferroelectric polarization reversal [208]. Similar effects have been calculated for $\mathrm{Co}_{2} \mathrm{MnSi} / \mathrm{BaTiO}_{3}$ [209], $\mathrm{Fe} / \mathrm{PbTiO}_{3}$ [210-212], $\mathrm{Fe}_{3} \mathrm{O}_{4} / \mathrm{BaTiO}_{3}$ [213], and $\mathrm{Co} / \mathrm{PbZr}_{\mathrm{x}} \mathrm{Ti}_{1-\mathrm{x}} \mathrm{O}_{3}$ [214]. Electric-field effects based on charge modulation are particularly prominent in perovskite manganites due to strong lattice-spin-charge coupling. The accumulation or depletion of charge carriers near the interface of a manganite film changes the hole doping concentration, a parameter that is normally controlled by substitution of $\mathrm{La}$ ions of the $\mathrm{LaMnO}_{3}$ parent compound with alkaline earth ions. As a result, polarization reversal in an adjacent ferroelectric film can change the magnetic and electronic ground state of manganites when the material is positioned near one of its phase transitions. Electrostatic control of manganite films has been demonstrated by theoretical calculations and experiments on multiferroic bilayers, most notably $\mathrm{La}_{1-x} \mathrm{~A}_{x} \mathrm{MnO}_{3} / \mathrm{BaTiO}_{3}(\mathrm{~A}=\mathrm{Sr}$, $\mathrm{Ca}$, or $\mathrm{Ba}$ ) [215-218] and $\mathrm{La}_{1-\mathrm{x}} \mathrm{A}_{\mathrm{x}} \mathrm{MnO}_{3} / \mathrm{PbZr}_{\mathrm{x}} \mathrm{Ti}_{1-\mathrm{x}} \mathrm{O}_{3}$ [219-223] with $\mathrm{A}=\mathrm{Sr}$, $\mathrm{Ca}$, or $\mathrm{Ba}$. Besides effects on magnetization, magnetic anisotropy, magnetoresistance, ordering temperature, and magnetic phase transitions, ferroelectric switching at a magnetic interface can also induce a 
change in spin polarization [224-227], an effect that could be exploited in multilevel storage devices utilizing magnetic tunnel junctions with ferroelectric barriers [228].

Many single-phase multiferroic materials are antiferromagnetic (Section 2.1). Intrinsic coupling between the ferroelectric polarization and the antiferromagnetic spin lattice in such materials can therefore be utilized to electrically control the exchange bias interaction with a ferromagnetic film. Multiferroic materials that have been explored for studies on exchange bias include $\mathrm{YMnO}_{3}$ [229], $\mathrm{LuMnO}_{3}$ [230], and $\mathrm{BiFeO}_{3}[73,231-235]$. Room temperature exchange coupling effects have been obtained with $\mathrm{BiFeO}_{3}$, which exhibits a Néel temperature of $643 \mathrm{~K}$. The origin of exchange bias in ferromagnetic films on $\mathrm{BiFeO}_{3}$ depends on the type of ferroelectric domain walls. If the domains are predominantly separated by $109^{\circ}$ walls, the exchange bias field is inversely proportional to the ferroelectric domain size $[231,232]$. For $71^{\circ}$ walls, no shift in the hysteresis loop is measured [231, 235]. In this case, exchange interactions between the ferromagnetic film and $\mathrm{BiFeO}_{3}$ result in an enhancement of the coercive field, which is explained by direct coupling to the canted moment of $\mathrm{BiFeO}_{3}$ domains. The orientation of the canted moment in $\mathrm{BiFeO}_{3}$ is strongly linked to the direction of ferroelectric polarization. Consequently, rotation of the polarization produces a lateral modulation of magnetic anisotropy in the ferromagnetic film. This effect can lead to ferroelectric/ferromagnetic domain correlations, which form a strong basis for electric-field controlled magnetic switching in exchange-coupled systems [73, 233, 235]. A detailed review of electric-field control of magnetism using $\mathrm{BiFeO}_{3}-$ based heterostructures can be found elsewhere [236]. An overview of popular inorganic multiferroic composites for electric-field control of magnetism and their properties is given in Table III.

Table III. Overview of popular multiferroic composite systems, their properties, and functions.

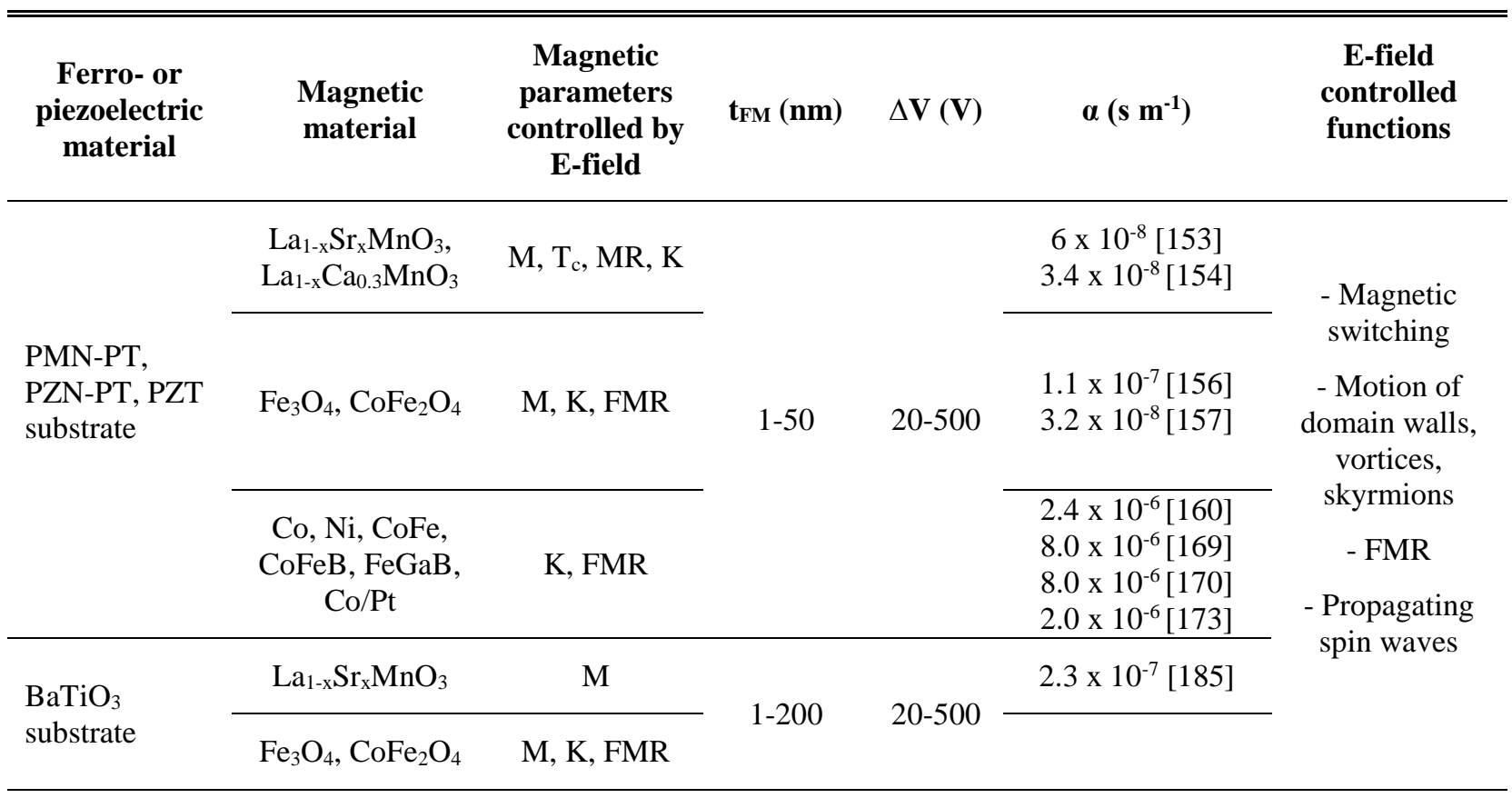




\begin{tabular}{|c|c|c|c|c|c|}
\hline $\begin{array}{l}\mathrm{Fe}, \mathrm{Ni}, \mathrm{CoFe} \\
\mathrm{CoFeB}, \mathrm{FeGa}\end{array}$ & K, FMR & & & $6.0 \times 10^{-7}[192]$ & \\
\hline $\mathrm{FeRh}$ & M & & & $1.4 \times 10^{-6}[196]$ & \\
\hline $\begin{array}{l}\mathrm{La}_{1-\mathrm{x}} \mathrm{Sr}_{\mathrm{x}} \mathrm{MnO}_{3} \\
\mathrm{La}_{1-\mathrm{x}} \mathrm{Ca}_{0.3} \mathrm{MnO}_{3}\end{array}$ & $\mathrm{M}, \mathrm{T}_{\mathrm{c}}, \mathrm{MR}, \mathrm{K}$ & $<10$ & \multirow{2}{*}{$1-10$} & $\begin{array}{l}8.0 \times 10^{-10}[215] \\
6.2 \times 10^{-9}[216]\end{array}$ & \multirow{2}{*}{$\begin{array}{c}\text { - Magnetic } \\
\text { switching } \\
\text { - Spin } \\
\text { polarization }\end{array}$} \\
\hline $\mathrm{Fe}, \mathrm{Co}$ & $\mathrm{M}, \mathrm{K}, \mathrm{SP}$ & $<3(\mathrm{M}, \mathrm{K})$ & & & \\
\hline $\mathrm{La}_{1-\mathrm{x}} \mathrm{Sr}_{\mathrm{x}} \mathrm{MnO}_{3}$ & $\mathrm{~K}, \mathrm{H}_{\mathrm{ex}}$ & \multirow{2}{*}{$<10$} & \multirow{2}{*}{$1-10$} & & \multirow{2}{*}{$\begin{array}{c}\text { - Magnetic } \\
\text { switching } \\
\text { - FMR }\end{array}$} \\
\hline $\mathrm{CoFe}$ & $\mathrm{K}, \mathrm{FMR}, \mathrm{H}_{\mathrm{ex}}$ & & & $1.0 \times 10^{-7}[67]$ & \\
\hline
\end{tabular}

Electric-field control of magnetic switching, magnetic domain-wall or skyrmion motion, and magnetization dynamics are relevant for energy-efficient magnetic memories, logic devices, and tunable microwave components. Magnetic switching requires a change of magnetic anisotropy during voltage pulsing. In strain-coupled multiferroic composites, reversible $90^{\circ}$ switching between two in-plane states or in-plane and perpendicular magnetization has been demonstrated for various material combinations. Because elastic energy is an even function of magnetization, technologically more attractive $180^{\circ}$ switching requires an additional symmetrybreaking magnetic energy. For instance, the concurrent application of magnetic field pulses has been shown to trigger $180^{\circ}$ in-plane switching in ferromagnetic films on PMN-PT [174, 237] and full perpendicular magnetization reversal in $\mathrm{Cu} / \mathrm{Ni}$ multilayers on $\mathrm{BaTiO}_{3}$ [199]. Other strategies involve the use of competing shape anisotropies [238-240], dipolar coupling to a nanomagnet [241], multiple electrodes [242, 243], strain-pulse-induced magnetization precession [181-183], and spin-orbit torques [244]. All-electric $180^{\circ}$ magnetic switching has been demonstrated in an exchange-coupled $\mathrm{Co}_{0.9} \mathrm{Fe}_{0.1} / \mathrm{BiFeO}_{3}$ bilayer [74]. Two-step ferroelectric switching in the $\mathrm{BiFeO}_{3}$ film, realized through strain-engineered growth onto a $\mathrm{DySCO}_{3}(110)$ substrate, breaks the timereversal symmetry in this thin-film heterostructure. In recent years, magnetic switching based on exchange coupling between a single-phase multiferroic film and a magnetic spin-valve has been developed for ME spin-orbit logic [75].

ME control over the motion of magnetic domain walls has been studied in various composite multiferroic heterostructures. For instance, strain-mediated changes in magnetic anisotropy have been shown to alter the pinning strength and motion of magnetic-field or current-driven magnetic domain walls in Co/Cu/CoFeB and GaMnAsP on top of PZT [245, 246]. Pure electricfield-driven reversible magnetic domain wall motion has been realized in $\mathrm{Fe} / \mathrm{BaTiO}_{3}$ and $[\mathrm{Cu} / \mathrm{Ni}]_{\mathrm{N}} / \mathrm{Cu} / \mathrm{BaTiO}_{3}$ by pinning magnetic domain walls onto mobile ferroelectric domain boundaries [247, 248]. Further reports on strain-mediated effects include electric-field controlled motion of magnetic domain walls around the circumference of magnetic ring structures on PMNPT $[249,250]$ and electric-field tuning of the chirality of magnetic vortex walls in Ni/PMN-PT [159]. Other non-collinear spin structures such as vortices have been manipulated by electric fields in $\mathrm{Ni}$ nanodisks on PMN-PT $[252,253]$. Particularly interesting is the prospect of electric-field control of topologically protected magnetic skyrmions which, because of their small size and 
stability, are a promising candidate as information carrier in future spintronics devices. Micromagnetic simulations indicate that strain transfer between a piezoelectric layer and a thin ferromagnetic film with perpendicular magnetic anisotropy and interfacial Dzyaloshinskii-Moriya interaction facilitates electric-field-controlled nucleation and motion of magnetic skyrmions [254, 255]. Experimental demonstrations of these phenomena are expected in the foreseeable future.

Tuning of magnetization dynamics in ME composites focused initially on uniform FMR excitations. Giant shifts of the FMR frequency by fields up to 750 Oe or several GHz have been demonstrated in several inorganic strain-coupled bilayers, including FeGaB/PZN-PT [256], $\mathrm{Fe}_{3} \mathrm{O}_{4} / \mathrm{PZN}-\mathrm{PT}$ [163], and CoFeB/PMN-PT [174]. Electric-field manipulation of FMR has been achieved also through exchange coupling in $\mathrm{CoFeB} / \mathrm{BiFeO}_{3}$, but with smaller variations in the resonance field [257]. Lowpower manipulation of propagating spin waves by electric fields is of interest to ultrafast beyondCMOS parallel computing [258]. All-electrical emission of propagating spin waves by local ac-field actuation of a PMN-PT substrate with a patterned Ni/NiFe waveguide on top has been reported in Ref. 259. Other magnonic functionalities exploiting local piezostrain transfer include active manipulation of spin-wave transport in a ferromagnetic metal film with a PMN-PT gate [260] and reconfigurable spin-wave routing in coupled yttrium iron garnet stripes underneath a PZT layer [261]. Coupling of a magnetic film to the ferroelastic domains of $\mathrm{BaTiO}_{3}$ opens additional opportunities. Pinned magnetic domain walls and magnetic anisotropy boundaries in this multiferroic composite can be used as tunable source of short-wavelength spin waves [262, 263], while switching of the magnetic domain wall structure allows spin-wave signals to be turned on and off reversibly [264].

\section{Future trends in inorganic multiferroic composites}

Inorganic multiferroic composites operating at room temperature and above have been exploited successfully in various ME devices, including mechanical antennas, magnetic field and current sensors, voltage tunable inductors and filters, energy harvesters, isolators, and gyrators (see Section 3). The ability to tailor the ME coupling strength through material selection, interface engineering, or changes in phase connectivity provides flexibility in the design of ME systems utilizing magnetic-field-to-voltage conversions or electric-field tunable magnetic effects. Information technology is another potential application area of multiferroic composites. Memory and logic devices both require fast operation on the nanoscale, which poses a future challenge for magnetoelectrics. While several device concepts have been proposed during the last decade, the first realization of ME spin-orbit logic was reported by Intel only recently [68]. Further advances in this direction are expected in the coming years. The integration of ME components in scalable information technology requires further material and nano-structuring development. For instance, electric-field controlled multiferroic composites need to be scaled down to limit the device footprint and operation voltage. This is particularly arduous for thin-film systems relying on strain transfer, as substrate clamping limits the ME coupling strength. Patterning of both ferroic phases may mitigate this restriction. Downscaling of the piezo- or ferroelectric layer thickness raises additional questions about the engineering of suitable domain patterns, leakage, and issues related to voltage breakdown and fatigue. Experimental techniques revealing the atomic-scale mechanisms underlying ME coupling in multiferroic composites, such as in situ transmission electron microscopy, could provide valuable insights on how to improve the 
performance and endurance of inorganic multiferroic nanocomposites. Material and processing compatibility with industrial fabrication standards is another important requirement for the realization of ME-based information technology. The development of lead-free room temperature piezo- and ferroelectric thin films and the growth of epitaxial ME composites onto silicon will aid the ongoing efforts towards sustainable device fabrication.

\subsection{Multiferroic magnetoelectric polymers}

The ME effect, proposed by Pierre Curie in 1894 [1], establishes a relation between magnetic and electric properties of matter, promoting the magnetically induced variation of electrical polarization and/or the electrically induced variation of the magnetization. Thereafter in 1960, Dzyaloshinskii [265] (by symmetry considerations) envisioned this effect that was subsequently experimentally proven by Astrov [2,266]. In 1966, Ascher and Schmidt (Battelle Institute, Geneva, Switzerland) and Newnham (Pennsylvania State University, Philadelphia, USA) discovered a high number of boracites [267] and phosphates [268] that exhibited important ME properties. After two decades of intense experimental and theoretical work, the state of the art and research on such single-phase MEs reached a saturation, mainly based on the restricted number of compounds displaying it, the low-temperature needed for the coupling to occur, the difficulties in developing useful applications, and by the limited understanding of the microscopic sources of the ME behaviour [13].

At the beginning of the new millennium the main issues of single-phase ME materials have been solved by the development of two-phase composite multiferroics, that consist on a piezoelectric phase and a magnetostrictive phase [16]. In such composites, the ME effect arises from the interaction of the elastic components of the piezoelectric and magnetostrictive constituents: i) an applied electric field induces strain in the piezoelectric phase; ii) such strain is transmitted to the magnetostrictive phase; iii) the strain leads to magnetic variations in the magnetostrictive material. Thus, the ME effect is high if the coupling at the interface is effective; consequently, composites with large surface area (such as multi-layered films or nanocomposites) and strongly ferroelastic elements are particularly being implemented [12]. The reported ME coefficients defined as [16]:

$$
\alpha_{i j}=\frac{\Delta V}{t \cdot H_{A C}}
$$

where $\Delta V$ is the voltage generated on the piezoelectric material, $t$ is the thickness of the piezoelectric material, $H_{A C}$ the magnitude of the applied $A C$ magnetic field and $i$ and $j$ the direction of the voltage measurement and applied $H_{A C}$, respectively, were three orders of magnitude higher than the ones observed in single-phase ME materials (some $\mathrm{mV} . \mathrm{cm}^{-1} \cdot \mathrm{Oe}^{-1}$ ).

In this context, polymer-based ME composites are particularly interesting due to their simple processability, flexibility, moldability, high electrical resistivity, improved mechanical properties 
and suitability for implementation in the Industry 4.0 and the Internet of Things (IoT) paradigms [269-273].

\section{Materials}

Two main types of ME polymer-based composites have been mainly developed: nanocomposites and laminated composites [270]. In (0-3) particulated nanocomposites, a high concentration of magnetic particles is dispersed into the selected piezopolymer matrix [274, 275]. The characteristics of the resulting composite can be optimized by selecting the phase types, the nanoparticle size, and the processing parameters. Otherwise, in (2-2) laminated composites, the piezoelectric and magnetostrictive components are typically linked with a coupling agent such as an epoxy and results on a larger ME performance ( 3 orders of magnitude higher than in nanocomposites) [276-278]. On the other hand, these laminated composites may have potential segregation and leakage current problems [269,271]. Such laminated composited can be arranged in different forms and geometries, including discs, squares, rectangles, and rings, with different sizes [279, 280].

Figure 5 summarizes the most used materials for the development of ME polymer-based composites.
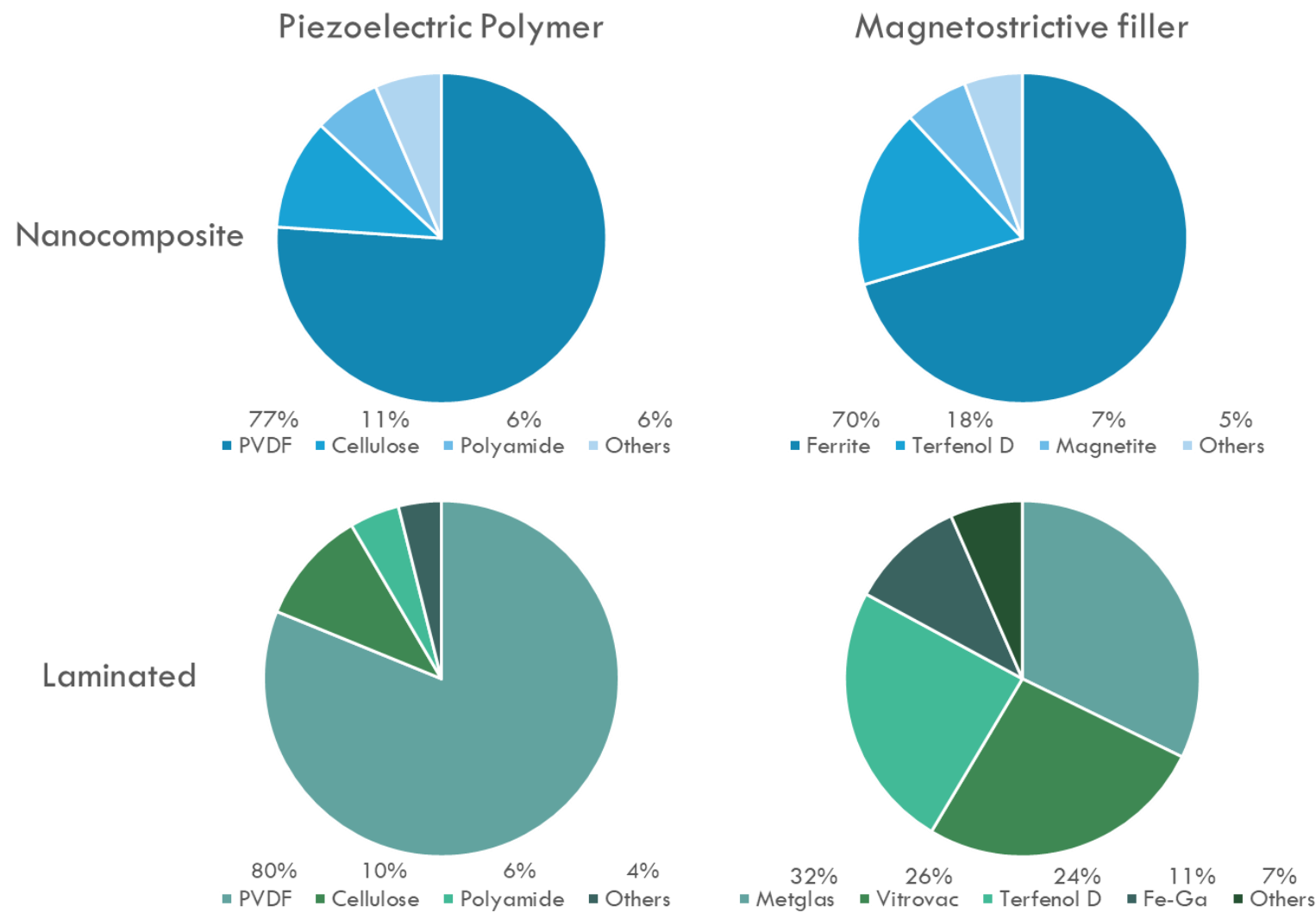

Fig. 5. Most used piezoelectric and magnetostrictive materials for the development of polymerbased ME composites: nanocomposites and laminated composites. Source: SCOPUS database (accessed on 25/11/2020). 
Due to their highest dielectric constant and electroactive response, including piezoelectric $\left(\left|d_{33}\right| \approx 30\right.$ pc. $\left.N^{-1}\right)$ and ferroelectric effects [281], poly(vinylidene fluoride), PVDF, and its copolymers are the polymers preferentially used both in nanocomposites (representing $\approx 77 \%$ of the total) and laminated composites (representing $\approx 80 \%$ of the total). Concerning the magnetostrictive element, ferrites such as $\mathrm{CoFe}_{2} \mathrm{O}_{4}, \mathrm{Ni}_{0.5} \mathrm{Zn}_{0.5} \mathrm{Fe}_{2} \mathrm{O}_{4}$ or $\mathrm{NiFe}_{2} \mathrm{O}_{4}$ are responsible for $\approx 70 \%$ of the studies regarding nanocomposites and Metglas appears on $\approx 32 \%$ of the laminatedbased reports. Those facts are explained by the tailorable magnetic hysteresis, coercivity and high magnetostrictive coefficients (up to some dozens of ppms) of ferrites $[280,282]$ and by the high magnetic permeability, piezomagnetic coefficient $\left(2 \mathrm{ppm} . \mathrm{Oe}^{-1}\right)$ and stiffness of Metglas [283, 284].

Such materials have offered optimized features that triggered new applications with easy production at low temperatures and additive manufacturing capability $[285,286]$ (inkjet printing, screen printing, and spray-printing, among others), adjusted mechanical properties for flexible devices, large area devices, and biocompatible applications [279]. This will be discussed in the next chapter.

As mentioned in the introduction, one decade ago polymer-based ME materials emerged as a possible solution to solve the main problems related to single-phase multiferroic materials and quickly revealed strong potential applicability in the areas of sensors, actuators, energy harvesting, memories and biomedical materials.
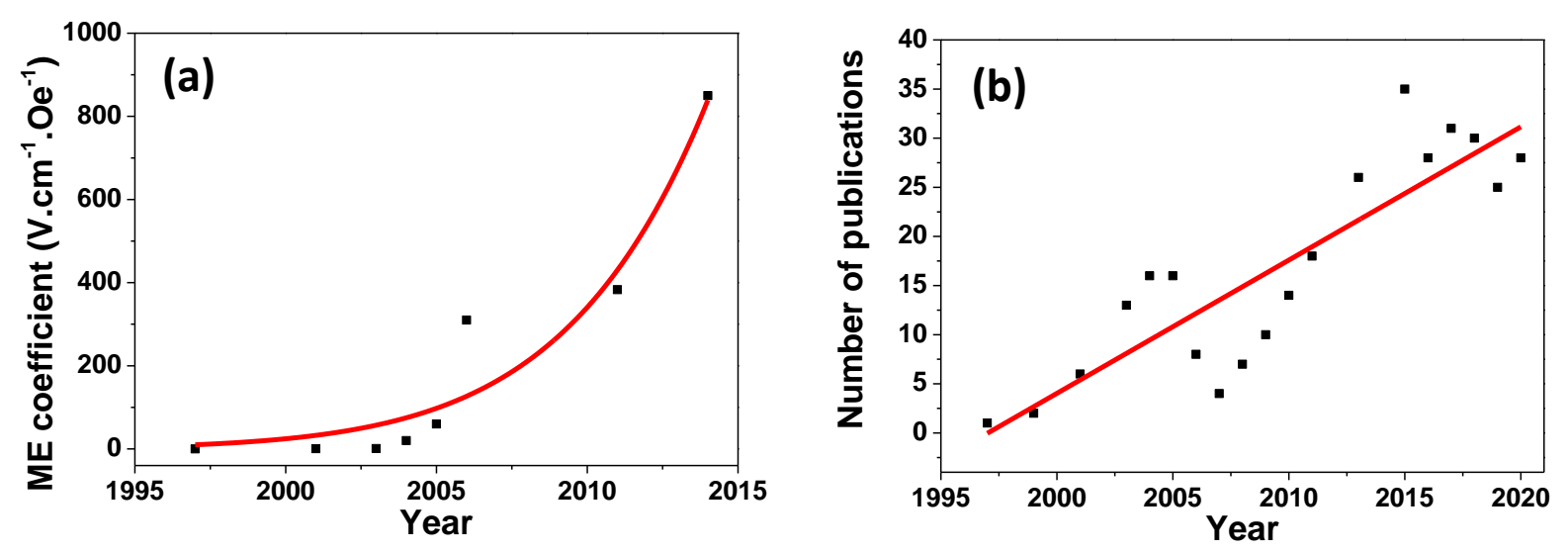

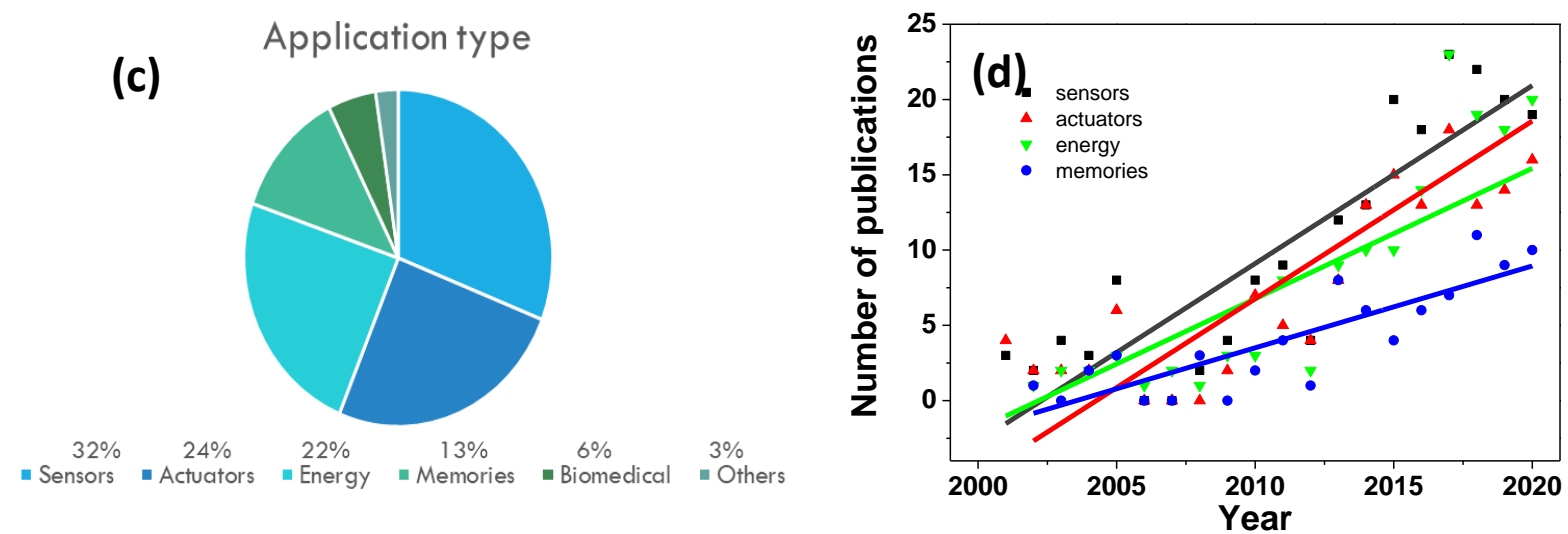

Fig. 6. Maximum ME coefficient in ME polymer composites over time (a) and the number of publications (b) on polymer-based ME materials along the years. Distribution of the applicationoriented papers among the different applications (c) and as a function of the publishing year. Source: SCOPUS database (accessed on 25/11/2020).

While ME coefficients up to some $\mathrm{mV} \cdot \mathrm{cm}^{-1} \cdot \mathrm{Oe}^{-1}$ were reported on single-phase ME materials in the last decade of the previous millennium, work on polymer-based ME materials increased this value by almost 1 million times (see Fig. 6(a)), being reported the highest ME coupling (850 V.cm ${ }^{1} . \mathrm{Oe}^{-1}$ ) in 2014 for a polyvinylidenefluoride-co-trifluoroethylene-P(VDF-TrFE)/Metglas laminate [287]. Such highest ME response obtained on polymer-based materials was reported for the area of magnetic sensing, whereas for energy harvesting, the highest ME coefficient that has been implemented has been $250 \mathrm{~V} \mathrm{~cm}^{-1} \mathrm{Oe}^{-1}$ [283]. Magnetic sensors are in fact the most popular application of polymer-based ME materials, representing $30 \%$ of all presented applications (Fig. 6(b)). ME polymeric composites developed for data memories, actuators and biomedical engineering exhibit an intermediate ME coupling [270].

When compared to alternative technologies, polymer-based ME materials display: i) large 15 $\mathrm{kV} . \mathrm{T}^{-1}$ sensitivity as a result of the enhanced magnetic flux concentration effect [288] (a sensitivity of $0.005 \mathrm{kV} . \mathrm{T}^{-1}$ has been reported for Hall sensing devices [289]); ii) a $1 \mathrm{~mm} / \mathrm{T}$ displacement actuation capability [290] (0.03 value has been reported for single crystal-based actuators [291]);iii) a power output (power/volume/HAC of $3.3 \mathrm{~mW} \cdot \mathrm{cm}^{-3} \cdot \mathrm{Oe}^{-1}$ [292] $\left(3 \mathrm{~mW} \cdot \mathrm{cm}^{-3} \cdot \mathrm{Oe}^{-1}\right.$ has been the value reported on helical core magnetic harvesters [293]); and iv) 4 memory states ( 2 can be found on traditional magnetic recording devices [294]). Regarding biomedical applications, polymer-based ME composites have attracted the attention for tissue engineering, drug delivery, surgical actuation, neural simulation, biomedical sensing, antennas for biomedical implants and energy harvesters for small biomedical devices [295-298] due to their biocompatibility and sensing/actuation/harvesting performances [270, 279, 297].

The increasing number of publications (Fig. 6(b)) and application-oriented publications (Fig. 6(d)) indicate that in the coming years new applications and application-trends should appear.

\section{Future trends in Multiferroic magnetoelectric polymers}


In the future, the quest for increased ME coefficients as observed from 1995 to 2015 is expected to slow down once the voltage output of the reported materials is already enough for most of the applications explored and presented in in this article, being the only exception and for the obvious reasons, the ME energy harvesters, demanding substantial higher coupling.

Due to environmental reasons and to the European green deal, the rational design of higher performance and environmental friendlier polymer-based ME materials, having high ME coupling, low leakage currents, and high remanent magnetization/coercivity should have high priority.

The exploration of new ME couplings resulting from magnetic skyrmions, polar vortices, ionic composites, among others, could open new application avenues such as ME gels, magnetic batteries, radio frequency switches, resonators, magnetoactive flexible displays, soft haptic ME devices and magnetically controlled millimetre-scale robotics.

Cross effects with additional stimulus (optical, acoustic and thermal) will enable monitoring and real time optimization and autonomy associated with Internet of Things (IoT) and industry 4.0, by the introduction of self-powered sensors and actuators for on-time sensing, monitoring and response, enabling at the same time energy harvesting from the surrounding environments.

Regarding the development of modern magnetoactive electronics, ensuring the magnetic, electrical and thermal stability at the $10-\mathrm{nm}$ length scale is one of the most important requirements that will rely on advanced characterization tools in order to study the ME coupling at the nanometer and atomic length-scales. The successful implementation of ME devices will be strongly related with the joint vision/work between electronics (signal processing and integration), processing techniques (additive manufacturing and scale-up) and materials engineering (magnetostrictive, piezoelectric and mechanical optimization).

In the last 20 years extensive progress has been achieved in the area of ME materials based on different kinds of ME structures. Such optimized performance was greatly benefited from the optimized piezomagnetic/magnetostrictive coefficients of the magnetostrictive materials, which lead to a strong ME coupling. From a scientific point of view, little has been achieved on the optimization of the piezoelectric response of the polymer matrices: novel approaches to improve the piezoelectric properties and interface coupling with the magnetic component of polymerbased ME materials is thus one of the biggest challenge in the field.

With all this scenario it is highly likely that no substantial increase of the ME coefficient should be reported in the coming years, otherwise the "so far linear" increase observed in the number of publications and applications regarding ME materials should follow one of two paths: i) stagnate, if the electronic, processing, materials engineering and industries "worlds" do not synergistically reinforce each other with novel materials, applications and technologies; ii) further increase if the requirements of additive manufacturing, precise integration and adaptation to the paradigms of Green Deal, loT and 4.0 revolution are timely fulfilled, staring tomorrow....

\subsection{Surface-charged and magneto-ionic materials}

Besides single-phase and heterostructured multiferroic materials, other strategies have emerged during the last 25 years to tailor the magnetic properties of materials without the use of piezoelectricity or ferroelectricity. Indeed, more than two decades ago, various studies showed 
the possibility to control the magnetic properties of diluted magnetic semiconductors at low temperature directly with voltage, without strain-coupling effects. Accumulated electric charges can induce changes in the carrier density of these semiconductors (donors or holes) and, as a result, the exchange interactions responsible for ferromagnetism become modified [299, 300]. Changes in Curie temperature $\left(T_{C}\right)$, coercivity $\left(H_{c}\right)$, saturation magnetization $\left(M_{S}\right)$ or magnetic easy axis were reported in these materials under the action of electric field. Some years later, in 2007, Weisheit et al. demonstrated that voltage could also be used to tune the coercivity of ultrathin magnetic metallic films [301-303]. The influence of electric charging on the magnetic properties of thin films, multilayers and nanostructures has been extensively reviewed from a theoretical viewpoint by Brovko et al. [304]. Song et al. have reviewed the general mechanisms responsible for voltage control of magnetism [7]. In turn, Leistner et al. [305], Molinari et al. [8] and Navarro-Senent et al. [9], has also revised the main results concerning electrolyte-gating of magnetic materials. Since electric field in metals is effectively screened at the surface (within the Thomas-Fermi length, which is about $0.5 \mathrm{~nm}$ ), such effects required of film thicknesses of the order of 2-3 nm. Not only electrostatic charges can alter the wave function and the occupancy of the $d$ orbitals of metallic alloys, but electric field screening depends also on the orientation of the spin. Thus, such electric charging can indeed be an effective procedure to tune anisotropy and coercivity in semiconductors and metals, eventually reducing the energy required for the writing of magnetic information. Remarkably, although the most drastic effects occur at the very outer surface of the alloy, the induced magnetic changes can propagate within the exchange correlation length, which can be up to $20 \mathrm{~nm}$. Inspired by these seminal works on electric surface charging, several works have reported lately on the possibility to tailor the magnetic properties of nanoporous alloys directly with voltage, taking advantages of their high surface area-tovolume ratio [9]. Drastic effects have been demonstrated in relatively thick (few hundred $\mathrm{nm}$ ) nanoporous metallic films (e.g., Cu-Ni [299], Co-Pt [307], Fe-Cu [308]) with ultra-narrow pore walls $(\sim 5 \mathrm{~nm})$, prepared by electrodeposition procedures using suitable surfactants or colloidal templated substrates. This way of modifying magnetism can overcome some of the problems of heterostructured multiferroics, such as the limited available strains when ferroelectric/ferromagnetic bilayers are deposited onto rigid substrates or the need of epitaxy and perfect interfaces for efficient transfer of strain. Note, however, that this procedure (electric surface charging) only allows for converse ME effects, not direct, since it does not allow generating electricity using alternating magnetic fields.

Besides strain and electric surface charging, applied electric fields can also induce changes in the oxidation state of magnetic metallic alloys or semiconductors (i.e., reduction/oxidation reactions or ion migration), particularly when they are immersed in liquid electrolytes or grown adjacent to ionically conducting buffer layers (solid electrolytes) [8, 9, 309-320]. Electric-field-induced oxygen motion in magnetic materials has recently revolutionized voltage control of magnetism since this mechanism may allow for an unprecedented modulation of magnetic properties $\left(\mathrm{M}_{\mathrm{s}}\right.$, $\mathrm{H}_{\mathrm{C}}$ ) in either permanent or temporary ways (if effects are reversible upon application of voltage of opposite polarity) [311, 314, 318, 321-323]. Various terminologies have been used to designate magnetic effects arising from voltage-driven ion migration in magnetic materials, such as electrochemical, ion exchange, redox or magneto-ionic effects, although the latter term had been traditionally used to describe the propagation of electromagnetic waves by an ionized medium 
under an external magnetic field (e.g., combined effect of the Earth's magnetic field and atmospheric ionization). Magneto-ionics has been carried out by immersing metallic films and nanoporous metallic alloys in aqueous electrolytes [309,324,325]. Subsequent works also showed the possibility to modify the magnetic properties of metal/metal oxide nanocomposites $[310,326]$ or transition metal oxide materials (e.g., $\mathrm{Fe}_{2} \mathrm{O}_{3}, \mathrm{Co}_{3} \mathrm{O}_{4}$ ) with voltage when immersing them either in aqueous [312] or aprotic polar electrolytes [311, 318, 327-329], in all cases assisted by redox reactions or $\mathrm{O}^{2-}$ ion motion. A schematic drawing, depicting magneto-ionic effects in FeOx nanoporous layers, when immersed in a polar electrolyte, is shown in Fig. 7. The properties of FM or ferrimagnetic oxides have also been tuned by voltage-driven lithiation ( $\mathrm{Li}^{+}$incorporation using suitable electrolytes) [330-333]. Recent works have also reported magneto-ionic effects arising from fluor [334] or nitrogen [319] ion migration. Nitrogen magneto-ionics offers some advantages with respect to oxygen magneto-ionics in terms of operation speed, threshold voltages to induce the ME effects and improved cyclability [319].

Magneto-ionics can be relevant beyond the outer surface of alloys, hence extending throughout the bulk of materials [315]. Magneto-ionics envisages writing energies in magnetic bits of the order of $10^{-3} \mathrm{fJ} / \mathrm{bit}=1 \mathrm{aJ} / \mathrm{bit}[314,335]$, which is two orders of magnitude lower than those required in complementary metal oxide semiconductor (CMOS) technology $\left(\sim 10^{-1} \mathrm{fJ} / \mathrm{bit}\right)$. In solid state, magneto-ionic systems usually comprise multilayer heterostructures in a capacitor-like configuration. Ferromagnetic metals, such as $\mathrm{Co}$ or Fe, are the target materials grown adjacent to solid electrolytes, such as $\mathrm{GdO}_{x}$ or $\mathrm{HfO}_{2}$, which act as ion reservoirs, accepting or donating oxygen ions depending on the gating polarity [317]. Magneto-ionic effects have been also observed in nanoporous composites comprising a magnetic porous framework with the pore walls coated by ionic conductors (e.g., $\mathrm{HfO}_{x}$ ) by atomic layer deposition [336,337]. Importantly, one of the drawbacks of electrochemical processes is that the effects are not induced instantaneously (until today, the dynamics is relatively slow, particularly at room temperature, where times of the order of several seconds or minutes are typically required). This is hampering the utilization of magneto-ionics in high-speed memory applications and spintronics, although some authors argue the so-far attained switching speeds could be suitable for neuromorphic computing. The slow dynamics is due to the pronounced structural and compositional changes that the pristine ferromagnetic layers suffer during voltage application, also leading to irreversibility and, often, limited cyclability.

Recently, via a proton-based (hydrogen ions) approach, better endurance and $10^{-3} \mathrm{~s}$ room temperature operation has been shown feasible [321]. Electrochemical hydrogenation of metallic alloys takes place via voltage-controlled reduction of $\mathrm{H}^{+}$to atomic $\mathrm{H}$ and subsequent $\mathrm{H}$ absorption at the surface of the metal. Hydrogen atoms can diffuse via interstitial sites at high mobility rates (due to their small size), rendering hydrogen-mediated magneto-ionics a very promising approach to circumvent the limited operation speed and cyclability of oxygen magneto-ionics. Hydrogen magneto-ionics have been demonstrated both in solid and liquid [305, 320, 321, 338344] electrolyte configurations. In solid state, Tan et al. [321] showed a voltage-induced spin reorientation in $\mathrm{Au} / \mathrm{GdO}_{\mathrm{x}} / \mathrm{Co}(0.9 \mathrm{~nm}) / \mathrm{Pt}$ stacks assisted by humid environment, with endurance of up to 2000 cycles, wherein hydrogen accumulation at the $\mathrm{GdO}_{\mathrm{x}} / \mathrm{Co}$ interface caused a modification of the interface magnetic anisotropy. Using liquid aqueous electrolyte configuration, 
Maroun et al. demonstrated a reversible increase and decrease of perpendicular coercivity in $\mathrm{Pd} / \mathrm{Co} / \mathrm{Au}$ multilayer stacks arising from hydrogenation of the Pd layer [338]. A transition from superparamagnetic to ferromagnetic behavior [18] was demonstrated in nanoporous $\mathrm{Pd}(\mathrm{Co})$ assisted by a Ruderman-Kittel-Kasuya-Yosida-type interaction in the Pd matrix, also due to electrochemical $\mathrm{H}^{+}$reduction to $\mathrm{H}$ and subsequent $\mathrm{H}$ absorption in this material [342]. Hydrogenbased magneto-ionics was also shown in $\mathrm{SrCoO}_{3-\delta}$ [343] and $\mathrm{NiCO}_{2} \mathrm{O}_{4}$ films [344]. Remarkably, hydrogen insertion using aqueous electrolytes has been achieved in $\mathrm{SmCO}_{5}$ and $\mathrm{Sm}_{2} \mathrm{Co}_{17}$ hard magnets, where changes in $\mathrm{H}_{c}$ above $1 \mathrm{~T}$ have been reported $[339,340]$. Finally, hydrogen exposure of ultrathin Fe films has been recently reported to be a suitable strategy to induce skyrmions in this material [341].

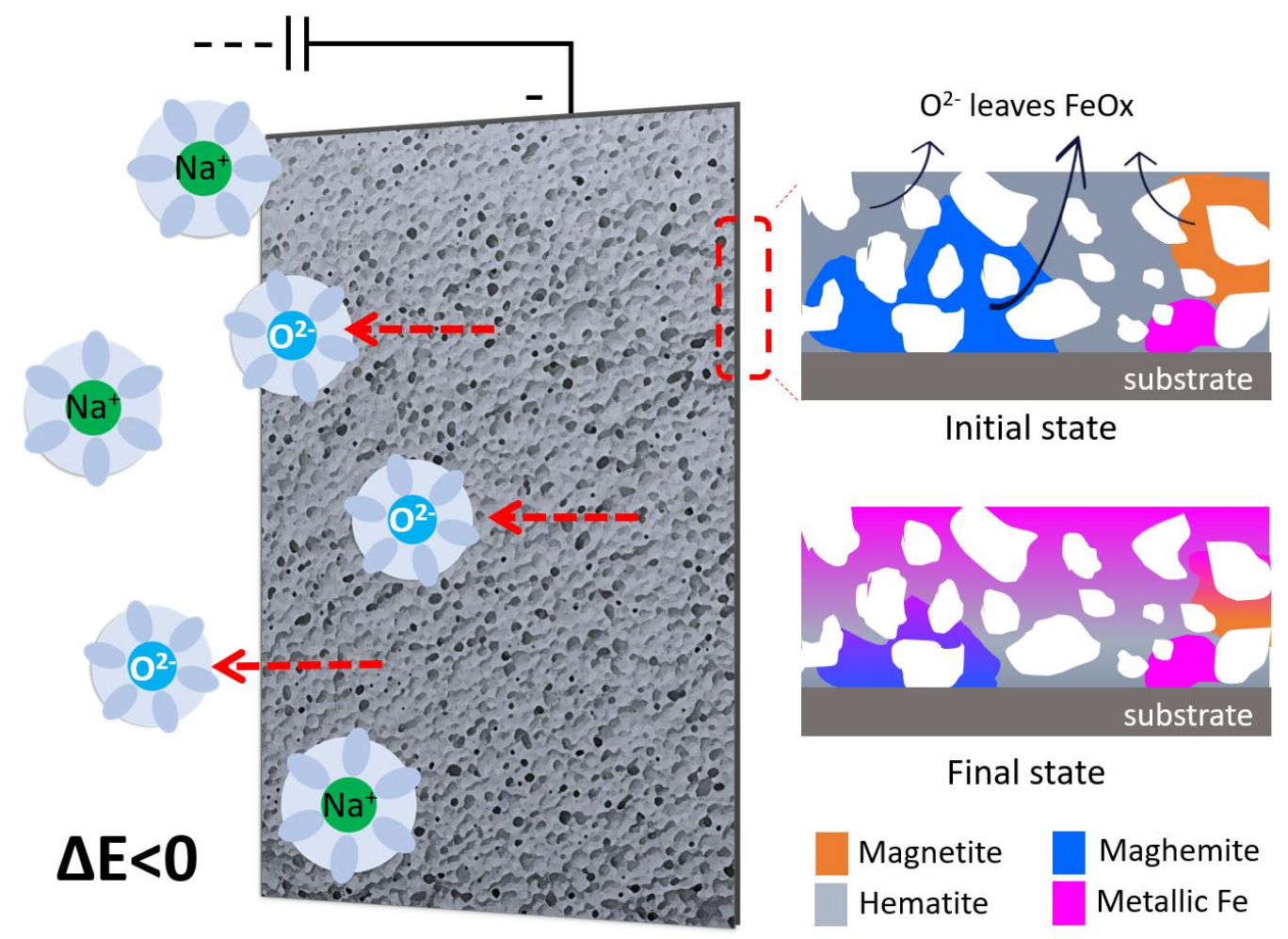

Fig. 7. Illustration of magneto-ionic effect in the porous $\mathrm{FeO}_{x}$ films subject to negative voltage polarity. Adapted from Ref. 327.

\section{Future trends in surface-charged and magneto-ionic materials}

Table IV reports on the recent progress achieved in tuning of $\mathrm{M}_{\mathrm{s}}$ and $\mathrm{H}_{\mathrm{C}}$ using magneto-ionic phenomena in a large variety of metallic alloys and oxide materials. In spite of the recent progress, there are still some challenges to be faced in the future, both for surface charging and magnetoionics. Voltage-driven modulation in magnetic semiconductors is still rather limited to low temperatures mainly due to the scarcity of room-temperature magnetic semiconductors. Efficient surface charging in metals is limited to very thin films and nanoporous alloys. However, 
integration of porous alloys in spintronics is not straightforward (from the viewpoint of existing synthetic procedures) and it is detrimental in the current trends towards device miniaturization. Moreover, most studies are still performed in liquid electrolytes and extrapolation to all-solid configurations is sometimes difficult due to the occurrence of electric pinholes in the solid dielectric layers used to generate the electric field. The use of advanced atomic layer deposition techniques could overcome some of these problems. Concerning magneto-ionics, the currently available operation speeds are still too slow compared to other types of memories and existing materials often suffer from relatively poor endurance, where cyclability larger than $10^{3}$ is rarely reported. The use of proton intercalation and other types of small ions can circumvent some of these issues, although there are still problems related to long-term ion retentivity. Structural defects-engineering to create large amounts of grain boundaries, along which ionic diffusion preferentially occurs, is another interesting approach to accelerate magneto-ionic rates. In this sense, combining ion irradiation or ion implantation with voltage-actuation could be a suitable strategy to boost magneto-ionics in the future.

Table IV: Representative systems and changes in $\mathrm{M}$ and $\mathrm{H}_{\mathrm{C}}$ obtained using magneto-ionic effects. Also indicated are the types of electrolytes needed and the eventual reversibility. Adapted from Ref. 9.

\begin{tabular}{|c|c|c|c|c|c|}
\hline Material & Electrolyte & $\begin{array}{l}\text { Magnetic } \\
\text { parameter }\end{array}$ & Variation & Reversibility & Ref. \\
\hline \multirow{2}{*}{$\begin{array}{c}\mathrm{Co} / \mathrm{Ni} / \mathrm{HfO}_{2} \text { films } \\
{[\mathrm{Co} / \mathrm{Ni}]_{3} / \mathrm{HfO}_{2} \text { films }}\end{array}$} & \multirow{2}{*}{ DEME-TFSI } & $\mathrm{M}_{\mathrm{S}}$ & $\Delta \mathrm{M}_{\mathrm{S}} \approx+70 \%$ & Yes ( 10 min) & \multirow{2}{*}[345]{} \\
\hline & & $\mathrm{H}_{\mathrm{C}}$ & $\Delta \mathrm{H}_{\mathrm{C}} \approx 74 \%$ & Partially ( $30 \mathrm{~min})$ & \\
\hline Co ultra-thin film & MaN2401 & $\mathrm{H}_{\mathrm{C}}$ & $\Delta \mathrm{H}_{\mathrm{C}} \sim 68 \%$ & Yes ( 30 min) & [346] \\
\hline $\begin{array}{l}\mathrm{SrCoO}_{3-\delta}, \mathrm{SrCoO}_{2.5} \\
\text { and } \mathrm{HSrCoO}_{2.5} \text { films }\end{array}$ & DEME-TFSI & $\begin{array}{l}\text { Phase } \\
\text { transition }\end{array}$ & $\begin{array}{c}\text { FM insulator-FM } \\
\text { metal-AFM } \\
\text { insulator phase } \\
\text { transition }\end{array}$ & Yes $(\sim \min )$ & {$[343]$} \\
\hline Fe-Pt ultra-thin films & $\begin{array}{l}\mathrm{LiPF}_{6} \text { in } \\
\mathrm{DMC} / \mathrm{EC}\end{array}$ & $\mathrm{M}_{\mathrm{S}}$ & $\Delta \mathrm{M}_{\mathrm{S}} \approx 8 \%$ & Yes & {$[347]$} \\
\hline $\begin{array}{l}\text { FePt/iron oxide } \\
\text { composites }\end{array}$ & $\begin{array}{l}\mathrm{LiPF}_{6} \text { in } \\
\mathrm{DMC} / \mathrm{EC}\end{array}$ & $\mathrm{M}_{\mathrm{S}}$ & $\Delta \mathrm{M}_{\mathrm{S}} \approx+4 \%$ & Yes & [348] \\
\hline$\gamma-\mathrm{Fe}_{2} \mathrm{O}_{3}$ nanoparticles & $\begin{array}{l}\mathrm{LiPF}_{6} \text { in } \\
\mathrm{EC} / \mathrm{DEC}\end{array}$ & $\mathrm{m}$ & $\Delta \mathrm{m} \approx 20 \mathrm{Am}^{2} / \mathrm{kg}$ & Yes $(\sim \mathrm{h})$ & [349] \\
\hline $\begin{array}{c}\text { Nanoporous } \\
\mathrm{Co}_{0.5} \mathrm{Ni}_{0.5} \mathrm{Fe}_{2} \mathrm{O}_{4} \text { and } \\
\mathrm{CoFe}_{2} \mathrm{O}_{4} \text { films }\end{array}$ & $\begin{array}{l}\text { LiTFSI in } \\
\text { EMIM-TFSI }\end{array}$ & M & $\Delta \mathrm{M} \approx 2.5-5 \%$ & Yes $(\sim \min )$ & {$[350]$} \\
\hline
\end{tabular}




\begin{tabular}{|c|c|c|c|c|c|}
\hline $\begin{array}{l}\text { Nanoporous } \alpha- \\
\mathrm{LiFe}_{5} \mathrm{O}_{8} \text { films }\end{array}$ & $\begin{array}{l}\text { LiTFSI in } \\
\text { EMIM-TFSI }\end{array}$ & M & $\Delta \mathrm{M} \approx 4 \%$ & Yes $(\sim \mathrm{h})$ & [351] \\
\hline $\begin{array}{l}\text { Nanoscale } \alpha-\mathrm{Fe}_{2} \mathrm{O}_{3}- \\
\text { based film }\end{array}$ & $\mathrm{LiPF}_{6}$ in $\mathrm{PC}$ & $\begin{array}{l}\mathrm{M}_{\mathrm{S}} \\
\mathrm{H}_{\mathrm{C}}\end{array}$ & $\begin{array}{c}\Delta \mathrm{M}_{\mathrm{s}} \approx 157.14 \\
\mathrm{emu} / \mathrm{g} \\
\Delta \mathrm{H}_{\mathrm{C}} \approx 134.4 \mathrm{Oe}\end{array}$ & Yes & {$[330]$} \\
\hline $\mathrm{Co}_{3} \mathrm{O}_{4}$ film & $\mathrm{PC}$ & $\begin{array}{l}\text { Magnetic } \\
\text { transition }\end{array}$ & FM-PM transition & Yes $(1 \mathrm{~h})$ & [311] \\
\hline $\begin{array}{l}\mathrm{Co} / \mathrm{GdO}_{\mathrm{x}} \text { ultra-thin } \\
\text { films }\end{array}$ & $\mathrm{GdO}_{\mathrm{x}}$ & $\mathrm{H}_{\mathrm{C}}$ & & Yes $(10 \mathrm{~s})$ & [314] \\
\hline $\mathrm{AlO}_{\mathrm{x}} / \mathrm{GdO}_{\mathrm{x}} / \mathrm{Co}$ films & $\mathrm{GdO}_{\mathrm{x}}$ & M & $\begin{array}{c}-80 \% \text { (interface) } \\
-38 \% \text { (bulk) }\end{array}$ & $\begin{array}{l}\text { Partially } \\
\text { (40 min) }\end{array}$ & [315] \\
\hline $\begin{array}{l}\text { Nanoporous } \mathrm{Fe}-\mathrm{Cu} \\
\text { films }\end{array}$ & $\mathrm{PC}$ & $\begin{array}{l}\mathrm{M}_{\mathrm{S}} \\
\mathrm{H}_{\mathrm{C}}\end{array}$ & $\begin{array}{c}\Delta \mathrm{M}_{\mathrm{S}}=20 \% \\
\Delta \mathrm{H}_{\mathrm{C}}=100 \%\end{array}$ & $\begin{array}{l}\text { Partially } \\
\text { (40 min) }\end{array}$ & {$[352]$} \\
\hline $\mathrm{CoN}$ & $\mathrm{PC}$ & $\begin{array}{l}\text { Magnetic } \\
\text { transition }\end{array}$ & FM-PM transition & Yes $(\sim \min )$ & [319] \\
\hline $\mathrm{SmCo}_{5} / \mathrm{Sm}_{2} \mathrm{Co}_{17}$ & Aqueous & $\mathrm{H}_{\mathrm{C}}$ & $\Delta \mathrm{H}_{\mathrm{C}}>10^{4} \mathrm{Oe}$ & & $\begin{array}{l}{[339]} \\
{[340]}\end{array}$ \\
\hline
\end{tabular}

\section{Magnetoelectric devices and systems}

\subsection{Mechanical Antennas}

\subsubsection{VLF Mechanical Antennas}

VLF antennas have unique advantages in underwater and underground communications due to the large electromagnetic wavelength and good penetration distance in dense media. Traditional VLF antennas, however, suffer from relatively large size and power consumption. Recently, mechanical resonators based on ME heterostructures have been utilized for compact and powerefficient antennas. In these devices, the external applied voltage across the electrostrictive/piezoelectric material induces a deformation in this layer and transfers the strain into the magnetostrictive material through ME coupling. The energy minimization taking place in the magnetic material layer drives the magnetization in a periodic manner and generates a nearfield electromagnetic signal for communication along with the piezoelectric phase itself.

In [26], a VLF communication system has been demonstrated based on a pair of ME heterostructures excited at their electromagnetic resonance (EMR) frequency. The structure and the image of devices are shown in Fig. 8(a) and (b), respectively. 3 layers of highlymagnetostrictive Metglas laminates were bonded to a piece of PZT (piezoelectric material) fibers 
with interdigital electrodes (IDE) on it. The bonding and compacting process was conducted in a vacuum bag, and the volume of a single device was $0.33 \mathrm{~cm}^{3}$. The ME transmitter and receiver were tuned by an external DC bias magnetic field of $750 \mu \mathrm{T}$ to resonate at the same frequencies of $23.95 \mathrm{kHz}$. As shown in Fig. 8(c), the ME receiver was able to deliver an output voltage of $215 \mu \mathrm{V}$ with an AC magnetic field of $5.62 \mathrm{nT}$. After measuring the magnetic noise floor at the receiver output by a spectrum analyzer, a $92.3 \mathrm{~dB}$ signal-to-noise ratio was obtained; hence the receiver was calculated to have a magnetic signal minimum detected field (MDF) of $136 \mathrm{fT}$ at EMR. Fig. 8(d) shows the magnetic signal detection measurement results given by a SR830 lock-in amplifier, and the measured MDF is $180 \mathrm{fT}$ at EMR. The communication test was conducted with a driving voltage of $80 \mathrm{~V}_{\mathrm{rms}}$ at $23.95 \mathrm{KHz}$. As shown in Fig. 8(e), the magnetic field generated by the ME transmitter was predicted by a combination of near-field electric dipole and magnetic dipole model, corresponding to the piezoelectric phase and magnetostrictive phase, respectively. It was shown that with a detectivity of $260 \mathrm{fT} / \mathrm{Hz}^{1 / 2}$, a communication distance of $120 \mathrm{~m}$ was achieved, and it was also predicted that a potential communication distance of $2.5^{\sim 10} \mathrm{~km}$ could be achieved with a 100-elements antenna array, using the current receiver setup. The authors also showed a unique modulation scheme (direct antenna modulation, DAM) enabled by the nonlinear response of the devices. Fig. 8(f) indicates that, with a driven carrier signal at EMR frequency and a magnetic modulation signal at baseband frequency of $100 \mathrm{~Hz}$, the antenna itself generated a frequency mixing tone in the spectrum, and a modulation signal to noise ratio (SNR) of $29 \mathrm{~dB}$ was achieved at $16 \mathrm{~m}$ communication distance.

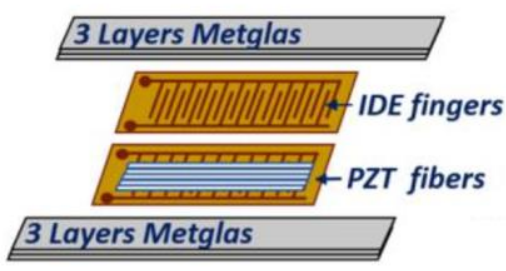

(a)

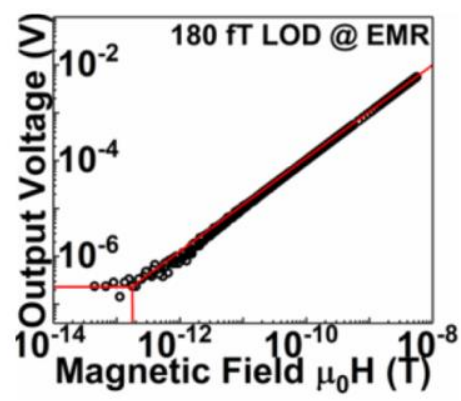

(d)

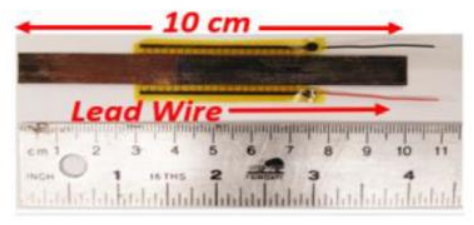

(b)

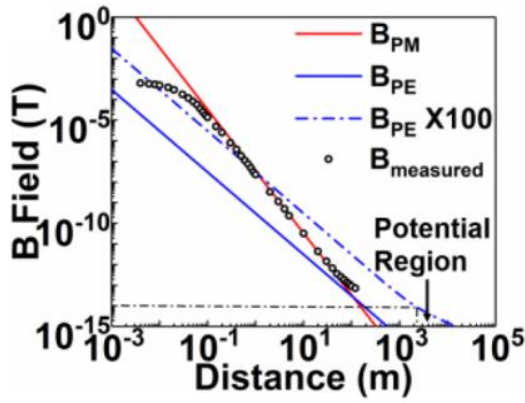

(e)

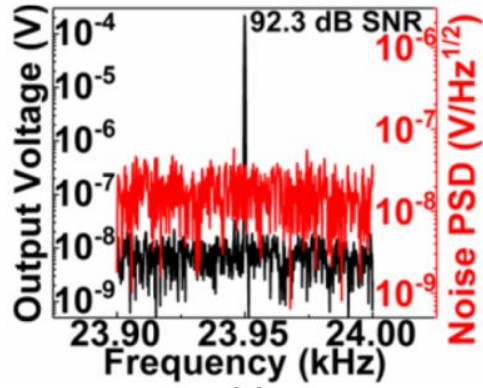

(c)

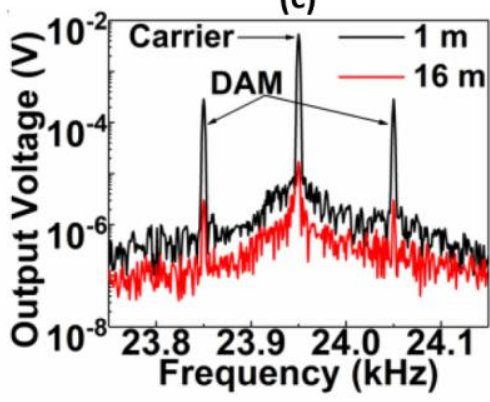

(f)

Fig. 8. (a). Schematic of the ME antenna. (b) Top view of a fabricated ME antenna prototype. (c). Output voltage spectrum of the ME Rx at EMR. (d) MDF measurement of the ME Rx at EMR. (e). Predicted and measured $B$ field distribution along with distance. (f). ME Rx output voltage spectrum shows the received $100 \mathrm{~Hz}$ DAM signal with carrier signal at EMR. Reproduced with permission from C. Dong et al. IEEE Antennas and Wireless Propagation Letters, vol. 19, pp. 398402, 2020 [26]. Copyright [2020], IEEE. 


\subsubsection{MEMS Mechanical Antennas}

Conventional antennas are directly driven by electric current or voltage; hence the antenna size is set to be at least one-tenth of the free space electromagnetic (EM) wavelength to maintain acceptable effective aperture and radiation efficiency for practical applications [353, 354]. Thus, miniaturization is a key challenge for conventional antennas at VHF and UHF. Aside from the conventional methods like shape and geometry optimization, high-contrast material loading, lumped components, metamaterial antennas, and so on, researchers have sought a new approach for antenna miniaturization, and the ME antenna based on mechanical vibration presents a potential solution due to the acoustic wavelength being shorter by five orders compared to EM waves at the same frequency [27]. A novel strain-intermediated ME antenna was theoretically reported by Yao et al. [355] and Domann et al. [356]. In these studies, a more efficient antenna radiation than conventional electric antennas at the first harmonic mode with a comparable antenna size was theoretically proven. By combining with microelectromechanical system (MEMS) technology, the first practical integrated ME antenna was designed and fabricated by Nan et al. in 2017 [25]. Based on the suspended MEMS resonator, the ME antenna features smaller dimensions by two orders than conventional antennas without performance degradation [25].

In [25], based on the vibration mode, the ME antennas can be categorized as a nano-plate resonator (NPR, with a laterally-vibrating mode) or a thin film bulk acoustic resonator (FBAR, with a vertically-vibrating mode) as shown in Fig. 9(a) and (g). For both vibration modes, mechanical strain intermediates between the ME composites, and magnetization rotation is the main radiation source. The radiation cycle is based on converse $\mathrm{ME}$ coupling, in which, the magnetization oscillation in the ferromagnetic layer is excited by the induced mechanical strain from the piezoelectric layer, resulting in EM wave radiation. In the receiving cycle, based on direct coupling, the magnetic field of the EM wave interacts with the ferromagnetic layer, and the strain generated by magnetostriction is transferred to the piezoelectric layer, leading to an ac output voltage. The resonance frequencies are set by the width of the ME heterostructure in NPR mode

and its thickness in the FBAR mode, according to $f_{0, N P R}=\frac{1}{2 W} \sqrt{\frac{E_{e q}}{\rho_{e q}}}$ and $f_{0, F B A R}=\frac{1}{T} \sqrt{\frac{E_{e q}}{\rho_{e q}}}$, respectively, where $W$ is the width of NPR resonator, $T$ is the thickness of FBAR resonator, $E_{e q}$ is the equivalent Young' modulus and $\rho_{e q}$ is the equivalent density of the ME composite, respectively. For the NPR antenna, an RF coil was used to drive the antenna by generating an RF magnetic field at the resonating frequency of the NPR resonator as shown in Fig. 9(b). The induced output voltage is detected by a lock-in amplifier. The input admittance was measured and fitted into the modified Butterwoth-Van-Dyke model. Hence, the quality (Q)-factor of 930 and the electromechanical coupling coefficient of 1.35\% are achieved as shown in Fig. 9(c). In Fig. 9(d), a peak induced voltage of $180 \mu \mathrm{V}$ is observed at resonance, from which a strong ME coupling with a coefficient of $6 \mathrm{kV} / \mathrm{cm} \cdot \mathrm{Oe}$ is calculated. For the FBAR antenna, a phase network analyzer (PNA) was used to measure the two-port S-parameters. A clear resonance peak was detected at $2.53 \mathrm{GHz}$ with a peak return loss $\left(\mathrm{S}_{22}\right)$ of $10.3 \mathrm{~dB}$ as shown in Fig. 9(i). (The inset indicates the thickness resonance mode via a simulation of the displacement profile.) The transmission and 
reception behaviors are represented by the transmission coefficients $\left(S_{12}\right.$ and $\left.S_{21}\right)$ in Fig. 9(j). The maximum antenna gain of $-18 \mathrm{dBi}$ is calculated by the gain-transfer method. Control devices using nonmagnetic materials in lieu of the ferromagnetic layer were also tested to confirm that the induced output voltage and radiation signal are based on ME coupling for both NPR and FBAR antennas, as shown in Fig. 9(f) and (I). The measurement results show that the nonmagnetic resonators have weak coupling and radiation that is two orders smaller in magnitude than the magnetic resonators.

To further improve the radiation performance of FBAR antennas, solidly mounted resonator (SMR) antennas were introduced by Liang et al. in 2020 [357]. The SMR antenna is another FBAR antenna designed by fabricating the FBAR resonator on the top of a Bragg acoustic reflector. The Bragg reflector consists of several periods of low-acoustic-impedance and high-acousticimpedance thin film layers, leading to a high acoustic reflection coefficient. Hence, most of the mechanical vibration energy is reflected back into the resonator instead of being dissipated into the substrate, which translates to stronger ME coupling, higher radiation efficiency and higher antenna gain. A 10-dB gain enhancement was observed from the SMR antenna compared to the suspended FBAR antenna. Additionally, another benefit of SMR structure is the robust device structure. The whole SMR is fixed to the substrate through the Bragg reflector instead of relying on narrow anchors as in the suspended FBAR. Moreover, the inherent thin film stress is also released to the substrate for a flat thin film resonator, leading to more orderly magnetic domains, which also improves the radiation efficiency.

With the antenna volume being smaller by orders of magnitude, ME antennas can be utilized in various applications. For example, a NanoNeuroRFID was designed by Zaeimbashi et al. in 2019 [358], with an operating frequency of $27.55 \mathrm{MHz}$ and a $140 \mathrm{~dB}$ lower path loss than conventional antennas with comparable size. By utilizing both the NPR and FBAR modes, one single ME antenna device can be used for energy harvesting and magnetic field sensing simultaneously [359]. The performance of this ME antenna is 1 to 2 orders of magnitude better than reported micro-coils and shows a low MDF of 470 pT. Moreover, ME antennas are good solutions for wireless implantable medical devices (IMDs). In [360], an ME antenna for the medical implantable communication system (MICS) frequency band was reported with a 1000 times smaller volume compared to state-of-the-art antennas. The small antenna volume benefits to the miniaturization of the whole device. Compared with conventional antennas, the resonance frequency of which is affected by the permittivity of the environment, the resonance frequency of ME antenna remains stable within different human tissues due to its acoustic resonance. 


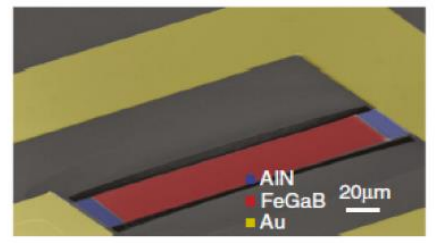

(a)

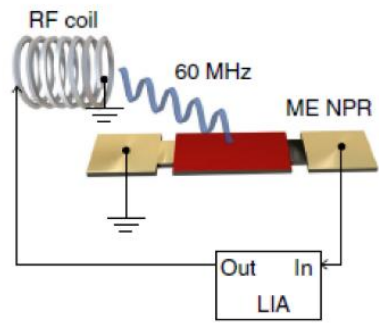

(b)

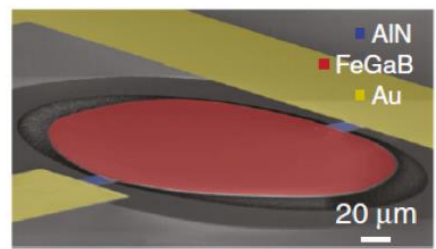

(g)

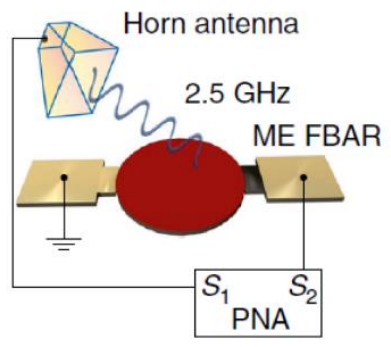

(h)

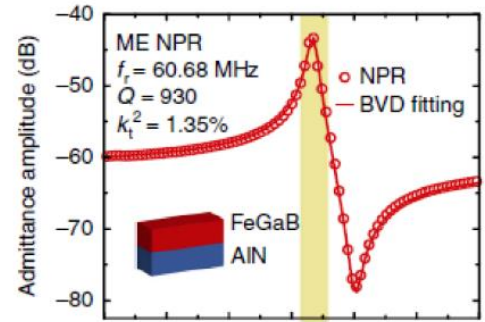

(c)

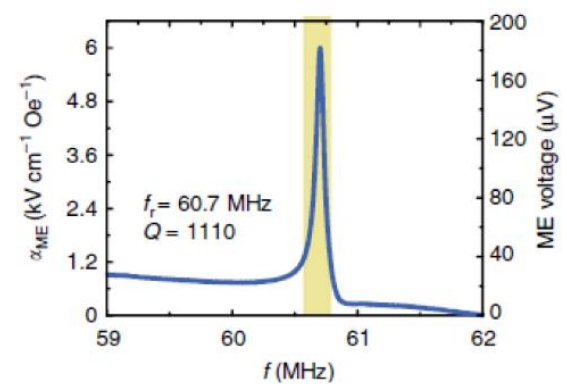

(d)

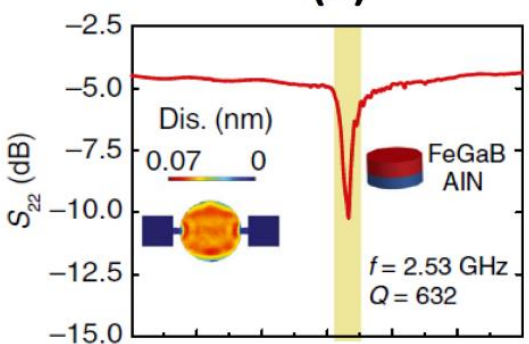

(i)

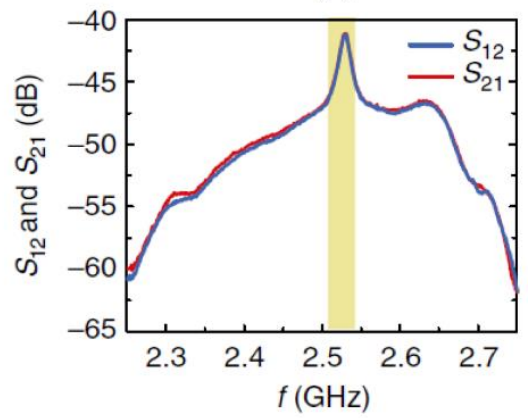

(j)

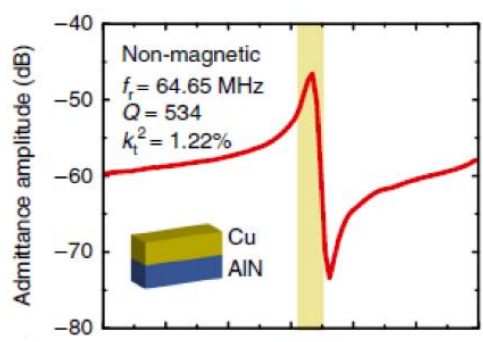

(e)

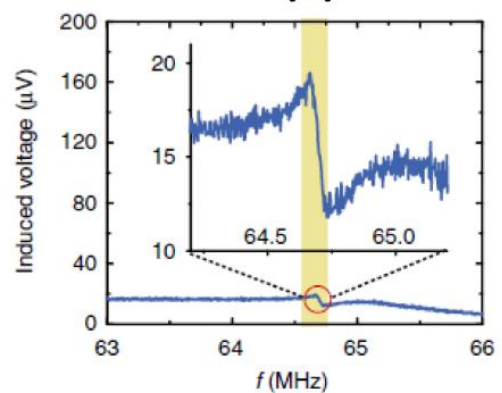

(f)

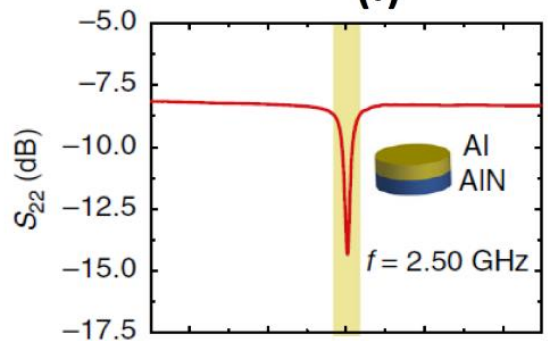

(k)

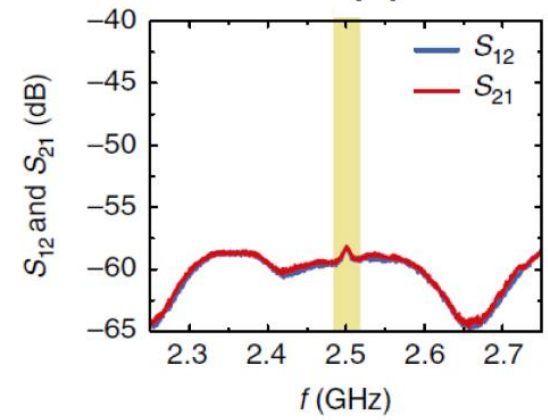

(I)

Fig. 9. NPR antenna: (a) The SEM photo. (b) The measurement setup (c) The amplitude of input admittance and (d) the induced output voltage and calculated ME coefficient at resonance. (e) The amplitude of input impedance and ( $f$ ) the induced output voltage of NPR control device. FBAR antenna: (g) The SEM photo. (h) The measurement setup. (i) The return loss and ( $\mathrm{j}$ ) the radiating signal $\left(S_{12}\right)$ and receiving signal $\left(S_{21}\right)$ at resonance. $(k)$ The return loss, $(\mathrm{I})$ radiating and receiving signal of FBAR control device. Replotted with permission from T. Nan et al. Nature Communications, vol. 8, p. 296, 2017 [25]. Copyright [2017], Nature Research.

\subsection{Magnetic Sensors}




\subsubsection{LF ME Sensors}

Magnetic field sensors have played a significant role in assisting mankind activities including navigation, current sensing, magnetic anomaly detection, mine localization, and biomagnetic measurement, etc. [361]. Different types of magnetometers have been developed during the past century, ranging from the simplest search coil to fluxgate, Hall-effect, magnetoresistance, giant magnetoimpedance, optical pumps, and the most sensitive superconducting quantum interference devices (SQUIDs), among others. Since the invention of multi-phase ME composites, which incorporate both piezoelectric and magnetostrictive components, strong ME coupling has been realized through strain mediation between the two phases [16]. Two-phase ME composites can yield giant ME coupling above room temperature, which enabled technological development for next-generation magnetic sensor applications. Over the last two decades, much effort has been focused on developing various type of ME composite-based magnetic sensors, as they are considered to be promising alternatives for conventional magnetic sensors such as Hall sensors, giant magnetoresistive devices, SQUIDs, etc. [154]. Owing to their simple structure, low power consumption, and room temperature operation, ME sensors have the potential to perform biomagnetic measurements in the field range of 1 to 100 pT at low frequencies. The practical usefulness of ME sensors is determined not only by the output signal in response to an incident magnetic field, but also by the detectivity, together with other important design considerations such as frequency response, dynamic range, linearity, power consumption and spatial resolution.

The mechanism of operation in bulk ME sensors can be categorized into two groups: passive and active detection. Passive detection is based on the direct ME coupling effect, where an ME voltage output is directly generated by the piezoelectric layers due to the strain deformation induced from AC or DC magnetic fields [362]. For practical applications in detecting DC or quasistatic magnetic fields, a charge amplifier is required in this scheme to enhance the SNR and confine the bandwidth between 0.1 to $20 \mathrm{~Hz}$, as the flicker noise in the low frequency range is extremely high. Since 2008, Wang et al. [34] have developed multi-push-pull mode ME laminates consisting of a longitudinally poled piezoelectric PMN-PT single crystal and two symmetric longitudinally magnetized magnetostrictive Metglas layers, as shown in Fig. 10. The symmetric nature allows for optimized elastic coupling between consecutive layers. As a result, a large ME coefficient of $52 \mathrm{~V} / \mathrm{cm} / \mathrm{Oe}$ at $1 \mathrm{kHz}$ (off-resonance) was obtained, as shown in Fig. 11(a). Combined with a low-noise charge amplifier, an extremely low detectivity of $5.1 \mathrm{pT} / \mathrm{Hz}^{1 / 2}$ was achieved at $1 \mathrm{~Hz}$ [132], as shown in Fig. 11(b). However, as the ME coefficient is directly proportional to the piezomagnetic coefficient, a DC bias magnetic field is required to optimize the sensor response, which introduces extra magnetic noise and increases the risk of interference between sensor arrays. Another drawback of the passive detection scheme is the susceptibility to disturbance from vibration sources, as the piezoelectric layer is sensitive to any induced deformations. 


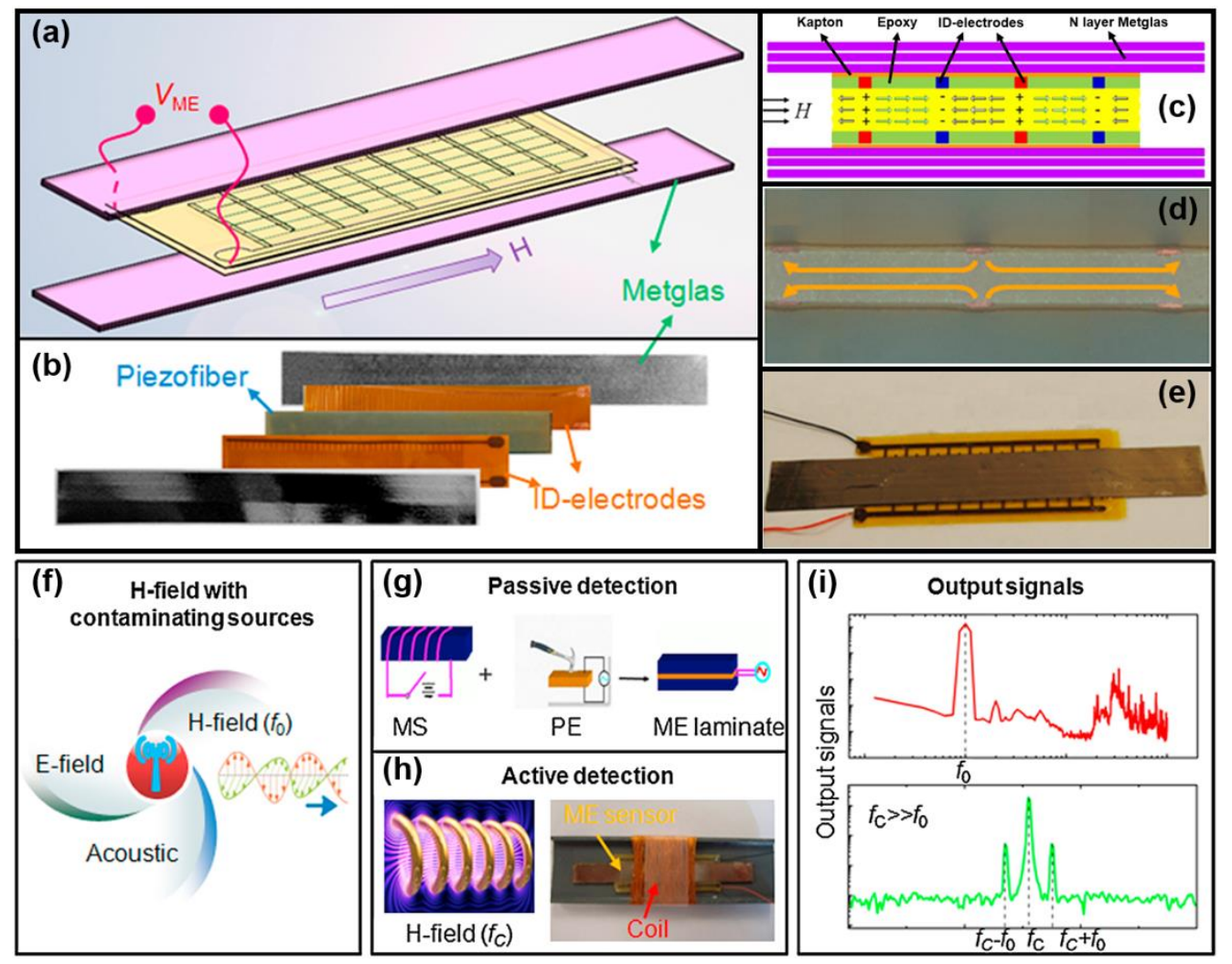

Fig. 10. (a) Schematic diagram of a multi-push-pull configuration ME composite. (b) Exploded view photo of constituent components. (c) Cross-sectional view of the schematic of a multi-pushpull ME composite. (d) Optical micrograph of a longitudinally poled push-pull element in the core composite. (e) Photograph of a multi-push-pull mode ME composite. (f) Fluctuations in low frequency noise and electromagnetic interference make decreasing the noise floor challenging. (g) Working principle of ME sensors in passive detection mode, illustrating that the acoustic and electromagnetic interference induced external noise is inevitable. ( $h$ ) Working principle and prototype ME sensor in active detection mode, in which a modulation field with carrier frequency is applied to the sensor. (i) Output signals from passive and active sensors. Reprinted with permission from Y. Wang et al. Materials Today, 17 (6), 269-275, 2014 [34]. Copyright [2014], Elsevier.

Since the noise level in the low frequency range is relatively high and ME sensors are sensitive to external vibrations, an active detection method based on the magnetic frequency conversion (MFC) has been introduced by Gillette et al. [363]. The principle of the MFC technique lies in the nonlinearity of the magnetostrictive layers, which have a quadratic response to AC magnetic field of small amplitude [364]. Given an applied alternating pumping magnetic field at the mechanical resonance (carrier frequency), a product term will be generated by the modulation of the pumping field and the signal field, which creates a frequency conversion effect [365]. By shifting the low frequency magnetic signals to the sideband of the carrier frequency, this active detection scheme offers the possibility to reject low-frequency environmental noise and can achieve resonance-enhanced sensitivity. Using the same multi-push-pull configuration and the Metglas/PMN-PT based ME sensor, Liu et al. have demonstrated extremely low MDF of 200 pT, 
150 pT, and 20 pT at $0.01 \mathrm{~Hz}, 0.1 \mathrm{~Hz}$ and $1 \mathrm{~Hz}$, respectively [366], as shown in Fig. 11(c) and (d). Although the active detection scheme does not need a bias magnetic field and can effectively circumvent the flicker noise, an extra excitation coil and AC driving current is required, which increases the power consumption and additional noise from the modulation field.

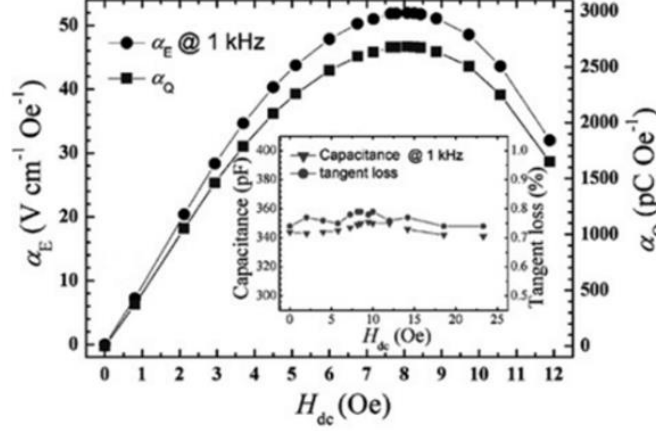

(a)

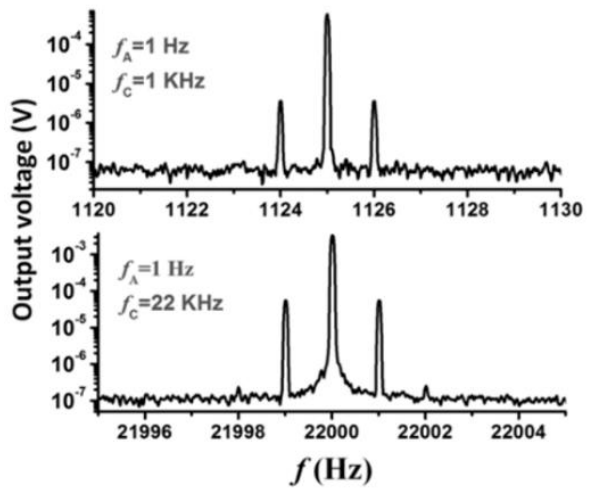

(c)

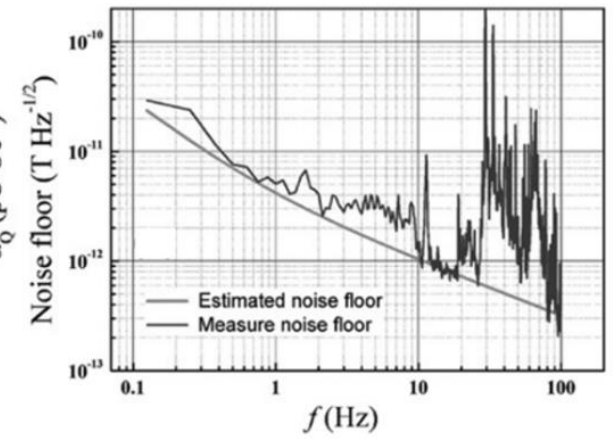

(b)

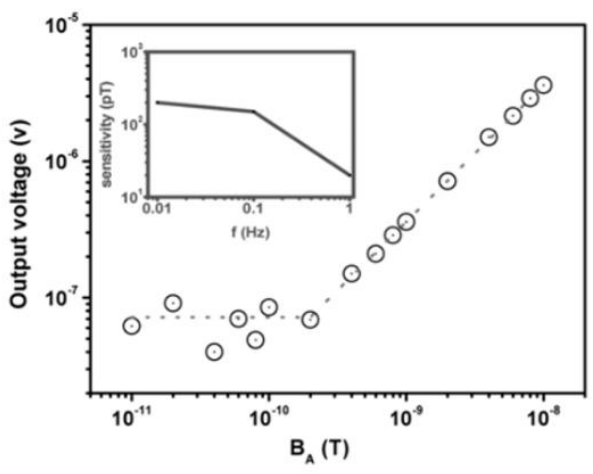

(d)

Fig. 11. (a) The ME voltage coefficient and ME charge coefficient of the passive ME sensor. The inset shows the capacitance and dielectric loss as a function of DC magnetic field. (b) Measured and estimated detectivity of the passive ME sensor unit. (c) Test results of the active ME sensor driven by carrier fields of $1 \mathrm{kHz}$ and $22 \mathrm{kHz}$ to detect incident magnetic fields at $1 \mathrm{~Hz}$. (d) MDF of the active mode ME sensor at $0.01 \mathrm{~Hz}$. The inset gives the MDF at other frequencies. (Note that "sensitivity" has been redefined as "MDF" here.) Reprinted with permission from Y. Wang et al. Advanced Materials, 23(35), 4111-4114, 2011 [132]. Copyright [2011], Wiley-VCH. Reprinted with permission from Y. Liu et al. Applied Physics Letters, 103(21), 212902, 2013 [366]. Copyright [2013], AIP Publishing.

In principle, any ME device exhibiting an ME effect is capable of being directly operated as a magnetic field sensor [15]. This direct ME effect is significantly enhanced in ME composites [34, $143,154,367$ ] of distinct piezoelectric and magnetostrictive materials compared to single phase multiferroics, which inherently present a trade-off between magnetic and dielectric polarizability [148]. Ideally, such an ME composite magnetic field sensor is capable of directly transferring a magnetic field signal into a proportional voltage signal at the electrodes of the piezoelectric material phase, the effect of which is related to the ME coefficient $\alpha_{\text {Direct. }}$ Other possible ME magnetic field detection schemes are based on surface acoustic wave (SAW) devices, which make use of the velocity dependence of those waves on the magnetic media they propagate. 
Overall, the ME coefficient of two-phase composite materials as a figure of merit can be described by a product rule [368] $\alpha_{\text {Direct }}=\frac{\partial P}{\partial H}=\frac{\partial P}{\partial \sigma} \times \frac{\partial \sigma}{\partial \lambda} \times \frac{\partial \lambda}{\partial H}$, where the change of magnetostriction $\lambda$ with alteration of the magnetic field $H$ equals to the magnetostrictive susceptibility $\partial \lambda / \partial H$. Together with the transduction of magnetostrictive strain to mechanical stress $\partial \sigma / \partial \lambda$ in the piezoelectric component and the change of polarization with mechanical stress $\partial P / \partial \sigma$, a response of the magnetostrictive phase results in a detectable change of polarization with field $\partial P / \partial H$ in the piezoelectric phase. By this, the overall performance of $M E$ field sensors depends on the properties and the response of all the individual components in the multi-phase sensor.

In general, in ME sensors, low-frequency magnetic signals might be transferred unsatisfactorily and are subject to piezoelectric leakage, non-ideal mechanical coupling between the material phases and associated voltage drift. Furthermore, $1 / f$-noise strongly elevates the noise floor at frequencies below a few hundred Hertz [369, 370]. Materials with favorable properties for mitigating these limitations, often utilized in ME thin-film sensor structures, include AIN [61, 144] or AIScN [371, 372] for the piezoelectric phase and a soft-magnetic magnetostrictive material for the piezomagnetic phase. Cantilever-based thin film ME sensors generally consist of micromachined Si cantilevers coated with the piezoelectric and magnetostrictive layers [373]. For the piezoelectric material, large ratios of the piezoelectric voltage coefficient together with a high dielectric constant and its loss tangent $\tan \delta$ are of relevance. These properties favor the beforementioned $\mathrm{Al}(\mathrm{Sc}) \mathrm{N}$ piezoelectrics. Alternative designs might use additional piezoelectric layers [374]. In combination with the piezomagnetic phase also a low processing temperature is of importance [61]. For alternative modes of operation or sensor designs, like SAW-based sensors [375], bulk piezoelectric materials like quartz, also in combination with ZnO films [376], are utilized. For the magnetostrictive phase, a high piezomagnetic coefficient at the working point of the sensor is an apparent requirement, favoring soft-magnetic amorphous thin films like FeCoSiB [377], FeGaB [378], FeGaC [379], FeCoC [380] or similar soft-magnetic alloys with high saturation magnetostriction for ME sensor applications. Laminating the materials in thin-film sensors provides an additional option to improve the soft-magnetic characteristics, allowing also for the reduction of eddy currents by laminating with an insulator. The use of magnetostrictive multilayers further enables the design of effective magnetic parameters at will. Magnetostrictive ferromagnetic/antiferromagnetic multilayers, such as FeCoSiB/MnIr [381, 382], allow for self-biased ME sensors, enabling also magnetic vector field sensing capabilities [383]. Moreover, it facilitates the reduction of the effects of magnetic domain activity [384-386], thereby reducing magnetic noise [387] in the piezomagnetic phase.

We first discuss cantilever-based sensor approaches. Commonly, for such sensors the ME coefficient relevant for the application is determined at the working point of the composite, which is mainly given by two factors: first, a static bias field $\left(H_{\text {bias }}\right)$, required to set the maximum piezomagnetic coefficient, i.e., the highest slope of the magnetostrictive response and, second, a mechanical resonance which is matched by the external magnetic field $\left(H_{\text {ext }}\right)$ frequency. Fig. 12(a) shows a schematic direct ME field sensor setup. When applying AC magnetic signals to the 
composite, its response will be modified by mechanical vibration resonances $\left(f_{0}\right)$, which typically lead to a strong ME effect enhancement (Fig. 12(b)) given by the $Q$ factor at the specific mechanical resonance. The interplay of an excited resonance, with respect to the electrodes placed on the piezoelectric material, together with the exhibited $1 / f$ noise (Fig. 12(c)), then determines the resulting enhancement on the sensing performance $[61,388,389]$. Thus, a small-amplitude wideband signal has a sufficiently high SNR in a very narrow frequency range around the mechanical resonance (Fig. 12(d)). In the shown case, the detectivity is enhanced by several orders of magnitude at the mechanical flexural resonance. Below the resonance, noise significantly deteriorates the sensor response. To benefit from this strong SNR enhancement occurring in passive direct ME sensors, several routes for bandwidth enhancement have been developed in recent years with the goal of measuring low frequency magnetic field signals.

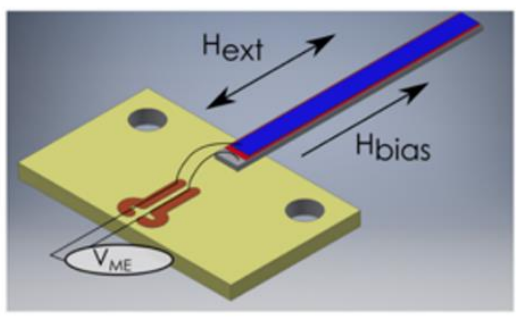

(a)

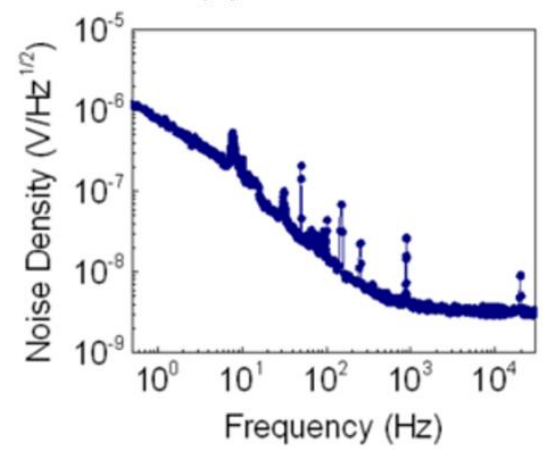

(c)

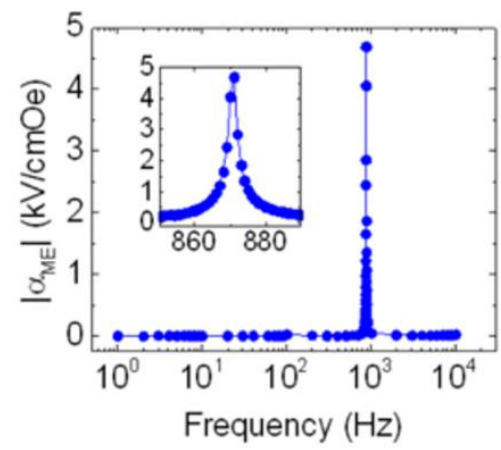

(b)

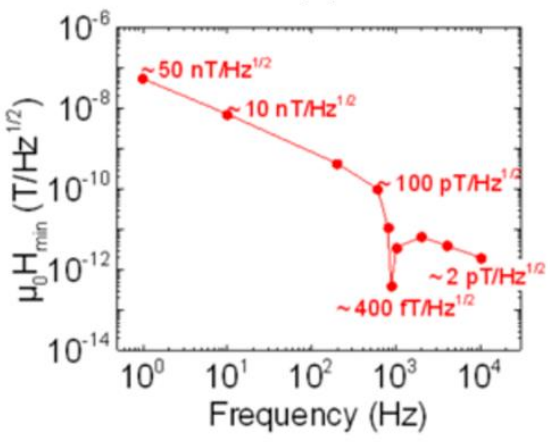

(d)

Fig. 12. (a) Schematic of a cantilevered silicon based thin film ME sensor, the freestanding length is about $20 \mathrm{~mm}$. (b) Magnitude of ME coefficient $\alpha_{\text {ME }}$ of a composite AIN-FeCoSiB-based resonator as a function of magnetic signal frequency. The inset shows the values around the cantilever flexural resonance slightly below $1 \mathrm{kHz}$. (c) Corresponding noise voltage density and (d) resulting detectivity versus signal frequency. Towards DC the noise is increased by about 5 orders of magnitude. The minimum detectivity corresponds to the effect enhancement at the mechanical resonance. Replotted with permission from E. Yarar et al. Applied Physics Letters 109.2: 022901,2016 [61]. Copyright [2016], AlP Publishing.

Magnetic Frequency Conversion (MFC) requires active excitation of the ME composite by means of an external AC magnetic field, commonly termed as a pumping field. The external pumping 
field frequency $\left(f_{\text {pump }}\right)$ is set slightly off-resonance, for the condition $f_{\text {ext }}=f_{0} \pm f_{\text {pump }}$ to be met [390]. This effectively shifts the desired low-frequency signal into the narrowband resonance peak at $f_{0}$, given that the magnetic field frequency is low $\left(f_{\text {ext }}<f_{\text {pump }}\right)$. The ME composite then effectively acts as a frequency mixer and narrowband amplifier. Results obtained by $[369,391]$ indicate a (low-frequency) SNR improvement of about two to three orders of magnitude with respect to the same composite used under passive, i.e., direct effect conditions. Low-noise optimized MFC can reach detectivities of $60 \mathrm{pT} / \mathrm{Hz}^{1 / 2}$ at $10 \mathrm{~Hz}$ [392] for improved sputtered FeCoSiB-based multilayers and about $130 \mathrm{pT} / \mathrm{Hz}^{1 / 2}$ at $1 \mathrm{~Hz}$ [393] using Metglas foils. For this method to apply to wideband signals, the pumping signal is swept in order to meet equation of $f_{\text {ext }}$ for a multitude of frequencies. This in turn can be thought of as a magnetic spectrum analyzer [394]. The main technical challenges and limitations for applications using this approach come from the requirement for a magnet coil in the vicinity of the composite to supply the magnetic pumping signal and the rather high energy demands for driving the coil.

Electrical frequency conversion (EFC) aims to remedy the requirement for driving an external coil by supplying the equivalent of the pumping field using a piezoelectric material, which exerts periodic stress on the magnetostrictive material, thus enabling a similar modulation approach as in the case of MFC $[374,395]$. The absence of an active driving electromagnetic coil makes this method readily integrable into volume devices. However, specific drawbacks are the requirement of large dynamic ranges of the output electronics because the piezoelectrically supplied carrier signal strongly feeds through to the output, contaminating the magnetic signal information. EFC using piezoelectric readout so far reaches detectivities in the low $\mathrm{nT} / \mathrm{Hz}^{1 / 2}$ regime $[374,395]$. Nevertheless, replacing a magnetic field with a piezoelectric stress is not straightforward, because a magnetic field contributes unidirectionally to the magnetic energy landscape. Stress, on the contrary, leads to a uniaxial anisotropy, altering the magnetic energy landscape in a symmetric manner, thus also leading to more complicated magnetic reversal patterns [396, 397].

In principle, all the modulation techniques benefit from high mechanical $\mathrm{Q}$ factors. This in turn inherently limits the sweep speed to, in practice, a few times the sensor bandwidth $B=Q \cdot 1 / f_{0}=$ $Q \cdot t$. For any oscillator, settling time is at least $Q$ oscillation periods of time $t$. Consequently, when $Q$ is very high, $f_{0}$ should also be very high in order to reach a sufficiently high sensing bandwidth $B$. This approach of satisfying the equation of $f_{\text {ext }}$ is only necessary if the frequency of the field signal of interest $f_{\text {ext }}$ is significantly larger than the bandwidth of the employed mechanical resonance mode. Otherwise, the benefit of resonance amplification for one of the formed sidebands is negligible and the excitation at the resonance itself is preferable.

Thus, one alternate approach was developed that is based on the use of piezoelectric excitation combined with an inductive signal pick-up (Fig. 13). This scheme is especially effective using high frequency resonances as the induced voltage in the coil $V_{\text {coil }}$ scales with the rate of change of the magnetic flux $V_{\text {coil }} \propto \mathrm{d} \phi / \mathrm{d} t$. Consequently, the induced voltage is proportional to the piezoelectric pump frequency $f_{\text {pump, }}$ which then leads to very high magnetic field sensitivities. For such a measuring scheme, the mechanical resonance conditions are of importance. Mechanical resonance frequencies (i.e., flexural, torsional) typically scale inversely with resonator dimensions, making smaller dimensions beneficial for achieving high frequency resonances. On 
the other hand, magnetostrictive soft magnetic films typically show a distorted magnetic anisotropy landscape towards their film edges [385, 398]. Thus, extended films facilitate the control of magnetic anisotropy. The use of bulk modes such as thickness or longitudinal oscillations is another alternative to achieve high mechanical frequencies while maintaining larger resonator geometries [399].

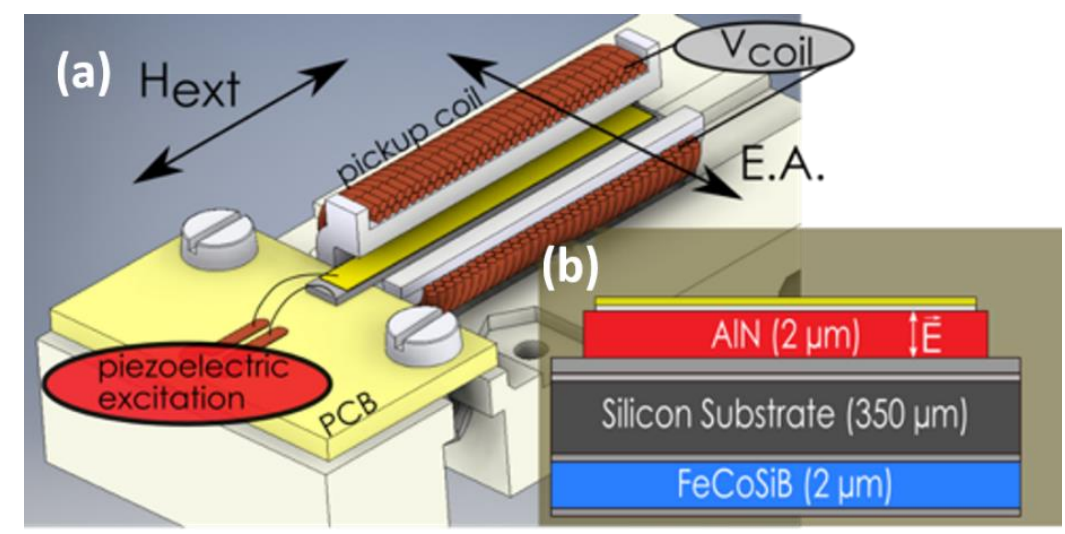

Fig. 13. Setup using the converse ME effect with thin film-based composites. (a) Resonator employing the converse ME effect using thin films. An excitation voltage is applied to the piezoelectric material, exciting a mechanical resonance, the induced pickup coil voltage at the excitation frequency is acquired through the pickup coil. The external magnetic field $\left(\mathrm{H}_{\text {ext }}\right)$ is applied perpendicular to the magnetic easy axis (E.A.). This pickup coil voltage is a function of $\mathrm{H}_{\text {ext. }}$ (b) Cross-section of the ME composite.

Fig. 14(a) shows example data from such a device. The induced coil voltage $\left(V_{\text {coil }}\right)$ amplitude is monitored while piezoelectrically exciting a high frequency mechanical resonance at $514.8 \mathrm{kHz}$ with respect to a quasi-static external magnetic field $H_{\text {ext. }}$. At external field magnitudes above $100 \mu \mathrm{T}$ the voltage drops rapidly as the amorphous thin film FeCoSiB approaches magnetic saturation. Depending on the magnetic history, points of maximum $V_{\text {coil }}$ are located near a field magnitude of 30 and $50 \mu \mathrm{T}$. For sensing purposes, i.e., best detectivity, the situation at zero bias field, coming from any saturation direction is favorable, because a linear slope of up to $40 \mathrm{kV} / \mathrm{T}$ is present even using low excitation voltage of $80 \mathrm{mV}$ (Fig. 14(b)). Note that at higher bias fields this slope is reduced, by which the sensing performance is slightly degraded. Furthermore, if such ME resonators are strongly driven, mechanical nonlinearities may occur, which also degrade the sensing performance or make control difficult [400]. 


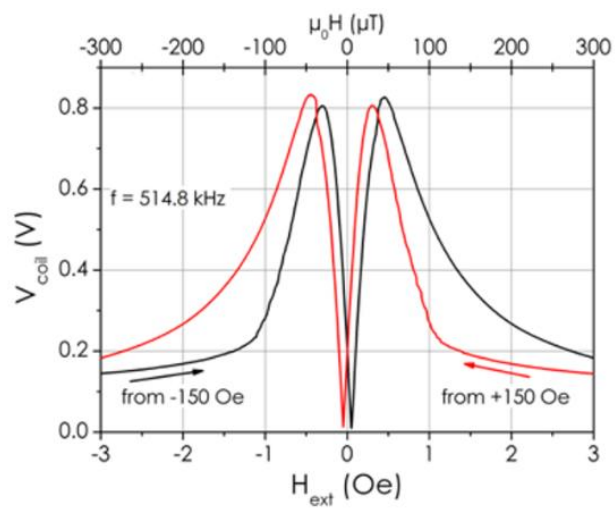

(a)

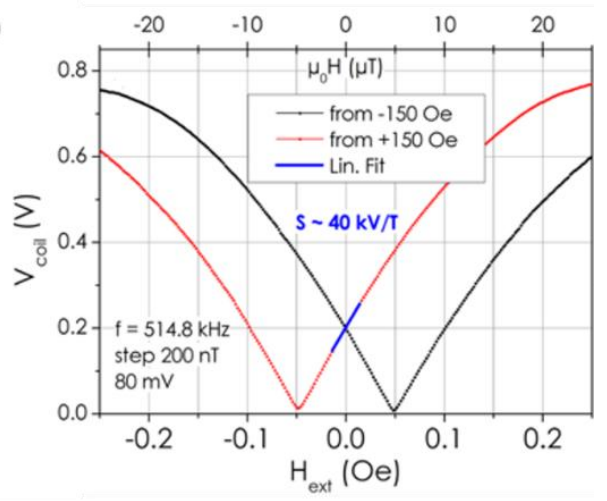

(b)

Fig. 14. (a) The induced coil voltage $\left(V_{\text {coil }}\right)$ at the excitation frequency of $514.8 \mathrm{kHz}$ with respect to an external magnetic field. The two loop branches show data for ascending and descending magnetic fields starting from magnetic saturation. At very high external fields the induced voltage reaches minimum. At an intermediate external field magnitude, depending on the chosen working point, a maximum of $V_{\text {coil }}$ is reached, indicating strong emittance of the resonator. (b) Around zero external bias fields, the sensitivity, i.e., the slope of the curve reaches a maximum in magnitude. This is the optimum working point for use as a converse ME sensor. Reprinted with permission from P. Hayes et al. Scientific reports 9.1, 1-10, 2019 [401]. Copyright [2019], Nature Publishing Group.

This scheme provides detectivities away from the carrier frequency of down to about $50 \mathrm{pT} / \mathrm{Hz}^{1 / 2}$, increasing to about $120 \mathrm{pT} / \mathrm{Hz}^{1 / 2}$ at $2 \mathrm{~Hz}$ (Fig. 15(a)). The sensitivity within the bandwidth $B$ of the resonator remains practically constant. A noise increase close to the mechanical resonance leads to degraded detectivities at low frequencies of $f_{\text {ext }}$. Ultimately, the DC response at varying external fields, which is given by the amplitude of $f_{\text {pump }}$ itself, is shown in Fig. 15(b). From the base noise with constant amplitude and the respective step height, a detectivity of about $210 \mathrm{pT} / \mathrm{Hz}^{1 / 2}$ for DC fields is obtained [401].

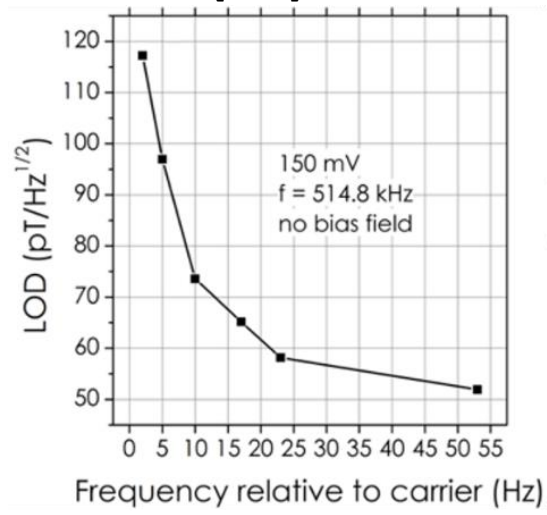

(a)

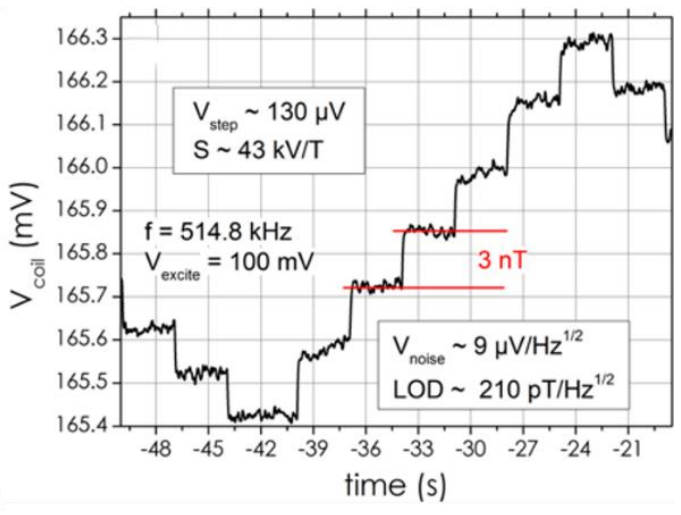

(b)

Fig. 15. (a) Detectivity with respect to the carrier frequency. The sensitivity stays constant within the shown frequency regime, a strong drop in noise is responsible for the improved detectivity away from the carrier frequency $f_{\text {pump. }}$ (b) Staircase DC measurement using an electrically modulated composite at zero magnetic bias field. The sensor is piezoelectrically excited within a 
high frequency resonance using an inductive pickup. Recording of the coil voltage at the excitation frequency against time reveals a high DC magnetic field detectivity of about 210 $\mathrm{pT} / \mathrm{Hz}^{1 / 2}$. Note: the terminology "LOD" is redefined as "detectivity" here. Reprinted with permission from P. Hayes et al. Scientific reports 9.1, 1-10, 2019 [401]. Copyright [2019], Nature Publishing Group.

An alternative approach are ME sensors based on surface acoustic waves (SAWs). For that concept, metallic interdigital transducers (IDTs) are deposited on a piezoelectric single crystal substrate (e.g., quartz, $\mathrm{LiNbO}_{3}, \mathrm{LiTaO}_{3}$ ) and are used to excite high frequency elastic waves in the $\mathrm{MHz}$ to low $\mathrm{GHz}$ regime. If these high frequency waves travel through a magnetostrictive material, their phase velocity is directly proportional to the change of the Young's ( $\Delta \mathrm{E}$ effect) or the shear ( $\Delta G$ effect) modulus with magnetic field-induced changes in magnetization. The concept of magnetically changing the phase of an acoustic wave was first experimentally demonstrated by Ganguly et al. [402] using Rayleigh wave-based devices. However, the achieved phase changes were comparably low at high magnetic bias fields, thus unsuitable for magnetic field sensing. In recent years, the interest in using SAW devices as magnetic field sensors has increased, with several groups investigating different designs and materials [375, 403-407]. It turned out that the highest magnetic sensitivities can be achieved by employing a guiding layer on substrates which generate shear horizontal waves, thereby exciting so-called Love waves [375, 376, 408, 409]. Love waves are strongly confined to the surface of the guiding layer. Hence, most of the acoustic energy is focused in the magnetostrictive material (cf. Fig. 16(a)). Additionally, due to the horizontal shear displacement of the wave it is mostly sensitive to changes of the shear modulus of the magnetostrictive medium.

Love wave sensors using gold IDTs on a ST-cut Quartz substrate, with a $4.5 \mu \mathrm{m}$ thick $\mathrm{SiO}_{2}$ guiding layer and a $200 \mathrm{~nm}$ thick magnetostrictive phase of composition ( $\left.\mathrm{Fe}_{90} \mathrm{CO}_{10}\right)_{78} \mathrm{Si}_{12} \mathrm{~B}_{10}$ can exhibit phase sensitivities as high as $2000^{\circ} / \mathrm{mT}$ (Fig. 16(b)) and detectivities as low as $70 \mathrm{pT} / \mathrm{Hz}^{1 / 2}$ at $10 \mathrm{~Hz}$ and $25 \mathrm{pT} / \mathrm{Hz}^{1 / 2}$ at $100 \mathrm{~Hz}$ (Fig. 16(c)) [410]. Since SAW magnetic field sensors operating in a delay line configuration are not dependent on any resonance effects, their measurement bandwidth is solely limited by the propagation time of the acoustic wave and the passband width of the device. Depending on the design, i.e., delay line length and wavelength, bandwidths of up to $1 \mathrm{MHz}$ can be achieved making them particularly interesting for artificial current sensing [411]. Higher order Love modes can also be excited, but generally show lower sensitivities than the fundamental mode [412]. However, with a guiding layer to wavelength ratio of about 3/2, three different modes can be excited simultaneously, while providing sufficient sensitivities. This way, multimode operation is enabled. In general, the sensitivity of Love wave magnetic field sensors is proportional to the thickness of the magnetostrictive phase, i.e., the thicker the magnetostrictive layer, the higher the sensitivity. The generated magnetic noise, however, shows the same dependency and therefore in a thickness range of $50 \mathrm{~nm}$ to $250 \mathrm{~nm}$ the resulting detectivity is virtually the same [413]. 


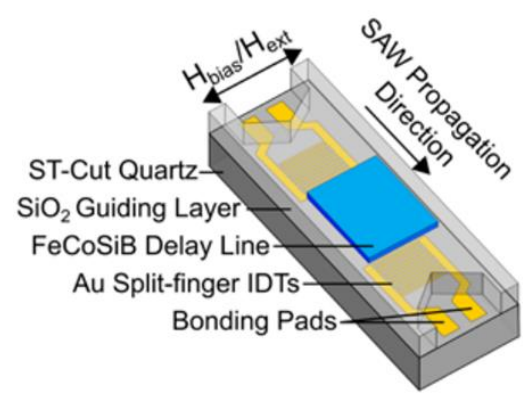

(a)

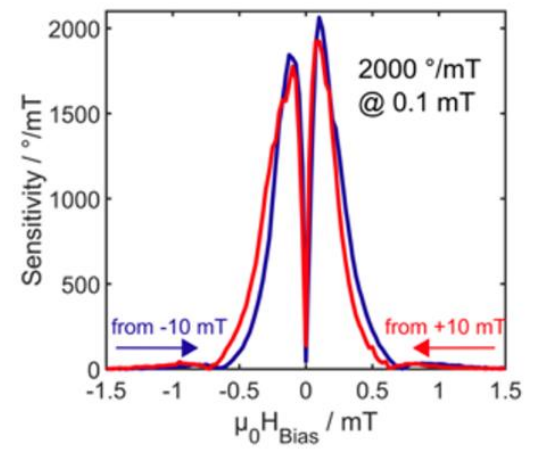

(b)

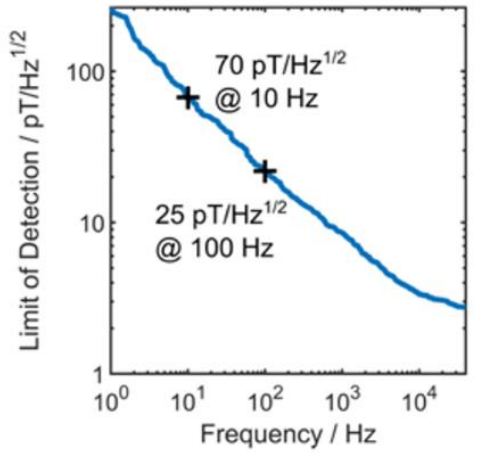

(c)

Fig. 16 (a) Schematic of magnetic SAW delay line sensor. Two pairs of interdigital electrodes are deposited on a piezoelectric substrate which send off and receive a surface acoustic wave at the in- and output, respectively. By use of a guiding layer, the energy of the wave is confined to near

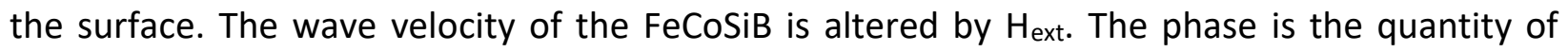
measurement. (b) Magnetic sensitivity with applied magnetic DC bias field. (c) Detectivity over a frequency range of a $40 \mathrm{kHz}$ from the carrier signal $(148 \mathrm{MHz})$. Note: the terminology "Limit of Detection" is redefined as "Detectivity" here. Replotted with permission from V. Schell et al. Applied Physics Letters 116.7, 073503, 2020 [410]. Copyright [2020], AIP Publishing.

The detectivity in the ME sensors discussed so far is limited by different noise sources. A fundamental limitation comes from thermal-mechanical noise of the resonator [390, 414] and thermal-electrical noise of the piezoelectric material, where applicable. Yet, theoretical and experimental analysis shows that magnetic losses or hysteresis effects, mostly as a result of magnetic domain wall processes, are the dominating contributions to sensor noise in $\mathrm{ME}$ structures. A direct implication of complex and hysteretic magnetic domain response on ME sensitivity, thereby directly coupling into ME signal variations, was determined in [385]. For modulated sensor structures, different regimes of magnetic noise with distinct magnetic domain activities could be identified [386]. The connections between sensor sensitivity, magnetic noise, detectivity, and magnetic domain induced losses for a MFC operated sensor are shown in Fig. 17. The individual magnetic domain activities could be directly connected to magnetic loss parameters. Similar relations were found for SAW-based devices, where the main factor limiting the detectivity is the $1 / f$ magnetic flicker phase noise stemming from the acoustically penetrated magnetic FeCoSiB film. This is particularly evident when the SAW sensors are operated in magnetic saturation. In this case, the noise floor is reduced by up to 2 orders of magnitude as compared to operating the sensor at its point of highest sensitivity [415]. 


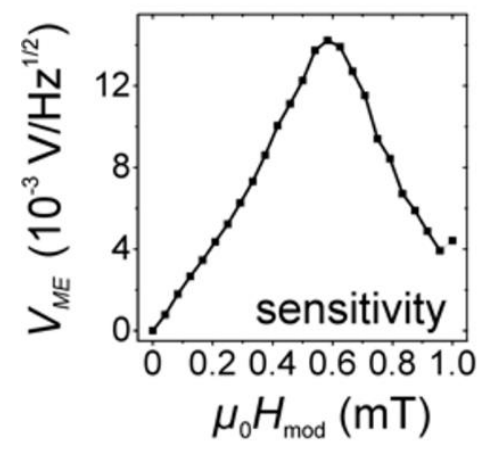

(a)

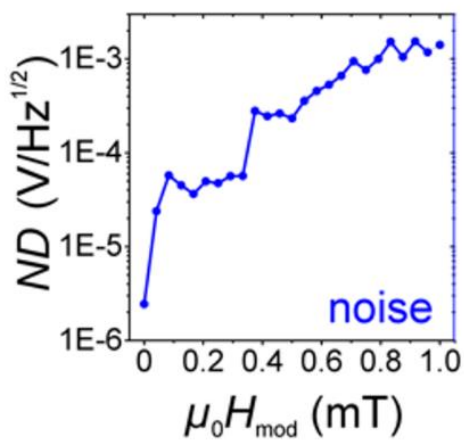

(b)

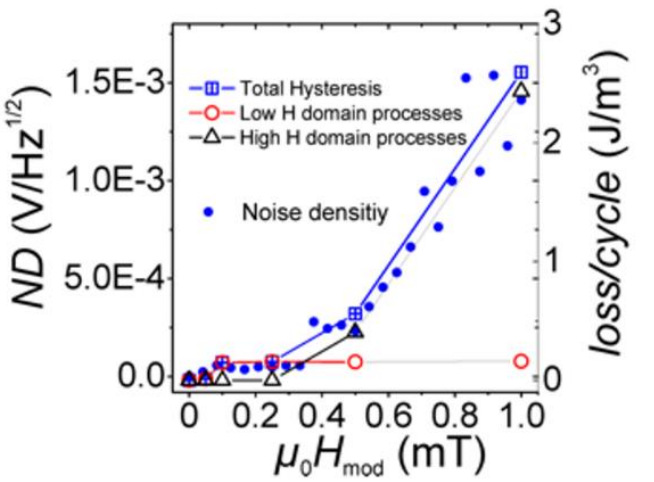

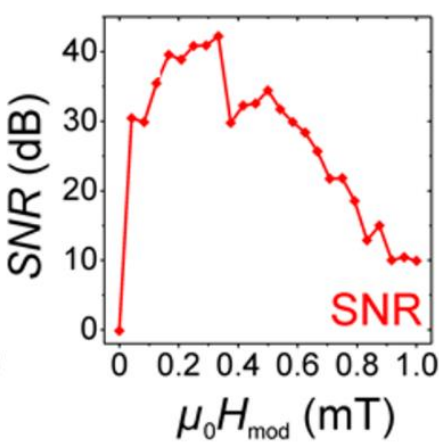

(c)

(d)
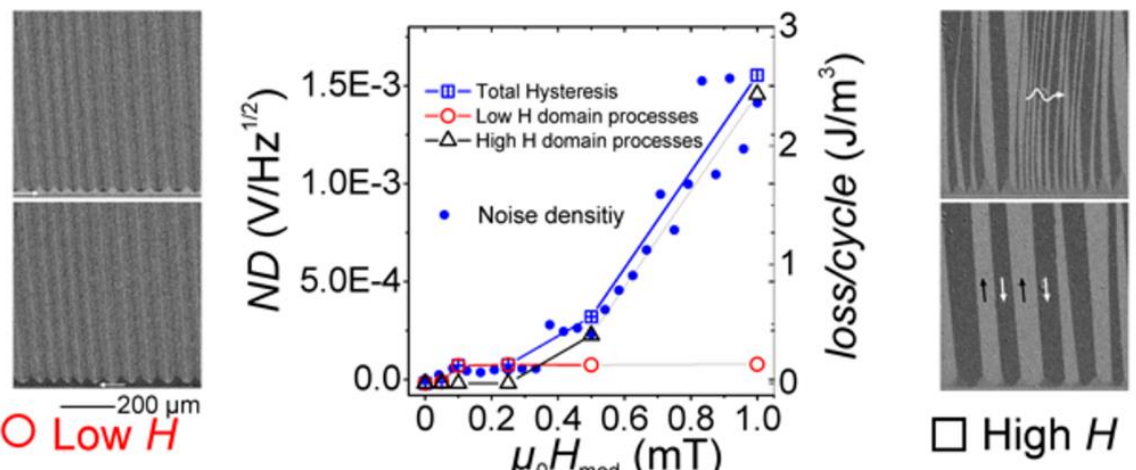

Fig. 17 Variation of MFC sensor properties with modulation field $H_{\text {mod. }}$ (a) ME voltage signal amplitude of an MFC sensor (max. at $\mu_{0} H_{\text {mod }}=0.6 \mathrm{mT}$ ) and (b) corresponding ME voltage noise density (ND) for a measurement signal $\mu_{0} H_{\text {mea }}=10^{-3} \mathrm{mT}$ at a frequency of $f_{\text {mea }}=10 \mathrm{~Hz}$. Magnetic noise density is non-linear increasing with increasing $H_{\text {mod. }}$ (c) Resulting values of SNR with best SNR away from maximum sensitivity. (d) Direct correlation of low and high magnetic field induced magnetic domain activity, magnetic hysteresis losses, and noise density. Reprinted with permission from N. Urs et al. Physical Review Applied 13.2, 024018, 2020 [386]. Copyright [2020], American Physical Society.

As for cantilever-based sensors, the noise and not the sensitivity is the dominating factor for sensor improvement. For cantilever-based sensors operated by MFC, magnetic domain control was already achieved by quasi-elimination of magnetic domain activity and magnetic noise using an antiparallel exchange biasing scheme in a magnetic multilayer piezomagnetic phase [387, 392]. This way, single magnetic domain structures are formed in each layer, and magnetic noise could be (nearly) eliminated.

One of the major challenges for improving the ME characteristics for future devices from the point of view of the magnetic phase lies in the control of magnetic properties. Four major requirements related to the magnetic phase are obvious for obtaining the best ME response for the achievement of low detectivity. First, increasing the saturation magnetostriction constant of the magnetic materials is one basic prerequisite for increased sensor ME signals. Second, the exact alignment of the magnetization in the sensors, most relevant for obtaining high signal amplitude and thus maximum sensitivity, is of high relevance. Even small misalignment in the 
anisotropy axis or local anisotropy dispersion degrades the maximum achievable sensitivity in the sensors. Related to this are inhomogeneous demagnetization field contributions in structured sensors as well as stress relaxation effects at the edges of the magnetostrictive structures. Third and relatedly, the magnitude of effective magnetic anisotropy plays a significant role for obtaining high magnetostrictive susceptibility. All these contributions, especially with the inevitably occurring (local) magnetoelastic anisotropy effects in the magnetic devices, will also determine the magnetic domain structure in the sensors. As all the presented ME sensor schemes rely on modulation and thereby activate magnetic domain wall processes, the overall magnetic domain activity has a major influence on the sensor noise characteristics. Therefore, and fourthly, magnetic domain control with the goal of eliminating magnetic domain wall activity is of major relevance to limit or eliminate magnetic sensor noise. This strategy is at the heart of commercial magnetic film sensors, e.g., for AMR-based magnetic sensor structures [416, 417]. Thus, the focus of further research with the goal of achieving the lowest detectivities, e.g., for biomedical applications, is the reduction of magnetically generated noise. Magnetic anisotropy control together with magnetic domain engineering is a key aspect for sensor improvement. By implication, this requirement will put constraints on the range of compatible levels of magnetostriction and magnetic anisotropy, also in combination with sensor processing conditions. As already demonstrated, magnetic multilayers offer a lever for magnetic domain control. With this, the final goal for all the presented sensor schemes is to reach theoretical thermomagnetic noise limits and thus detectivities in the sub-pT magnetic field regime for the detection of low-frequency magnetic fields.

Overall, the presented sensor structures allow ME composites to be successfully implemented as sensing devices in conjunction with low-cost state-of-the art signal processing schemes. Further improvements in sensor structures, materials, and optimization of magnetics and electronics will enable sensors with significant lower detectivities and higher bandwidths.

\subsubsection{MEMS ME Sensors}

ME nano/micromechanical systems (NEMS/MEMS) resonant magnetometers operating on the $\Delta \mathrm{E}$ effect provide advantages of small scale and low cost. With dimensions of the resonator plate of roughly one or two hundred microns across and hundreds of nanometers thick, and made using microfabrication techniques, hundreds of devices can fit on a single Si substrate chip. The small size enables high spatial resolution, if the sensors are used in an array, and results in low power consumption. In addition, these devices can be readily integrated with complementary metal-oxide-semiconductor (CMOS) technology, and the efficiency of fabrication reduces the cost per device as well as facilitates rapid prototyping of new sensor designs. Unlike bulk acoustic wave (BAW) resonators, these devices work on a contour-mode resonator principle [418-421], in which resonator frequency is determined not by the thickness of material(s) in the resonator but by the spacing of the actuating interdigital electrodes. This allows for a variety of devices operating at various frequencies to be included on the same chip.

The magnetic field-sensing scheme in these sensors relies on the $\Delta \mathrm{E}$ effect. This is the property of magnetostrictive materials which results in an apparent change in elastic modulus (or Young's 
modulus) when magnetization states change under the influence of a magnetic field. Thin films of ferromagnetic FeGaB are used due to desirable magnetic properties. Grown by sputtering, this material exhibits relatively high $\Delta \mathrm{E}$ and magnetostriction constant, $\lambda_{s}$, at an optimized composition of $\left(\mathrm{Fe}_{80} \mathrm{Ga}_{20}\right)_{88} \mathrm{~B}_{12}$ at. $\%[378,422]$. Because it is amorphous, it is also magnetically very soft and has low anisotropy, with $\mathrm{H}_{\mathrm{c}}<100 \mu \mathrm{T}$ and $\mathrm{H}_{\mathrm{k}}<2000 \mu \mathrm{T}$. Such films (with intermittent thin spacer layers included for the sake of reducing losses from eddy currents) are grown on top of a film of piezoelectric AIN, and together the magnetic and piezoelectric phases form a laminated ME composite [423] that is mechanically coupled by strain. The AIN in turn resides on top of interdigital electrodes (IDE) that are used to transmit an AC voltage signal and to impart a contour-mode electromechanical wave to the layer stack via the $d_{31}$ mode in the AIN [424]. The layer stack and IDE together form the resonator plate that is attached to the substrate by two thin anchors but is otherwise suspended in air (Fig. 18(a)). As the resonator plate is driven in sustained contour-mode vibrations, the resonance frequency, $f_{r}$, is determined by the pitch (separation) of the IDE fingers $w_{0}$ (Fig. 18(b)), the equivalent Young's modulus of all the materials $E_{e q}$ and the equivalent density $\rho_{e q}$. As applied magnetic fields influence the elastic modulus of the magnetic material, and in turn $E_{e q}$, the output signal from the sensor changes as $f_{r}$ shifts.

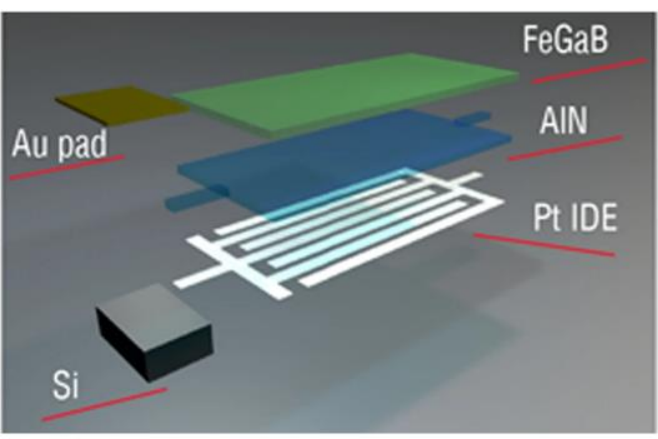

(a)

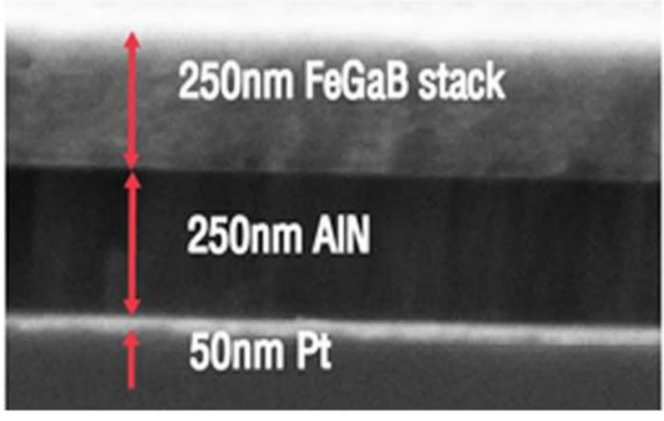

(c)

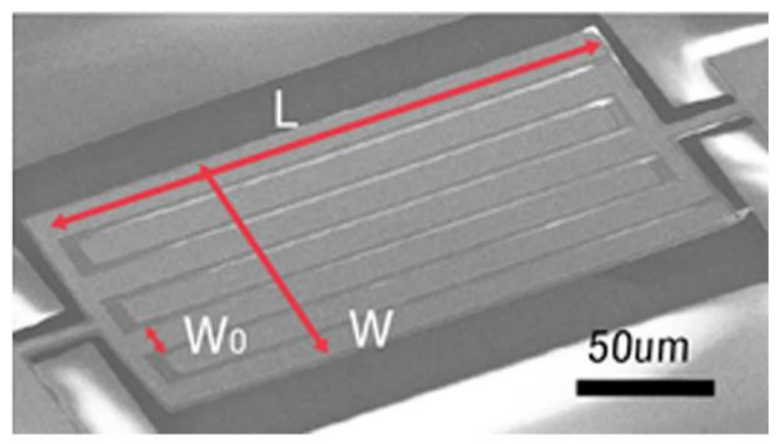

(b)

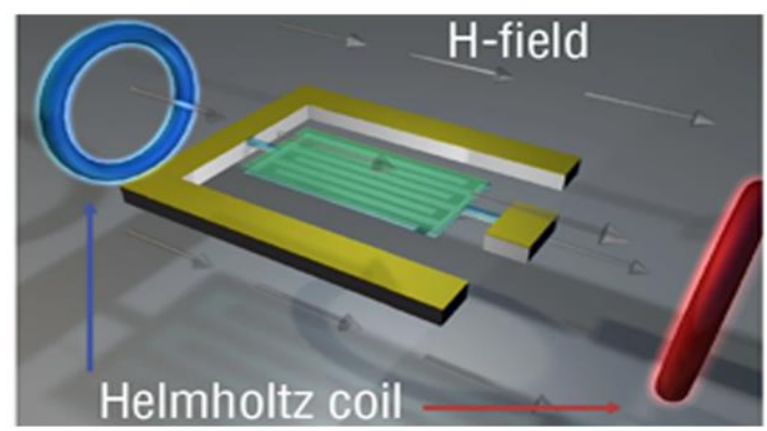

(d)

Fig. 18. (a) Schematic of the ME sensor components and materials. (b) Scanning electron microscopy (SEM) image of fabricated sensor highlighting the interdigitated electrode (IDE) geometry. (c) SEM image of ME sensor cross section. (d) Schematic of testing setup and DC magnetic field application. Replotted with permission from T. Nan et al. Scientific reports, vol. 3, p. 1985, 2013 [32]. Copyright [2013], Nature Publishing Group. 
Such a sensing mechanism is well-suited to detecting magnetic fields in a wide range of frequencies, including DC. DC and low-frequency detection are especially challenging, but is necessary in some applications such as biomedical procedures-in particular, magnetoencephalography (MEG) and magnetocardiography (MCG). Indeed, these sensors have been shown to have good detection capability with magnetic fields ranging from DC to $1.5 \mathrm{kHz}$ $[32,425-427]$.

A few characterization and signal extraction methodologies have been demonstrated, including both open-loop and closed-loop configurations. In open-loop mode, an external frequency source drives the resonator and simultaneously acts as the detector of any signal changes due to magnetic fields. This is accomplished by the one-port configuration of the electrical interface of the sensor-the way in which the probing pads are connected to the IDE. Open-loop characterization of NEMS magnetometers has been performed using a vector network analyzer and a high-frequency lock-in amplifier; in such setups, the output signal responds to changes in impedance that occur in the sensor.

The first such NEMS magnetic sensor featuring a contour-mode resonator plate had a resonance frequency $f_{r}$ of around $215 \mathrm{MHz}$ and was tested in open-loop mode using a VNA [32]. The layers featured an equal thickness of magnetic FeGaB and piezoelectric AIN of $250 \mathrm{~nm}$ each (except that the magnetic part was subdivided into 10 layers with thin $\mathrm{Al}_{2} \mathrm{O}_{3}$ spacer layers in between), as shown in Fig. 18(c). An in-situ magnetic field was applied along the width of the resonator (along $W$ in Fig. 18(b)) during sputter-deposition of the magnetic layers to pre-orient the magnetic domains. The IDE were made from Pt in order to achieve better crystal alignment of the AIN grown on top. During testing, DC magnetic fields were applied lengthwise (Fig. 18(d)). It is expected that such a process would cause magnetic domains to largely align widthwise when no magnetic field is present and then to rotate into the direction of the lengthwise magnetic field. A $90^{\circ}$ rotation of magnetic moments would maximize the $\Delta \mathrm{E}$ effect and in turn the frequency shift, thereby enhancing sensitivity.

A substantial frequency response and $Q$ factor were achieved with this device (Fig. 19). A VNA was used to drive the sensor and measure the admittance. With no magnetic field applied, a $Q$ factor of 735 and an electromechanical coupling coefficient of $1.54 \%$ were extracted by fitting the Butterworth-van Dyke model to the admittance measured in a frequency sweep (Fig. 19(a)). This value for coupling coefficient is comparable to what can be expected from similar AIN-based contour-mode resonators that do not contain magnetic layers [428]. Such frequency sweeps were then performed with incrementally increasing DC magnetic fields; the $f_{r}$ and peak admittance amplitude, $Y$, as a function of field are shown in Fig. 19(b). The $f_{r}, Y$, and $\mathrm{Q}$ all follow a trend of initially decreasing to a minimum and then increasing to a saturation value. This can be understood as a consequence of the averaged magnetization rotating until it is about halfway between lengthwise and widthwise alignment. At this orientation the magnetostriction is largest, so the material appears the most pliable, which corresponds to a frequency minimum. Likewise, damping due to activity of magnetic moments and/or domains is largest, so a minimum in $Q$ is also observed $[429,430]$. The total change in $f_{r}$ relative to the minimum is about $1 \%$, and the maximum detectivity, where $\left|\mathrm{df}_{\mathrm{r}} / \mathrm{dH}\right|$ is largest at around $500 \mu \mathrm{T}$, is roughly $1 \mathrm{~Hz} / \mathrm{nT}$. 


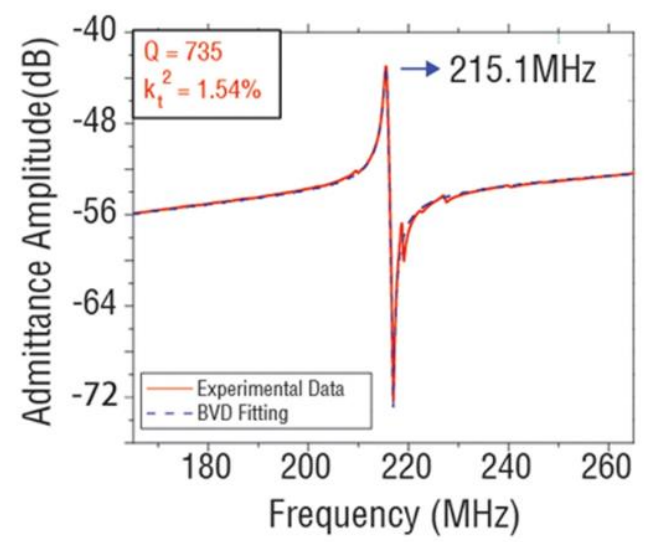

(a)

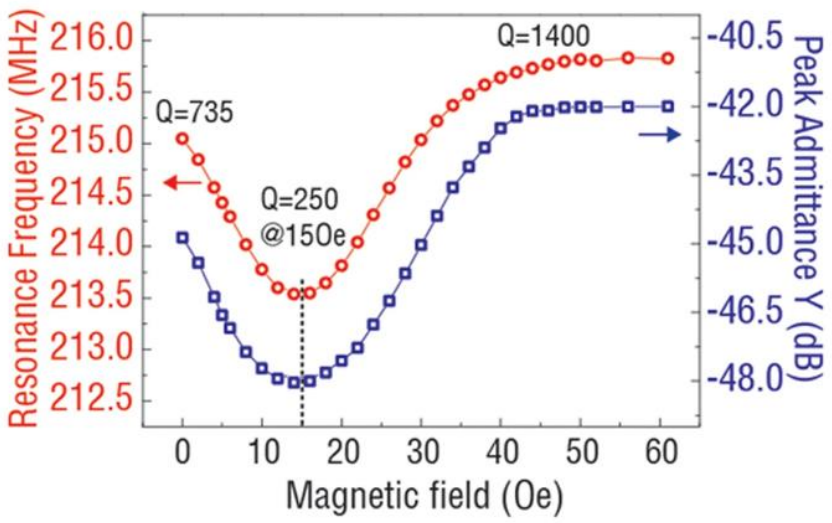

(b)

Fig. 19. (a) The admittance from the ME sensor measured by a vector network analyzer (VNA) in red, with Butterworth Van Dyke model fitting in blue. (b) ME sensor frequency shift and admittance shift with applied DC magnetic field. Reproduced with permission from T. Nan et al. Scientific reports, vol. 3, p. 1985, 2013 [32]. Copyright [2013], Nature Publishing Group.

This sensor also achieved a good MDF in an unshielded lab environment, in a test in which the applied magnetic field was changing at a constant, linear rate (Fig. 20). An additional coil was connected to a precision current source to generate a second, small magnetic field superposed on a fixed DC bias field of $500 \mu \mathrm{T}$ (to maximize sensitivity). The secondary field was reduced in magnitude until the admittance amplitude was no longer changing. With the VNA set to a power of $-12 \mathrm{dBm}$ and IF bandwidth of $200 \mathrm{~Hz}$, this method produced an MDF of about $300 \mathrm{pT}$.

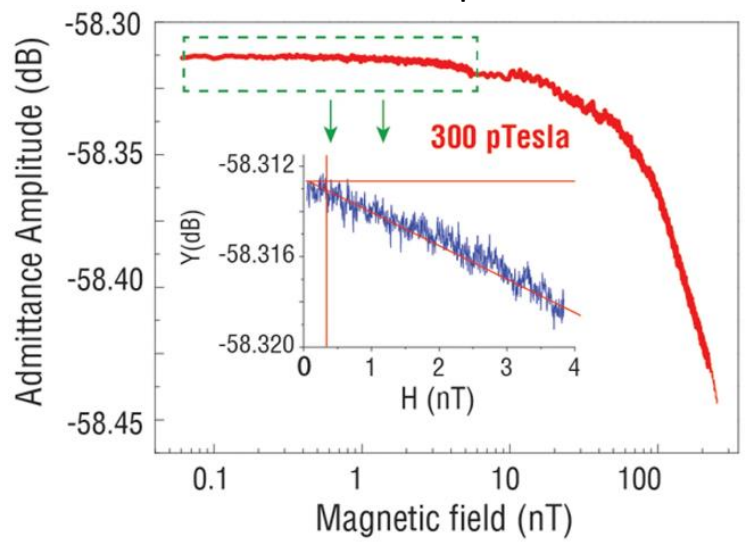

Fig. 20. MDF test, showing the change in admittance amplitude as a small, secondary DC field is reduced. This small field was superimposed on a fixed bias field of $500 \mu \mathrm{T}$, which corresponds to maximum frequency sensitivity. The inset shows a smaller range of DC field variation, from $4 \mathrm{nT}$ down to 50 pT, in which a MDF of 300 pT was obtained. Reprinted with permission from T. Nan et al. Scientific reports, vol. 3, p. 1985, 2013 [32]. Copyright [2013], Nature Publishing Group.

Another open-loop characterization methodology was demonstrated using a high-frequency lock-in amplifier [426]. In this method, a directional coupler was used to split the output AC voltage signal into a transmitted and reflected part; the transmitted signal drives the resonator, and the reflected part is wired into the input port of the lock-in amplifier. Thus, the system relies 
on monitoring the AC voltage amplitude put out by the sensor rather than the admittance. At the resonance frequency, maximum power is absorbed by the resonator, so an inverted voltage peak is observed (exemplified in Fig. 21(a)).

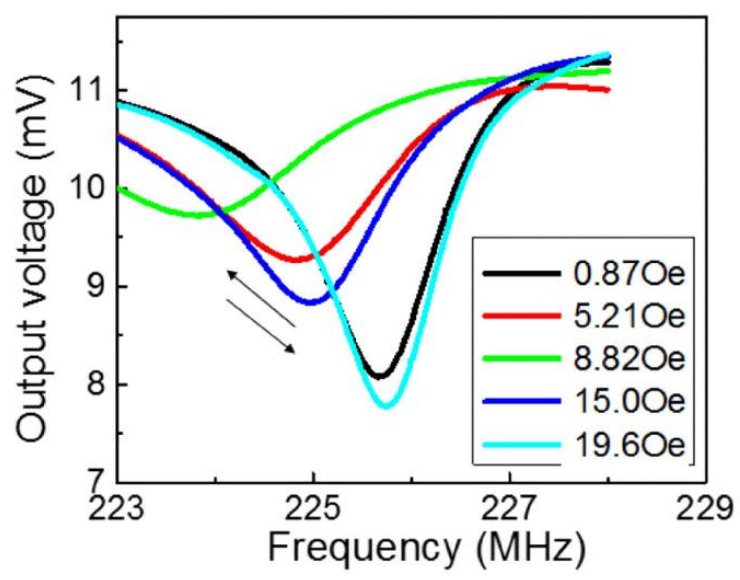

(a)

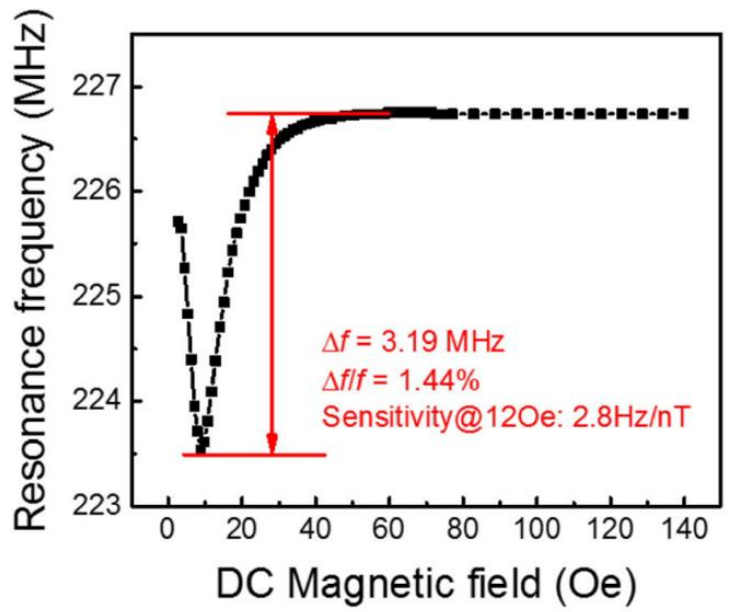

(c)

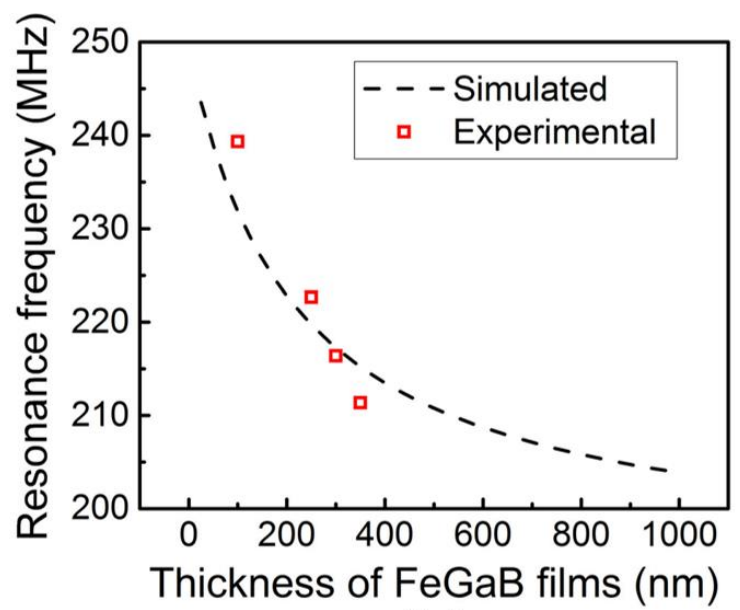

(b)

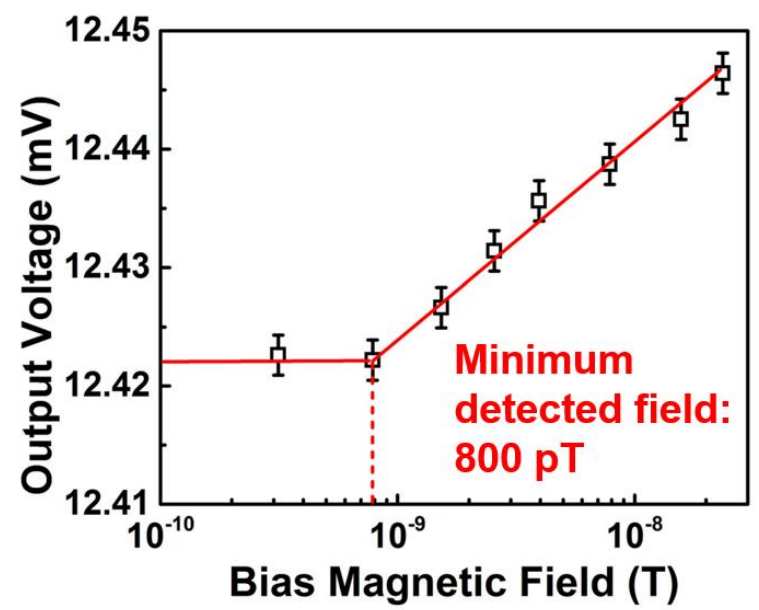

(d)

Fig. 21. (a) ME sensor voltage output as measured by a lock-in amplifier. Each frequency sweep corresponds to a different DC magnetic field. (b) Resonant frequency of resonators with different FeGaB layer thicknesses, with Eq. 1 fitted to the data. (c) Resonant frequency with applied DC magnetic field of the sensor with the highest frequency response. (d) MDF test for the same sensor, conducted with a fixed bias field of $1200 \mu \mathrm{T}$, while a secondary field was reduced in steps, yielding a MDF of 800 pT. Reproduced with permission from M. Li et al. Applied Physics Letters, 110(14), 143510, 2017 [426]. Copyright [2017], AIP Publishing.

In addition, these NEMS magnetometers were utilized to measure $\mathrm{E}$ for $\left(\mathrm{Fe}_{80} \mathrm{Ga}_{20}\right)_{88} \mathrm{~B}_{12}$ thin films, demonstrating the potential for these devices to not only advance sensor technology but to also act as test systems for more fundamental studies. Although parameters including the magnetostriction constant, $\lambda_{s}$, and the change of modulus, $\Delta \mathrm{E}$, could be measured via several techniques [378, 431-435], the baseline elastic modulus, $E$, in these thin films was not previously known, and no well-established method to measure this parameter exists. Several duplicate 
sensors were fabricated with different total thicknesses of FeGaB: 100, 250, 300, and $350 \mathrm{~nm}$ thick. With no magnetic field applied, the resonance frequency was obtained, and $f_{0}=\frac{1}{2 w_{0}} \sqrt{\frac{E_{e q}}{\rho_{e q}}}$ was fitted to the data, with $E_{e q}$ as a fitting parameter (Fig. 21(b)). The elastic modulus for the $\mathrm{FeGaB} / \mathrm{Al}_{2} \mathrm{O}_{3}$ multilayers was found to be $215 \mathrm{GPa}$.

The sensor was characterized for its frequency response and MDF via the lock-in amplifier-based technique. An improved frequency response was obtained, shown in Fig. 21 . The total frequency shift, $\Delta f_{r} / f_{r, \min }(\%)$, was $1.44 \%$, roughly $40 \%$ higher than before. And the sensitivity of 2.8 $\mathrm{Hz} / \mathrm{nT}$ was an improvement by about a factor of 3 . The MDF was then measured as follows: a small DC magnetic field was applied from a secondary coil connected to a precision current source, and this secondary field was reduced in steps, while a fixed bias field of $1200 \mu \mathrm{T}$ was maintained (Fig. 21(d)). Despite the higher sensitivity and frequency response, the obtained MDF was 800 pT, inferior to the previous result. This could be due to a lower $Q$ [430] in this sensor (not shown).

A similar NEMS sensor was also incorporated into a closed-loop, self-sustained, and low-power CMOS oscillator [425] (Fig. 22(a)). The resonator plate had a different layout, featuring 3 IDE fingers instead of the previously-used 7, and consequently a lower resonance frequency: 167.8 $\mathrm{MHz}$. In another departure from the prior design, magnetic bias during magnetic film sputtering was applied along the length of the nanoplate, inducing a magnetic anisotropy that favors a lengthwise magnetic easy axis. Tests in open-loop mode (prior to integration with the oscillator circuit) revealed a higher mechanical quality factor, $Q_{m}$, of 1084 and an electromechanical coupling coefficient, $k_{t}{ }^{2}$, of $1.18 \%$ (Fig. 22(b)). This gives a figure of merit (FOM) of $\sim 13$ (FOM = $k_{t}{ }^{2} \bullet Q$ ), which is comparable to conventional resonators with the same AIN thickness [436]. The frequency response to DC magnetic fields was characterized by fields applied both widthwise and lengthwise to the resonator, and the results indicate an anisotropic response due to the induced magnetic anisotropy in the FeGaB layers (Fig. 22(c)). The characteristic trend associated with the $\Delta \mathrm{E}$ effect-in which $f_{r}$ first declines to a minimum and then reaches a maximum at magnetic saturation [377] - now occurs with magnetic fields applied across the width of the nanoplate (as a result of the bias during growth of FeGaB). However, the anisotropy was rather large, so that $10 \mathrm{mT}$ was required to cancel the anisotropy field; this has the undesired effect of reducing the maximum frequency sensitivity, $\left|\mathrm{df}_{\mathrm{r}} / \mathrm{dH}\right|$, which in turn is likely to impair the MDF capability in the sensor.

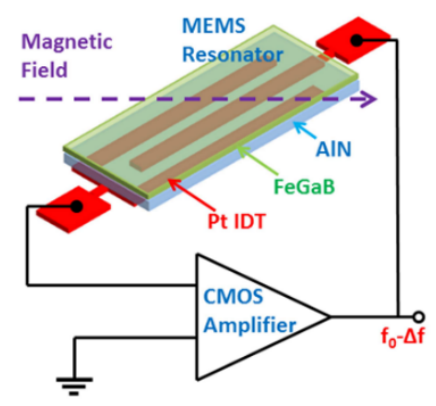

(a)

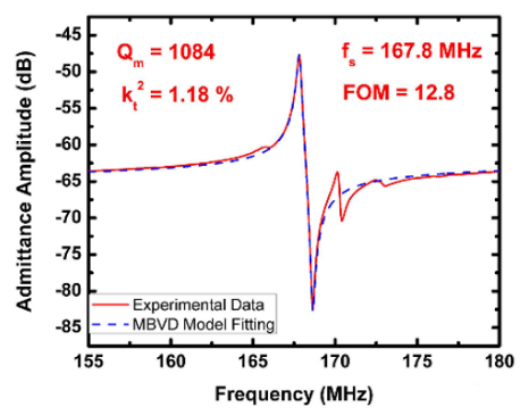

(b)

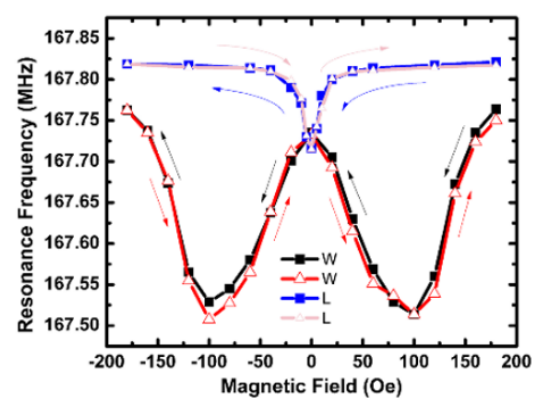

(c) 
Fig. 22. (a) Schematic of ME sensor nanoplate resonator integrated with a self-sustained CMOS oscillator. (b) Open-loop measurement of ME sensor admittance in red and Modified Butterworth Van Dyke model fitting in blue, from which parameters of the resonant frequency (with no field applied), Q, and $k_{t}{ }^{2}$ are extracted. (c) Resonant frequency response to DC magnetic field applied along the width and length of the resonator. Reproduced with permission from $\mathrm{Y}$. Hui et al. Journal of Microelectromechanical Systems, 24(1), 134-143, 2014 [425]. Copyright [2014], IEEE.

The contrast in sensor response to different field directions can be taken advantage of for electronic compass applications; however, a limitation to be considered is performance variation with temperature. In particular, the variation of $f_{r}$ with temperature was measured, and a nonlinear temperature coefficient of frequency (TCF) was recorded from -67 to $-156 \mathrm{ppm} / \mathrm{K}$, which is larger than typical TCF of AIN-based resonators due to the additional temperature coefficient of the FeGaB film. This challenge can be overcome by integrating a temperature sensor that is unaffected by magnetic fields, such as a conventional AIN resonator without magnetic films, to be used for temperature calibration [437].

After initial open-loop testing, the nanoplate resonator was then wire-bonded to a CMOS IC chip (Fig. 23(a)) to make the first demonstration of a self-biased magnetometer based on a ME MEMSCMOS oscillator. The circuit scheme formed a Pierce oscillator by way of three transistors arranged to constitute a CMOS inverter and a bias in its active region. The integrated sensor was characterized in terms of its frequency output (Fig. 23(b)), phase noise and Allan deviation, as well as repeat tests of frequency response and a demonstration of the device used as a compass. Repeated tests of frequency response (Fig. 23(c) and Fig. 23(d)) showed that the $f_{r}(H)$ curve had changed. This indicates that the magnetization states had somehow changed after the initial round of testing in open-loop mode had been completed, but further verification of this phenomenon is needed. 


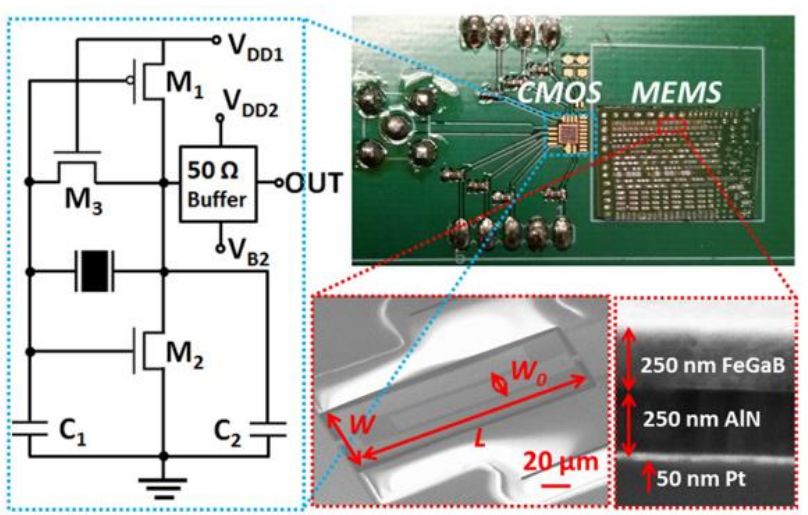

(a)

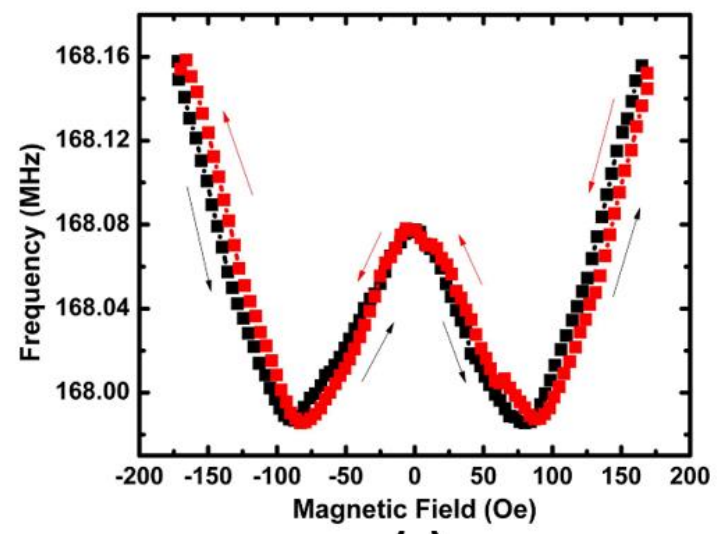

(c)

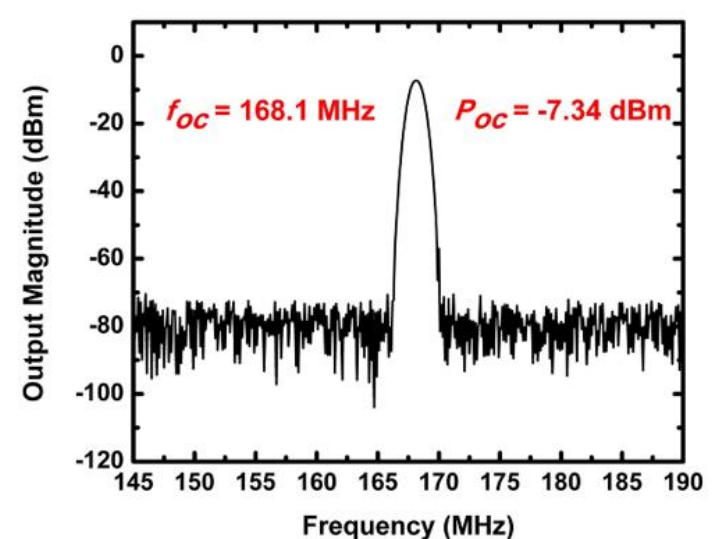

(b)

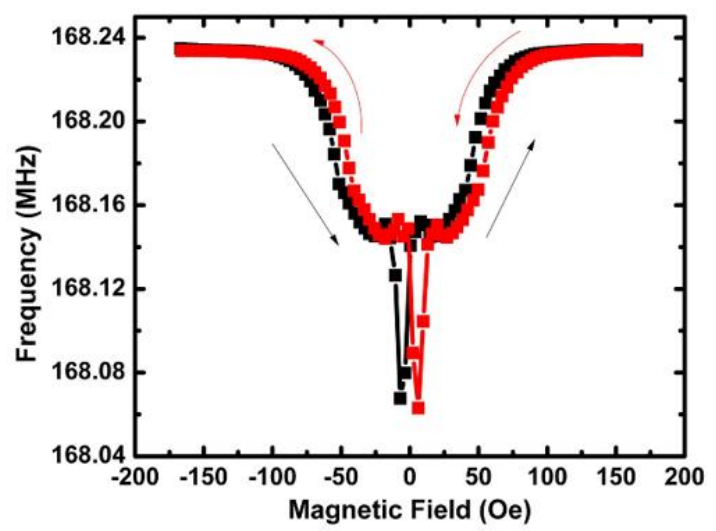

(d)

Fig. 23. (a) Integration of the ME sensor chip with the CMOS integrated circuit (IC) chip on a custom-designed circuit board. The circuit schematic shows the oscillator design, with $C_{1}=C_{2}=$ $1.5 \mathrm{pF},(\mathrm{W} / \mathrm{L})_{\mathrm{M} 1}=300,(\mathrm{~W} / \mathrm{L})_{\mathrm{M} 2}=675,(\mathrm{~W} / \mathrm{L})_{\mathrm{M} 3}=0.03$. (b) Measured frequency output spectrum in closed-loop mode. (c) Resonant frequency response of AIN/FeGaB MEMS-CMOS oscillator to a DC magnetic field sweep applied along the width. (d) Frequency response of the oscillator to a DC magnetic field sweep applied along the resonator length. Reproduced with permission from Y. Hui et al. Journal of Microelectromechanical Systems, 24(1), 134-143, 2014 [425]. Copyright [2014], IEEE.

The integrated MEMS-CMOS oscillator showed maximum sensitivity with the DC field applied lengthwise to the nanoplate, and-crucially-featured a linear response in the vicinity of zero field, which enables the sensor to operate without a bias field, or in other words, to be self-biased. A sensitivity of $169 \mathrm{~Hz} / \mu \mathrm{T}$ was obtained (Fig. 24), and, together with results of the Allan deviation measurement, an estimate for detectivity was calculated to be $16 \mathrm{nT} / \mathrm{Hz}^{1 / 2}$. The sensor was then used in a simulated real-world application by demonstrating its functionality as a compass in a field approximating the Earth's magnetic field. A $50 \mu \mathrm{T}$ field was applied at different angles with respect to the width of the nanoplate resonator, and a frequency shift strongly dependent on angle was observed, due to the strongly anisotropic sensitivity of the AIN/FeGaB resonator. The angular resolution was determined to be $0.34^{\circ}$ in the range of $0^{\circ}$ to $180^{\circ}$ and $0.54^{\circ}$ for the $180^{\circ}$ to $360^{\circ}$ range. 


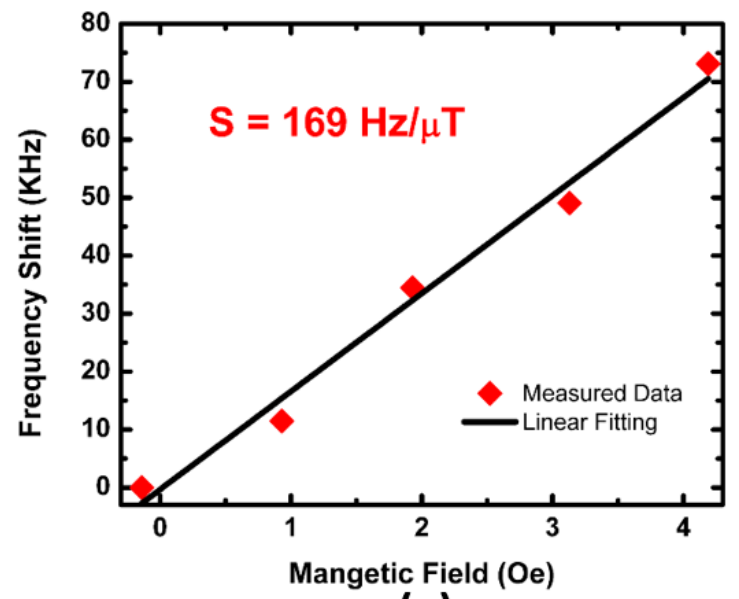

(a)

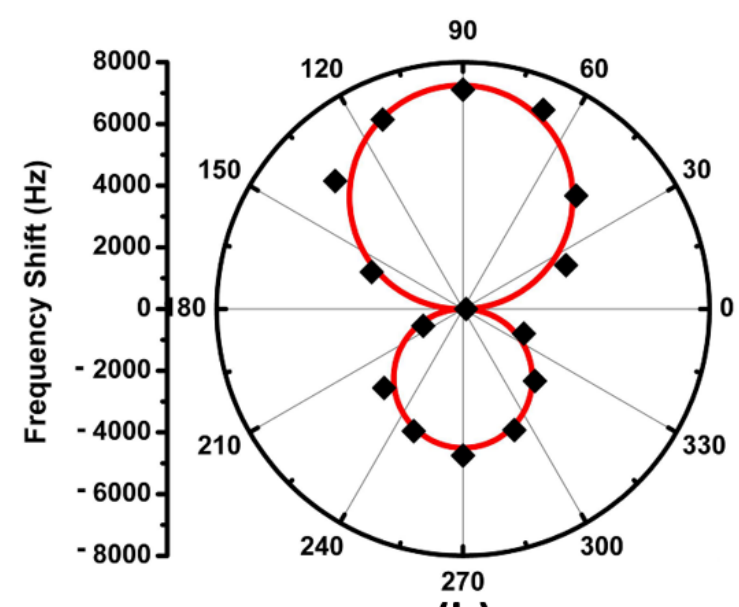

(b)

Fig. 24. (a) Frequency sensitivity of the MEMS-CMOS oscillator to magnetic field applied along the length of the resonator with zero bias. (b) Angular dependence of frequency shift in the oscillator, demonstrating its capability as an electronic compass. Reproduced with permission from Y. Hui et al. Journal of Microelectromechanical Systems, 24(1), 134-143, 2014 [425]. Copyright [2014], IEEE.

There is a wide array of fundamental questions as well as engineering challenges about such NEMS/MEMS ME magnetometers that remain to be explored. Firstly, it is critical to better understand the magnetic domains within the magnetic layers and the major properties that determine the $\Delta \mathrm{E}$ effect, including the effective anisotropy energy density and the magnetic easy axis alignment. Because of the microscopic scale of the resonator plate, and the growth of the ME thin films on top of electrodes, the films may be sensitive to effects that are less relevant for thin films grown on a much thicker substrate. For example, whereas the stress distribution in a thin film on top of a much thicker substrate may be approximately uniform [438], in a thin film system like the resonator plate, a stress gradient is expected $[439,440]$. In addition, patterned structures, like the IDE, affect stress when an AIN thin film is grown on top [441]. To shed more light on such fundamental aspects of the sensors, we have been systematically investigating the parameters that influence sensor performance, with particular focus on how the frequency response can be optimized.

Beyond that, possible future investigations include the use of new materials, new resonator plate designs, and new fabrication processes. One benefit worth exploring is that these initiatives may help to raise the $Q$ of the resonator; from a practical standpoint, this is expected to improve the MDF of the magnetometer. For example, we have completed simulations that indicate less scattering of vibration energy would result for certain resonator shapes. As for materials, contour-mode resonators made with ScAIN were recently demonstrated and showed improved $\mathrm{Q}$ and $k_{t}{ }^{2}$ compared to AIN [442]. New fabrication processes could also yield benefits such as achieving flat material layers and the freedom to use more conductive (or lighter) materials for the IDE, if the layer sequence were inverted. In summary, a multitude of opportunities remain to continue researching these ME magnetometers, either from a fundamental angle of looking at 
the material science involved, or from an application-focused perspective of optimizing performance.

\subsection{Magnetoelectric random-access memories (ME-RAMs)}

Over decades, the progress in digital technologies has relied on transistor downscaling in order to enhance the operation speed and increase the amount of electronic data that can be stored. At the same time, the size of bits has been reduced concurrently with the electronics (i.e., patterned media), to significantly increase the areal density of stored information in, e.g., har disk drives (HDDs). At present, when chips can be prepared on the scale of hundreds of nanometers (smaller than many viruses), and the size of a single magnetic bit can reach sub-10 $\mathrm{nm}$, magnetic devices face another rather fundament challenge: the overheating due to the electric currents involved during information writing [177].

Magnetization switching in devices is conventionally accomplished by localized magnetic fields (generated via electromagnetic induction) or by spin-polarized electric currents (spin-transfer torque) [443]. Both principles require relatively high electric currents and thus involve significant loss of energy in the form of heat (by Joule effect). The currents needed to operate conventional magnetoresistive random-access memories (MRAMs) are of the order of $10 \mathrm{~mA}$, whereas the spin-transfer torque variant of MRAMs requires currents of at least $0.5 \mathrm{~mA}$. This is still a factor five times higher than the currents delivered by highly miniaturized metal-oxide-semiconductor field-effect transistors [444]. Other types of non-volatile memories (such as NOR or NAND flash) integrated, for example, in 'universal series bus' (USB) drives, digital cameras, mobile phones, synthesizers, game consoles, robotics or medical electronics, also require of considerably high currents and are, therefore, not optimized from an energetic point of view. This makes refrigerating systems indispensable to avoid temperature rises, resulting in an extra source of energy consumption. In addition, excessive heating in computers and data servers can damage the memories and induce loss of the recorded information. For these reasons, data center providers work intensively to find strategies to face this problem, using sophisticated and costly cooling systems or directly locating their servers under the ocean or in cold countries.

The energy needed to switch the magnetization of a magnetic object is, in a first approximation, proportional to its coercivity. Application of voltage has been shown to reduce $\mathrm{H}_{\mathrm{c}}$ in various types of magnetic materials (see e.g., Table IV). Thus, the use of voltage can lead to significant energy saving with an important cost reduction (ultra-low power ME devices). Table $\mathrm{V}$ lists the energy needed to write a single bit of information in the different types of existing memories $[445,446]$. Looking at the Table, volatile SRAM and DRAM memories seem to be amongst the most energy efficient. However, because of their volatility, these memories need a constant power in order to retain data and require periodical refreshments, which impose a continuous energy consumption. During the last few years, voltage (or electric field) control of magnetism has emerged as a fundamentally different approach towards the implementation of ultralow-heatdissipation memories operated at the nanoscale. Very recent theoretical studies predict that the energy needed to write a magnetic bit using voltage could be as low as $10^{-6} \mathrm{pJ}$ [75]. Since voltage is applied through an electrically insulating layer (with an infinite resistance), Joule heating effects 
are nearly zero. ME approaches have been realized in numerous classes of materials and architectures where an efficient modulation of various parameters, such as coercivity, magnetic moment, magnetic anisotropy, remanent magnetization, exchange bias or topological spin structures, has been achieved $[9,447]$. All these effects can be employed for the realization of ME random-access memories (ME-RAMs), from which various prototypes have been demonstrated. Experimentally, for memory cells with lateral size of around $20 \mathrm{~nm}$, depending on the underlying mechanism responsible for the observed $\mathrm{ME}$ effects, energy dissipation per switching bit event can be as low as $\sim 10^{-6} \mathrm{pJ}$ (for strain-mediated ME coupling) or $10^{-3} \mathrm{pJ}$ (for charge mediated coupling) [447]. In ME-RAM memories based on reorientation of the magnetic easy axis (voltage-controlled anisotropy ME-RAMs), switching events involving interfacial oxygen have been demonstrated with a writing energy of $10^{-3} \mathrm{pJ} / \mathrm{bit}$ [448]. As evidenced in Table 1 , these energies represent several orders of magnitude lower than those required to write data bits in other types of non-volatile memories.

Table V: Consumed energy to write one bit for the different main types of existing memory systems (volatile in light blue and non-volatile in light pink) [75, 445-448]. For HDD, the energy dissipated by rotation of the disk and the movement of the read/write arm is also included.

\begin{tabular}{ccc}
\hline $\begin{array}{c}\text { Memory Scheme } \\
\text { (V: volatile / } \\
\text { NV: non-volatile) }\end{array}$ & Acronym & $\begin{array}{c}\text { Writing } \\
\text { energy/bit } \\
\text { (pJ) }\end{array}$ \\
\hline Dynamic (V) & DRAM & $10^{-2}$ \\
Static (V) & SRAM & $10^{-3}$ \\
Memristor (NV) & RRAM & $10^{-1}$ \\
Toggle Magnetoresistance & MRAM & 1 \\
Spin transfer torque & STT-RAM & $10^{-1}$ \\
Phase Change (NV) & PC-RAM & 10 \\
Ferroelectric (NV) & FE-RAM & $10^{-2}$ \\
Flash (NV) & NOR & $10^{2}$ \\
Hard-disk drive (NV) & HDD & $>10^{3}$ \\
Magnetoelectric (NV) & ME-RAM & $\begin{array}{c}\mathbf{1 0} \\
\text { (theoretical) } \\
<10^{-3}(\text { expt.) }\end{array}$
\end{tabular}

MRAM technology is based on spintronics (spin-based electronics), incorporating the spin (i.e., magnetism) as an additional degree of freedom to electron charge. Conventional MRAMs 
combine an ensemble of magnetic storage units (the so-called magnetic tunnel junctions, MTJ) with standard CMOS transistors, rendering non-volatility of the stored information, ultra-fast processing rates and high read/write endurance [449]. Each MTJ comprises a fixed ferromagnetic layer (typically pinned via the direct exchange coupling with an antiferromagnet), a thin dielectric tunnel barrier and a soft ferromagnetic layer (free layer), whose orientation can be changed by applying a magnetic field or, as demonstrated in recent years, a spin-polarized electric current (spin-transfer torque effect). The two states will be read through the changes in magnetoresistance between the " 1 " and " 0 " configurations. When a current ( $\left.i_{\text {read }}\right)$ will traverse the MTJ, the resistance of each unit will depend on the relative orientation between the free and the pinned FM layers. During the reading stage, the magnetoresistance is low (or high) depending on whether the two layers are parallel (or antiparallel) to each other. While reading the information stored in each MTJ of the MRAM is rather simple, the writing process is more complicated and remains not optimized. Originally, the bit writing relied on two orthogonal magnetic fields generated by electric current flowing in the so-called "write or word lines". However, relatively high writing currents, of the order of $10 \mathrm{~mA}$, were needed. This was not convenient in terms of energy efficiency and also because the high currents induced undesirable switching in neighboring MTJs, particularly as MRAMs were progressively shrunk in size. Several alternatives have been proposed to overcome this issue, such as thermally-assisted switching (which works in an analogous way as thermally-assisted magnetic recording) $[444,450]$ or spintransfer MRAMs, in which the switching of the free layer is controlled by a current of spinpolarized electrons that traverses the MTJs [451]. However, in both cases, the power consumption remains exceedingly high and the requirement for moderate electric currents also precludes the reduction in size of the associated transistors, thus hindering the miniaturization of MRAMs. To improve energy efficiency, several ME-RAM prototypes have been proposed, where either the orientation of the free layer is controlled with voltage (as described below), or the exchange bias field in the pinned layer is tailored with an electric field. In most ME-RAMs designs, the MTJs comprise either a FE spacer [452, 453], a multiferroic (FE+AFM) layer [452, 454, 455] or a thin free FM layer that gets reoriented by the FE [456-460]. 

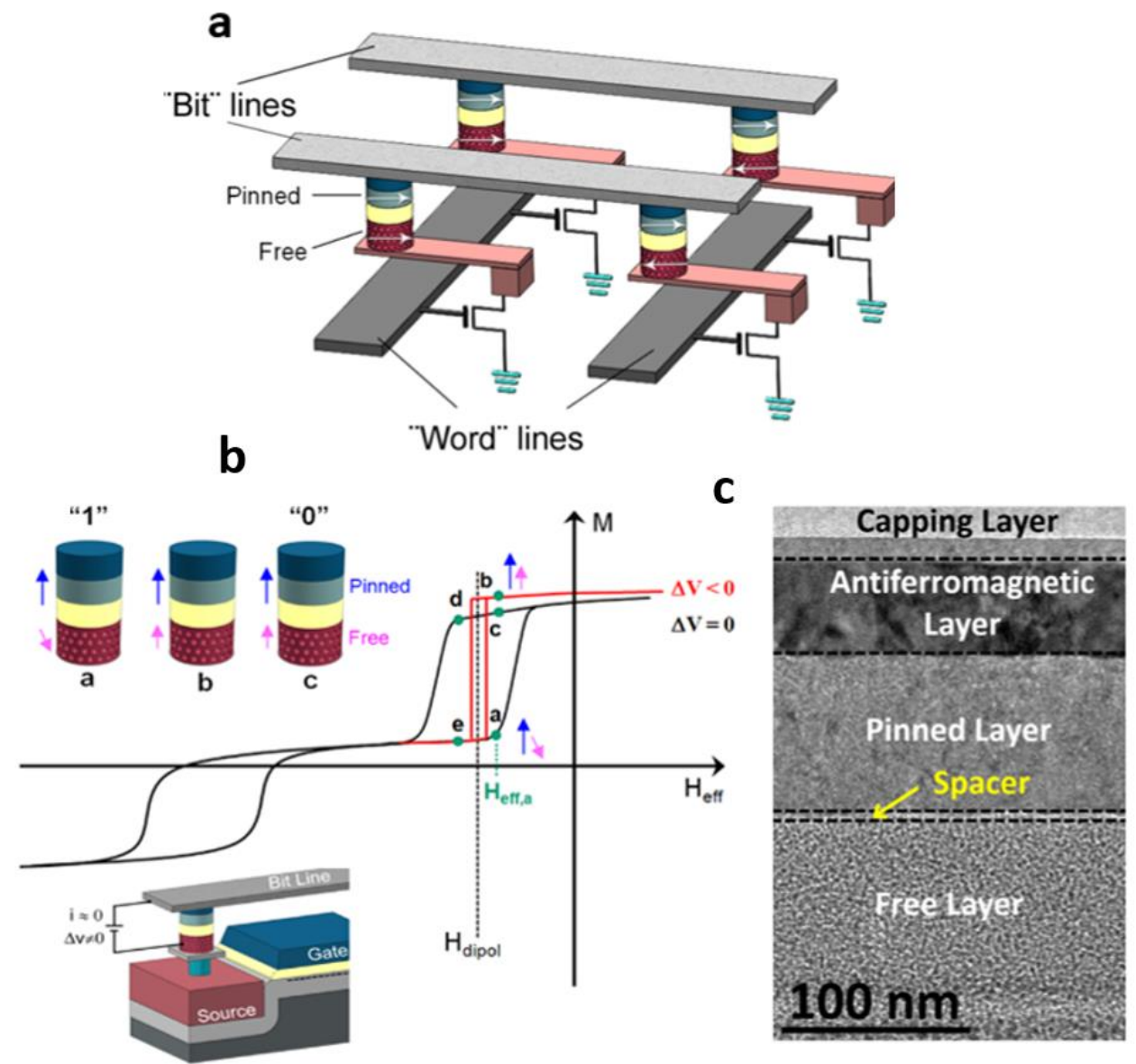

Fig. 25: (a) Schematic drawing of an ME-RAM device in which each magnetic unit consists of a magnetic tunnel junction (MTJ) coupled to transistor. (b) Operating principle of the ME-RAM based on voltage-controlled magnetic anisotropy; the sub-loop at the bottom-left corresponds to the reversal of the pinned layer, whereas the one on the upper right corresponds to the reversal of the free layer. In black are the loops in absence of external electric field and in red is the sub-loop of the free layer upon application of a negative voltage. (c) High-resolution transmission electron microscopy image of an archetypical multi-layer structure of a MTJ.

Let us describe, as example, the operation principle of an ME-RAM with voltage-controlled anisotropy in the free layer. The basic idea is to grow a soft magnetic free-layer with no clearly defined magnetic easy axis, where voltage-tunable perpendicular anisotropy competes with inplane magnetic shape anisotropy. Application of voltage reduces the perpendicular anisotropy so that the magnetic stray field emanated by the pinned layer orients the free layer either parallel or antiparallel to its orientation (depending on the initial configuration of the two layers), to give rise to a decrease (or increase) of the magnetoresistance. Crossbar arrays (analogous to those utilized in neuro-morphic computing) are fabricated. To illustrate the switching process, let us consider a MRAM operating along the perpendicular-to-plane direction. First, the MTJ is resting in the high resistance configuration " $a$ " in Fig. 25(b), corresponding to an effective magnetic field $\mathrm{H}_{\text {eff,a. }}$. The magnetoresistance is high (state "1") because the two layers are essentially antiparallel 
to each other. When a negative electric field is applied, $\mathrm{H}_{\mathrm{c}}$ in the free layer decreases. As a consequence, the free layer is forced to relax to state " $b$ " for the given field $\mathrm{H}_{\text {eff,a. Once voltage }}$ is removed, the two layers remain parallel to each other since no external magnetic field is applied to vary $\mathrm{H}_{\text {eff }}$ (state " $\mathrm{c}$ "). Hence, keeping constant the $\mathrm{H}_{\text {eff, a }}$ value, the tunnel junction will change from a high resistance state " 1 " to a low resistance state " 0 " upon voltage application. Analogously, the magnetic state could be reversed from " 0 " to " 1 " by applying the same reasoning from configuration " $d$ " to " $e$ ".

Remarkably, in the long-term, the use of ME materials could be also relevant in the search for alternatives to current computing methods. So far, computers rely on Von Neumann's architecture in which data processing and storing take place in different sub-devices (i.e., central processing unit and memory, respectively) bridged by the communication bus. This is not only detrimental to data processing speed but also to energy efficiency, making the search for new computing configurations fundamental for information technology. Neuromorphic computing relying on the use of devices that emulate the electrical behavior of the biological synapse (i.e., brain-inspired computing), which is the memory and learning element of the brain, has emerged as an alternative, envisaging simultaneous low-power information storage/processing. The magneto-ionic approach, which ensures remarkable energy-efficient and robust tuning of the magnetic properties, might be a firm candidate to mimic synapse's way of functioning [461].

\subsection{Other Devices}

\subsubsection{Tunable Inductors}

As one of the basic passive components in electronic circuits, different design approaches for inductors for RF communication systems have been developed in the past few decades. Owing to the simple fabrication process and high inductance density, magnetic inductors are the frequently-used method among them. For magnetic inductor applications in the RF domain, there is a tradeoff between high inductance density, Q-factor and the frequency extension due to lower relative permeability under higher ferromagnetic resonance (FMR) frequency. Inductors with tunable inductance and frequency are always desired for achieving reconfigurable electronics. Here, the tunable inductor allows for changing inductance and working frequency under an external controlling signal, such as magnetic field, DC voltage, etc. There have been various techniques designed to realize tunability for passive MEMS inductors: changing the turns of coil by using switches [462]; applying DC current bias [463]; ME coupling [35, 36]; etc. Nevertheless, none of these tuning approaches is perfect and a tradeoff has to be made according to practical applications. Our work has been focused on achieving high inductance tunability and high Q-factor by utilizing the strong inverse ME coupling in ME heterostructures. The inductor can be easily tuned by applying a magnetic field, which is due to the change of magnetic anisotropy. For electric field tuning, the magnetic permeability is controlled by the strain-mediated ME coupling, which is a combination of piezoelectric and magnetostrictive effects. A high peak Q-factor of 32.7 and a constant inductance of $1.4 \mathrm{nH}$ at the operation frequency of $1 \mathrm{GHz}$ were realized by Chen et al. [36] By exploiting the ME coupling between 
magnetostrictive iron gallium boron ( $\mathrm{FeGaB}$ ) multilayers and a lead magnesium niobate titanate (PMN-PT) piezoelectric slab, a high inductance tunability of $69.2 \%$ and $191 \%$ were demonstrated under magnetic and electric field tuning, respectively.

As shown in Fig. 26(a), a summary of Q-factor and inductance density of the state-of-the-art magnetic inductors with various structure (spiral, solenoid, stripline and meander) and operating frequencies is presented. Compared to other structures, the spiral and solenoid inductors are more compact and have higher inductance density. However, the complex fabrication process and parasitic capacitance need to be considered for the tradeoff. One example of the tunable solenoid inductor is presented below. The SEM top view and 3D structure of the integrated inductor based on ME coupling with 3.5 turns are shown in Fig. 26(b) and (c). Higher efficiency can be achieved by confining the magnetic flux inside the coils along the length direction. Due to the benefits of $\mathrm{FeGaB} / \mathrm{Al}_{2} \mathrm{O}_{3}$ multilayer, such as large magnetostriction constant, high self-biased FMR frequency, low eddy current loss, etc. [378, 379], high Q-factor inductors were obtained. A magnetic field along the length direction of the inductor was applied for testing its magnetic field tunability. The inductance and Q-factor as a function of frequency under different applied magnetic field were measured and displayed in Fig. 27(a) and (b). The inductance remains almost unchanged over a wide frequency range from $\mathrm{DC}$ to $3 \mathrm{GHz}$. Upon increasing the bias magnetic field from 0 to $50 \mathrm{mT}$, the inductance decreases continuously, and a maximum tunability of $69.2 \%$ is achieved due to the decreases in the relative permeability of the magnetic multilayer. Opposite to the change in inductance, the Q-factor increases by $67.9 \%$ because of the decrease in eddy current loss. As shown in Fig. 27(c) and (d), for voltage tuning, the inductance increases from 1.2 to $3.5 \mathrm{nH}$ by applying an out-of-plane electric field from 0 to $10 \mathrm{kV} / \mathrm{cm}$. The Q-factor also increases from 0.5 to $2 \mathrm{GHz}$, and the highest inductance tunability of $191 \%$ is obtained at $1.5 \mathrm{GHz}$. Compared with other inductor designs, this design has a continuous and large inductance tunability. Resulting from the simple device structure, its long-term performance is also stable. However, the main shortcoming of low Q-factor limits the application of this inductor structure. 


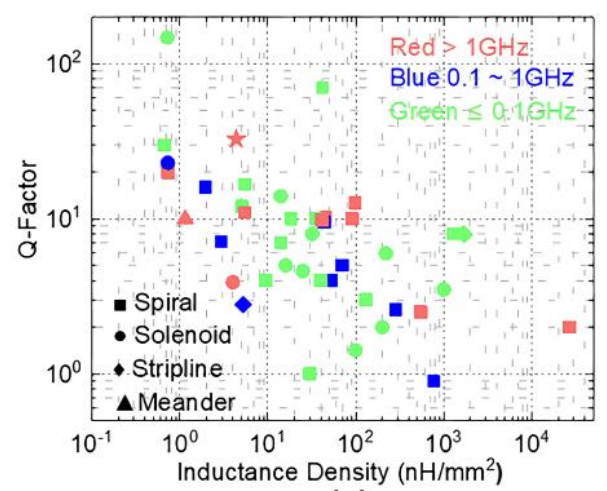

(a)

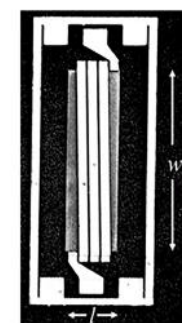

(b)

(c)

Fig. 26. (a) Q-factor and inductance density of the state-of-the-art magnetic inductors with various structure and operating frequency. (b) SEM top view and (c) schematic of the integrated RF MEMS inductor. Reprinted with permission from $\mathrm{H}$. Chen et al. IEEE Transactions on Microwave Theory and Techniques, 68(3), 951-963, 2020 [36]. Copyright [2020], IEEE. 


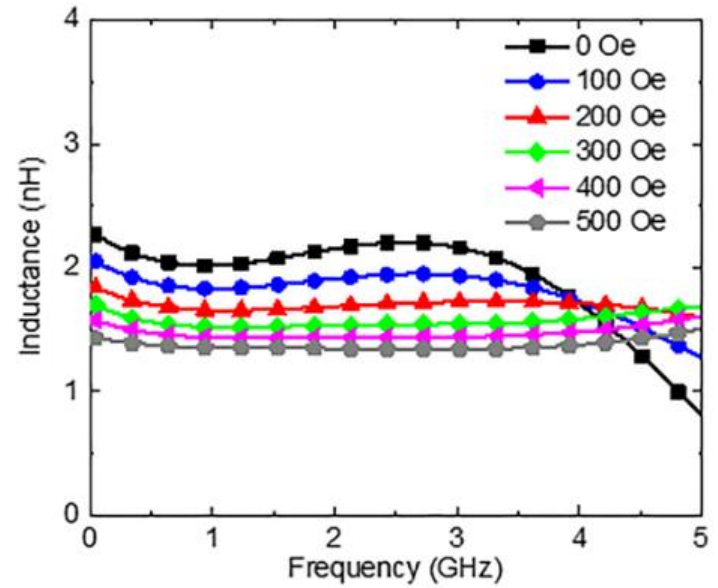

(a)

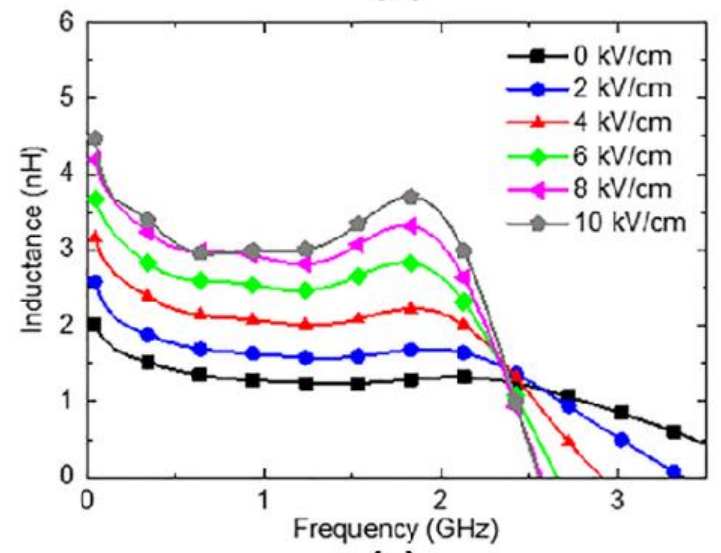

(c)

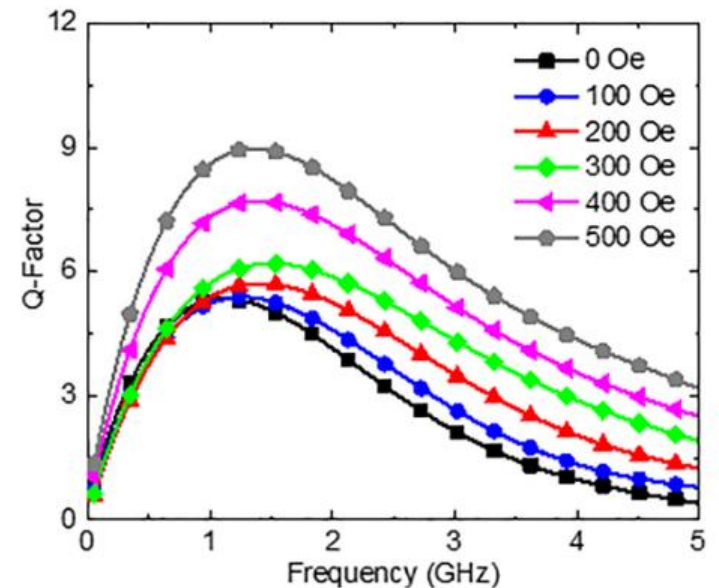

(b)

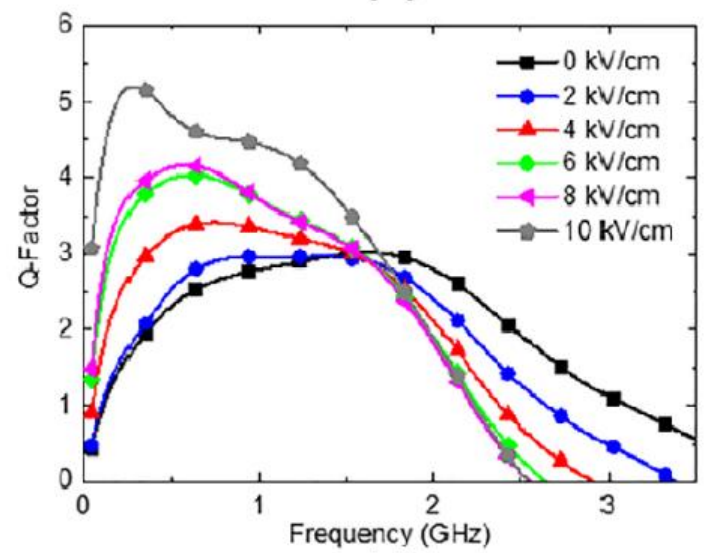

(d)

Fig. 27. Tunability of the solenoid inductor. (a) Inductance and (b) Q-factor of the tunable inductor under magnetic field from 0 to $50 \mathrm{mT}$. (c) Inductance and (d) Q-factor of the tunable inductor under electric field from 0 to $10 \mathrm{kV} / \mathrm{cm}$. Reproduced with permission from $\mathrm{H}$. Chen et al. IEEE Transactions on Microwave Theory and Techniques, 68(3), 951-963, 2020 [36]. Copyright [2020], IEEE.

\subsubsection{Tunable Filters}

Filters have been widely used in electronic systems for removing unwanted signals. Ultra-wide band (UWB) and low-loss filters with dual magnetic and electric field tunability are highly desired in modern electronic systems such as reconfigurable and multiband communication systems. One of the most widely used tunable filters is the yttrium-iron-garnet (YIG) magnetic resonator, which has a high Q-factor and multi-octave bandwidths [464-466]. Compared to tunable magnetic filters, electrostatically tunable filters are more lightweight, compact and power efficient. In addition, such ME tunable filters provide more design flexibility for both electric and magnetic field tunability. Another competitive design technique for achieving low loss and lowpower-consuming tunable filters is based on MEMS varactors and switches [467, 468]. By utilizing the non-reciprocal performance of magnetostatic surface wave (MSSW) in magnetic films, Lin et al. demonstrated the first non-reciprocal MEMS bandpass filter with dual $\mathrm{H}$ - and E-field tunability 
[39]. The filter is designed with an inverted-S-shaped structure, which is coupled with a rotated NiZn ferrite film. The optical image and schematic of the fabricated filter are shown in Fig. 28(a) and (b), respectively. The magnetic phase NiZn ferrite slab is deposited by a spin spray method that is fast and low cost. After that, the backside of the $\mathrm{Si}$ substrate is removed by deep reactive ion etching (DRIE) technique and glued to a (011) cut PMN-PT slab to form an ME heterostructure. The magnetic field tunability of the bandpass ME filter is demonstrated by measuring $S$ parameters and shown in Fig. 28(c) and (d). By increasing the DC bias field from $10 \mathrm{mT}$ to $40 \mathrm{mT}$, the resonance frequency is tuned from $3.78 \mathrm{GHz}$ to $5.27 \mathrm{GHz}$, which indicates a frequency tunability of $0.5 \mathrm{GHz} / 10 \mathrm{mT}$. All the reflection coefficients $\mathrm{S}_{11}$ are below $-20 \mathrm{~dB}$, therefore, most of the energy is absorbed by the NiZn ferrite film. After bonding to the PMN-PT slab, the voltage-tunable behavior of $S_{11}$ and $S_{21}$ are measured and presented in Fig. 28(e) and (f). As the central frequency increases from $2.075 \mathrm{GHz}$ to $2.295 \mathrm{GHz}$ under an electric field of $4 \mathrm{kV} / \mathrm{cm}$, a frequency tunability of $55 \mathrm{MHz} /(\mathrm{kV} / \mathrm{cm})$ is achieved.

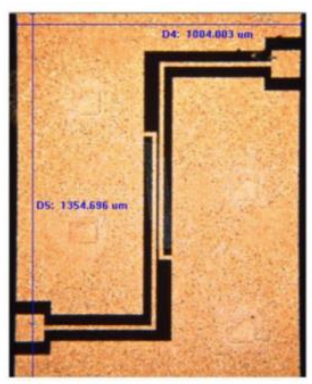

(a)

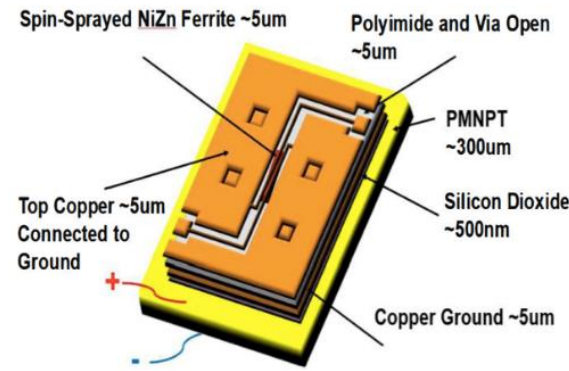

(b)

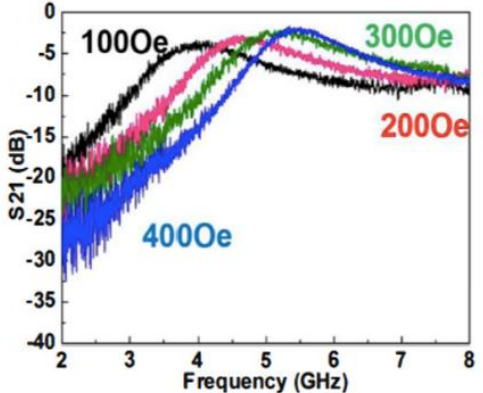

(c)

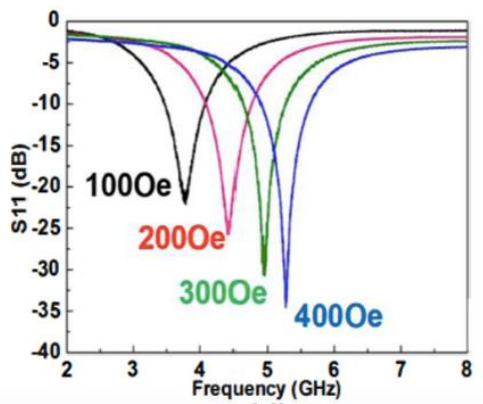

(d)

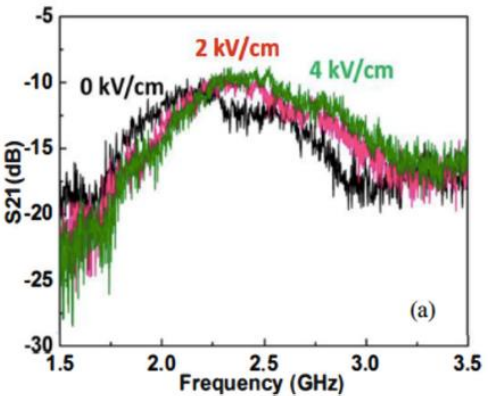

(e)

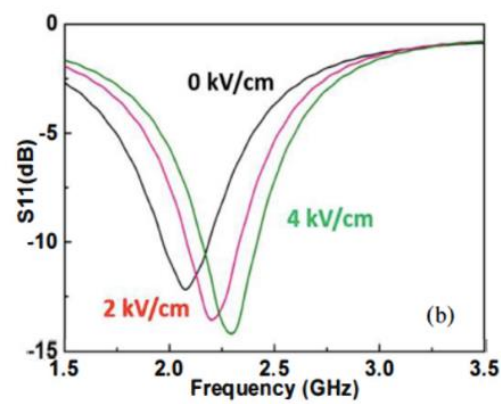

(f)

Fig. 28. (a) Optical and (b) schematic of the integrated ME bandpass filter. (c) $S_{21}$ and (d) $S_{11}$ of the filter tuned by $\mathrm{H}$-field. (e) $\mathrm{S}_{21}$ and ( $\mathrm{f}$ ) $\mathrm{S}_{11}$ of the filter tuned by E-field. Reproduced with permission from H. Lin et al. IEEE MTT-S International Microwave Symposium, 2015 [39]. Copyright [2015], IEEE.

More recently, a tunable RF bandpass filter based on MEMS ME resonators with contour mode of transmission was reported [40]. The phase locking between two coupled ring-shaped resonators composed of magnetostrictive FeGaB and piezoelectric AIN ME heterostructures enables the demonstration of an $\mathrm{E}$ - and $\mathrm{H}$-field-tunable bandpass filter. Fig. 29(a) shows the schematic of the layered structure of the tunable MEMS filter, in which a gap of $2 \mu \mathrm{m}$ is designed between two ring-shaped FBAR resonators with FeGaB/AIN multilayer. Due to the strong ME coupling within the ME heterostructures, the acoustic waves can be strongly coupled to the 
electromagnetic waves. The measured $S$ parameters of the filter under zero bias field is presented in Fig. 29(b), showing a return loss of $-11.15 \mathrm{~dB}$ and insertion loss of $3.57 \mathrm{~dB}$ at the operation frequency of $93.165 \mathrm{MHz}$, with a Q-factor of 252. Because of the $\Delta \mathrm{E}$ effect, which changes the Young's modulus of a magnetostrictive material under a magnetic field, the central frequency of the ME filter will change when a magnetic field is applied. In Fig. 29(c), the measured central frequency as a function of applied DC magnetic field is presented, and a frequency tunability of $50 \mathrm{~Hz} / \mu \mathrm{T}$ is achieved. By applying a DC bias voltage, the E-field frequency tunability is extracted to be $2.3 \mathrm{kHz} / \mathrm{V}$, as shown in Fig. 29(d). This tunable RF bandpass filter, based on MEMS technology, is compact and compatible with CMOS technology.

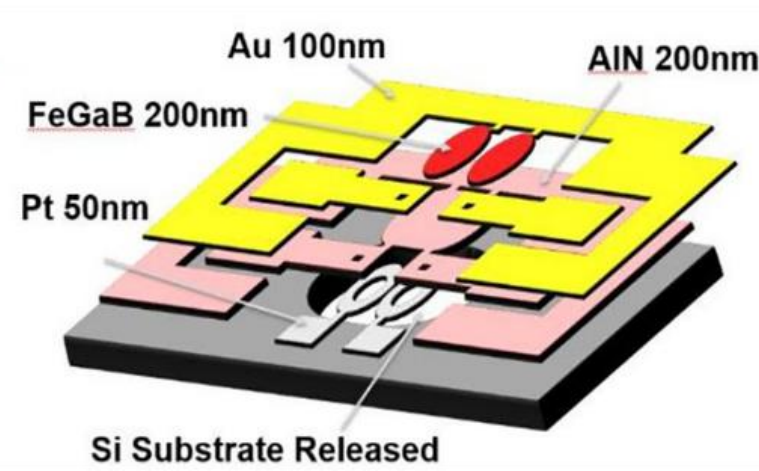

(a)

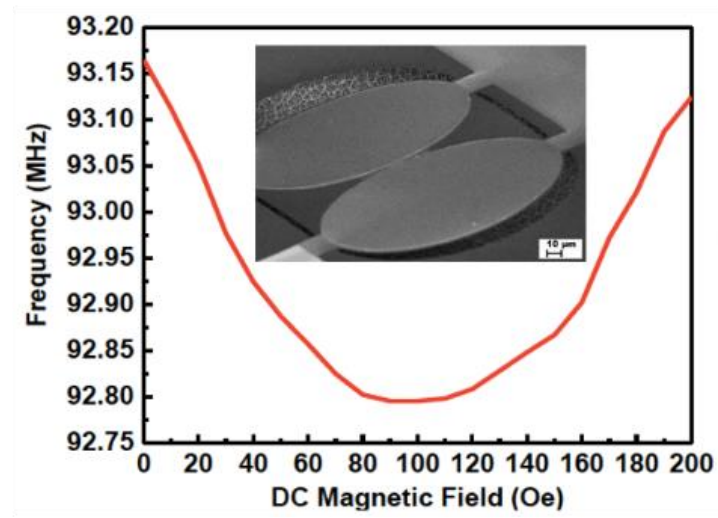

(c)

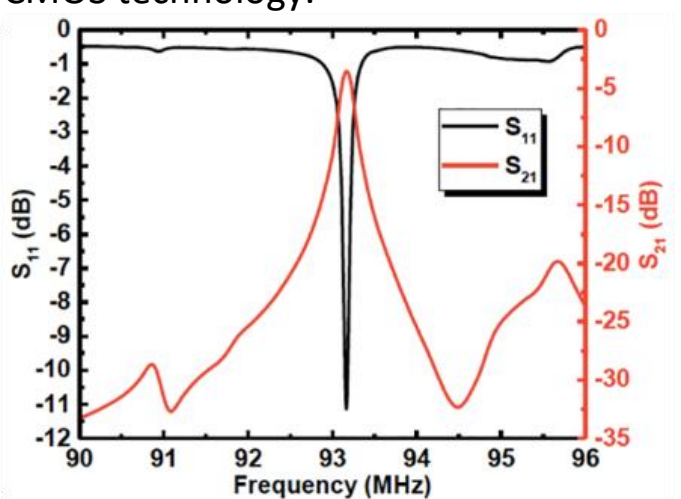

(b)

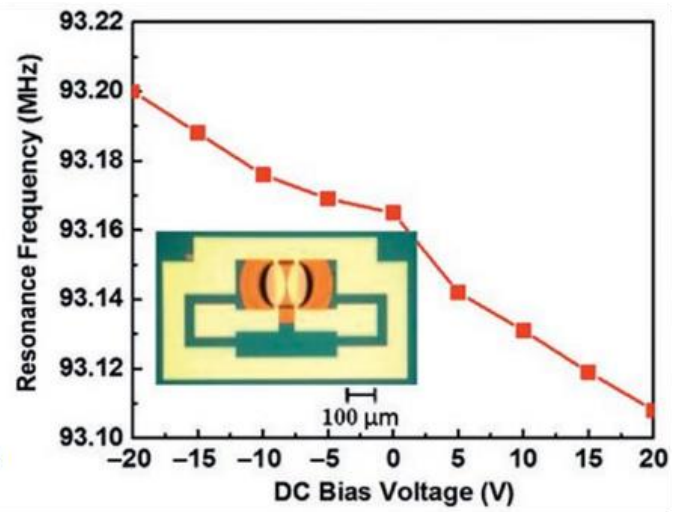

(d)

Fig. 29. (a) Schematic of the ME filter with two coupled ring-shaped FBAR resonators. (b) Measured S parameters of the ME filter under zero bias field. (c) Measured resonant frequency as a function of applied DC magnetic field. Inset shows the SEM image of the released ring-shaped resonators. (d) Measured resonant frequency versus DC bias voltage across thickness of the AIN film. Inset shows the top-down optical image of the ME filter. Reprinted with permission from $\mathrm{H}$. Lin et al. IEEE MTT-S International Microwave Symposium, 2016 [40]. Copyright [2016], IEEE.

\subsubsection{Isolators and Gyrators}

Non-reciprocal isolators, which allows the microwave power transmission in one direction only, have unique applications for signal processing in electronic systems. In the microwave frequency range, ferrite-based waveguides are the most common way to realize the non-reciprocal isolator 
[469]. However, they are not suitable for applications below $\mathrm{MHz}$ because of their heavy and large construction. In the low frequency range, active circuits with transistors and operational amplifiers are more appropriate for the realization of non-reciprocal isolators [470]. For scenarios requiring zero power consumption, such active circuits are avoided. Tu et al. recently demonstrated a passive isolator based on ME laminate composites [471]. The gyrator based on ME interactions was first proposed by B. Tellegen [472] and demonstrated by S. Dong [473] et al. by utilizing magnetostrictive/piezoelectric laminate composites. The size of isolators constructed by using such ME gyrators is much smaller than that of ferrite-based isolators. The schematic of the two-port ME gyrator is shown in Fig. 30(a), in which the PZT slab is sandwiched between two stacks of Metglas layers. The ME laminate is wrapped by a 30-turn enameled copper coil, which serves as port 1 . The electrodes on the bottom and top of the PZT slab are defined as port 2. Fig. 30 (b) details the equivalent circuit model of the proposed gyrator and isolator, where $R_{m}, L_{m}, C_{m}$, are the motional resistance, inductance and capacitance of the mechanical resonator; $R_{p}$ and $C_{p}$ denote the resistance and capacitance of the PZT layer; $\eta$ represents the gyration ratio; $R_{c}$ and $L_{c}$ are the resistance and inductance of the coil; $Z_{L}$ is the load impedance; $L_{1}, L_{2}, C_{1}, C_{2}$ are the inductance and capacitance of the impedance matching networks. The measured and calculated $S$ parameters of the two-port ME isolator are presented in Fig. 30(c) and (d). The forward and insertion loss and reverse isolation at the operation frequency of $125 \mathrm{kHz}$ are $4.7 \mathrm{~dB}$ and $19 \mathrm{~dB}$, respectively. This indicates that signal power is transmitted in the forward direction and considerably diminished in the reverse direction. A good impedance matching at port 1 and 2 can be seen from $S_{11}$ and $S_{22}$ results as shown in Fig. 30(d). The calculated magnitudes of $S$ parameters from the equivalent circuit in Fig. 30(b) are also plotted in Fig. 30(c) and (d). The good agreement between the measured and calculated data verifies the accuracy and validity of the proposed equivalent circuit model. 


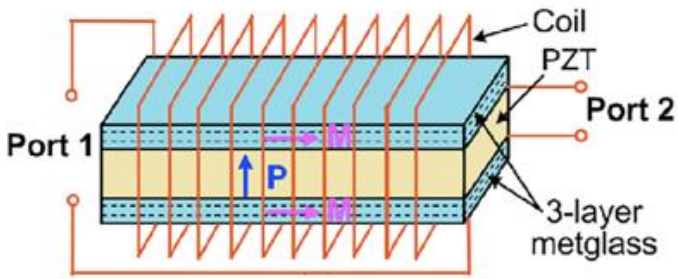

(a)

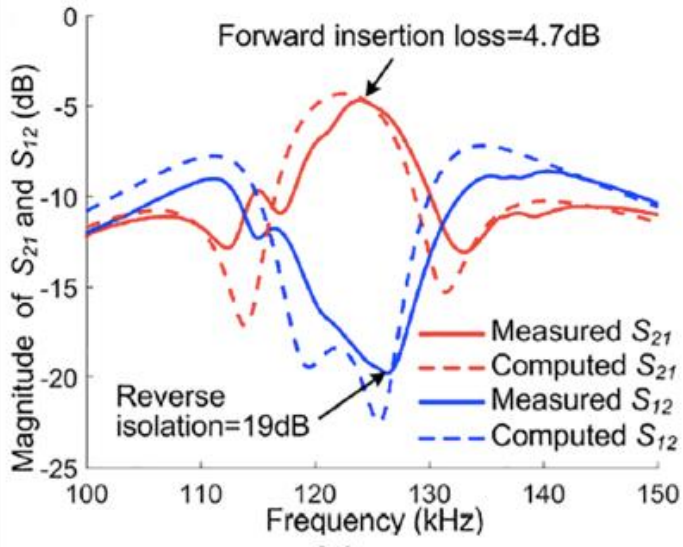

(c)

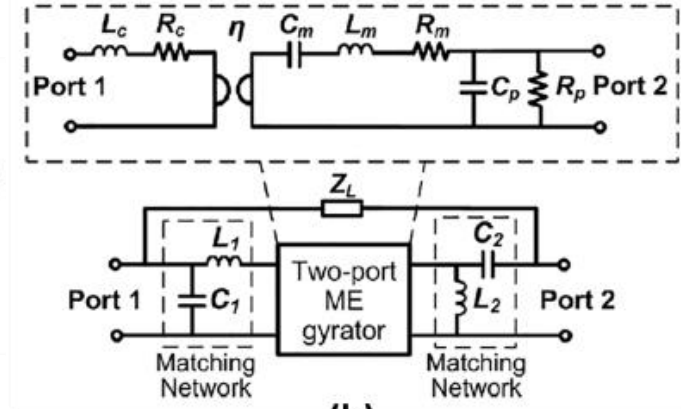

(b)

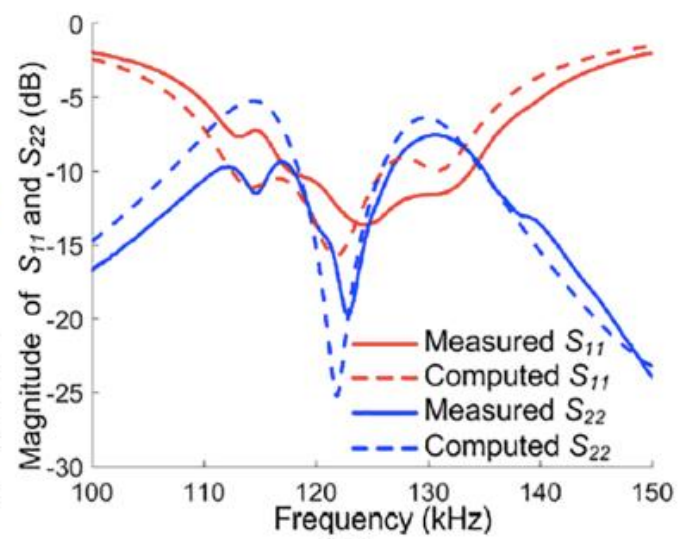

(d)

Fig. 30. (a) Schematic of the two-port ME gyrator. (b) Equivalent circuit model of the proposed gyrator and isolator. (c) Measured and calculated $S_{12}$ and $S_{21}$ of the ME isolator. (d) Measured and calculated $S_{11}$ and $S_{22}$ of the ME isolator. Reproduced with permission from $C$. Tu et al. Applied Physics Letters, 262904, 2018 [471]. Copyright [2018], AIP Publishing.

\section{Conclusions}

During the last decade, a variety of ME devices based on the strain-mediated piezoelectric/magnetostrictive heterostructures have been built and their performance has been enhanced significantly thanks to the strong ME coupling at the mechanical resonance. In turn, novel mechanisms to induce converse ME effects (such as electric surface charging or magnetoionics) are emerging and can pave the way towards new energy-efficient device functionalities, particularly in the domain of information technologies. In this review paper, we mainly devoted our efforts to the description and performance of different types of ME mechanisms, materials and devices that have attained a great deal of interest over recent years. Although lots of studies have been done on these systems, many opportunities and challenges still need to be addressed in the coming years. From the scientific point of view, disentangling of co-existing ME mechanisms for better design of materials, as well as understanding, modelling and characterizing of different devices incorporating piezoelectric/magnetostrictive heterostructures are of great importance for the multiferroic community. From the materials point of view, new ferroelectric materials with larger piezoelectric coefficients and new magnetostrictive materials with higher piezomagnetic coefficients are always desired. Concerning magneto-ionics, new 
engineering procedures to approach ns switching rates and improve cyclability are highly needed. This could be accomplished by precise control of the microstructure and ME coupling at $<10 \mathrm{~nm}$ length scales as well as exploring protocols to use new ion species, such as hydrogen. From the perspective of engineering applications, integration and scale-up with existing silicon processing flows and electronics are in particularly valuable. Low-temperature process methods are desired for fabricating RF multiferroic devices with low loss as well. In addition, flexible ME devices that can be compatible with wearable electronics are also significant for developing functional device applications. Through better understanding of the mechanisms of ME materials and devices, the challenges for multifunctional ME devices may be overcome and the promising future for applications realized. These research activities will likely facilitate developments within the multiferroic research community that will lead to large technological impacts on our daily lives.

Funding: P. Hayes, V. Schell, E. Quandt and J. McCord were funded by the German Research Foundation (Deutsche Forschungsgemeinschaft, DFG) through the Collaborative Research Centre CRC 1261 "Magnetoelectric Sensors: From Composite Materials to Biomagnetic Diagnostics". N. Sun acknowledges the financial support from the NSF TANMS ERC Award 1160504, W.M. Keck Foundation. Marisa Medarde acknowledges support by the Swiss National Science Foundation (Grants No. 200021-141334 and 206021_139082), the Swiss National Center of Competence in Research MARVEL (Computational Design and Discovery of Novel Materials, Grant No. 1NF40_182892) and the European Community's 7th Framework Program (Grant No. 290605; COFUND: PSI-FELLOW). P. Martins and S. Lanceros-Mendez thank the FCT- Fundação para a Ciência e Tecnologia- for financial support in the framework of the Strategic Funding UID/FIS/04650/2020 and under projects PTDC/BTM-MAT/28237/2017 and PTDC/EMDEMD/28159/2017. P. Martins thanks FCT- Fundação para a Ciência e Tecnologia for the contract under the Stimulus of Scientific Employment, Individual Support - 2017 Call (CEECIND/03975/2017). P. Martins and S. Lanceros-Mendez also thank funding by the Spanish State Research Agency (AEI) and the European Regional Development Fund (ERFD) through the project PID2019-106099RB-C43 / AEI / 10.13039/501100011033 and from the Basque Government Industry and Education Department under the ELKARTEK, HAZITEK and PIBA (PIBA2018-06) programs, respectively. S. van Dijken thanks the Academy of Finland for financial support under project no. 316857. J. Sort thanks financial support by the European Research Council (SPIN-PORICS 2014-Consolidator Grant, Agreement № 648454), the Spanish Government (MAT2017-86357-C3-1-R), the Generalitat de Catalunya (2017-SGR-292 and 2018-LLAV-00032) and the European Regional Development Fund (MAT2017-86357-C3-1-R and 2018-LLAV-00032).

Conflicts of Interest: The authors declare no conflict of interest. 


\section{References}

[1] P. Curie, "Sur la symétrie dans les phénomènes physiques, symétrie d'un champ électrique et d'un champ magnétique," J. Physique, vol. 3, no. 1, pp. 393-415, 1894

[2] D.N. Astrov, "The magnetoelectric effect in antiferromagnetics," Sov. Phys. J. Exp. Theor. Phys., vol. 11, no. 3, pp. 708-709, 1960.

[3] H. Schmid, "Multi-ferroic magnetoelectrics," Ferroelectrics, vol. 162, no. 1, pp. 317-338, 1994.

[4] Y. Wang, J. Hu, Y. Lin and C.-W. Nan," Multiferroic magnetoelectric composite nanostructures," NPG Asia Mater., vol. 2, pp. 61-68, 2010.

[5] S. Dong et al., "Multiferroic materials and magnetoelectric physics: symmetry, entanglement, excitation, and topology," Adv. Phys., vol. 64, no. 5-6, pp. 519-629, 2015.

[6] P. Zubko, G. Catalan, and A. K. Tagantsev, "Flexoelectric effect in sòlids," Annu. Rev. Mater. Res., vol. 43, no.1, pp. 387-421, 2013.

[7] C. Song, B. Cui, F. Li, X. Zhou, and F. Pan, "Recent progress in voltage control of magnetism: Materials, mechanisms, and performance," Prog. Mater. Sci., vol. 87, pp. 33-82, 2017.

[8] A. Molinari, H. Hahn, and R. Kruk, "Voltage-Control of Magnetism in All-Solid-State and Solid/Liquid Magnetoelectric Composites," Adv. Mater., vol. 31, no.28, Art. No. 1806662, 2019.

[9] C. Navarro-Senent, A. Quintana, E. Menéndez, E. Pellicer, J. Sort, "Electrolyte-gated magnetoelectric actuation: Phenomenology, materials, mechanisms, and prospective Applications," APL Mater., vol.7, no. 3, Art. No. 030701, 2019.

[10] A. Nicolenco A, M. de h-Óra , C. Yun , J. MacManus-Driscoll, J. Sort, "Perspective: Straingradient effects in nanoscale-engineered magnetoelectric materials," APL Materials, 2021 (in press).

[11] G. Srinivasan, "Magnetoelectric composites," Annu. Rev. Mater. Res., vol. 40, pp. 153-178, 2010.

[12] N. A. Spaldin and M. Fiebig, "The renaissance of magnetoelectric multiferroics," Science, vol. 309, no. 5733, pp. 391-392, 2005.

[13] M. Fiebig, "Revival of the magnetoelectric effect," J. Phys. D: Appl. Phys., vol. 38, no. 8, pp. R123-R152, 2005.

[14] W. Eerenstein, N. Mathur, and J. F. Scott, "Multiferroic and magnetoelectric materials," Nature, vol. 442, no. 7104, pp. 759-765, 2006.

[15] R. Ramesh and N. A. Spaldin, "Multiferroics: progress and prospects in thin films," Nat. Mater., vol. 6, no. 1, pp. 21-29, 2007.

[16] C.-W. Nan, M. Bichurin, S. Dong, D. Viehland, and G. Srinivasan, "Multiferroic magnetoelectric composites: Historical perspective, status, and future directions," J. Appl. Phys., vol. 103, no. 3, Art. No. 031101, 2008. 
[17] L. W. Martin, Y.-H. Chu, and R. Ramesh, "Advances in the growth and characterization of magnetic, ferroelectric, and multiferroic oxide thin films," Mater. Sci. Eng. R Rep., vol. 68, no. 46, pp. 89-133, 2010.

[18] J. Ma, J. Hu, Z. Li, and C. W. Nan, "Recent progress in multiferroic magnetoelectric composites: from bulk to thin films," Adv. Mater., vol. 23, no. 9, pp. 1062-1087, 2011.

[19] N. X. Sun and G. Srinivasan, "Voltage control of magnetism in multiferroic heterostructures and devices," Spin, vol. 2, no. 03, Art. No. 1240004, 2012.

[20] H. Palneedi, V. Annapureddy, S. Priya, and J. Ryu, "Status and perspectives of multiferroic magnetoelectric composite materials and applications," Actuators, 2016, vol. 5, no. 1, Art. No. 9, 2016.

[21] E. Gradauskaite, P. Meisenheimer, M. Müller, J. Heron, M. Trassin, "Multiferroic heterostructures for spintronics," Phys. Sci. Rev., Art. No. 20190072, 2020.

[22] X. Liang et al., "A Review of Thin-Film Magnetoelastic Materials for Magnetoelectric Applications," Sensors, vol. 20, no. 5, Art. No. 1532, 2020.

[23] X. Liang et al., "Multiferroic Composites," Elsevier, 2020.

[24] X. Liang, H. Chen, N. Sun, H. Lin, and N. X. Sun, "Novel Acoustically Actuated Magnetoelectric Antennas," IEEE International Symposium on Antennas and Propagation \& USNC/URSI National Radio Science Meeting, pp. 2189-2190, 2018.

[25] T. Nan et al., "Acoustically actuated ultra-compact NEMS magnetoelectric antennas," Nat. Commun., vol. 8, no. 1, Art. No. 296, 2017.

[26] C. Dong et al., "A Portable Very Low Frequency (VLF) Communication System Based on Acoustically Actuated Magnetoelectric Antennas," IEEE Antennas Wirel. Propag. Lett., vol. 19, no. 3, pp. 398-402, 2020.

[27] H. Chen et al., "Ultra-compact mechanical antennas," Appl. Phys. Lett., vol. 117, no. 17, Art. No. 170501, 2020.

[28] J. A. Bickford, A. E. Duwel, M. S. Weinberg, R. S. McNabb, D. K. Freeman, and P. A. Ward, "Performance of electrically small conventional and mechanical antennas," IEEE Trans. Antennas Propag., vol. 67, no. 4, pp. 2209-2223, 2019.

[29] M. A. Kemp et al., "A high Q piezoelectric resonator as a portable VLF transmitter," Nat. Commun., vol. 10, no. 1, Art. No. 1715, 2019.

[30] M. Li et al., "Highly sensitive DC magnetic field sensor based on nonlinear ME effect," IEEE Sensors Lett., vol. 1, no. 6, Art. No. 2501104, 2017.

[31] C. Tu et al., "Mechanical-Resonance-Enhanced Thin-Film Magnetoelectric Heterostructures for Magnetometers, Mechanical Antennas, Tunable RF Inductors, and Filters," Materials, vol. 12, no. 14, p. 2259, 2019.

[32] T. Nan, Y. Hui, M. Rinaldi, and N. X. Sun, "Self-biased $215 \mathrm{MHz}$ magnetoelectric NEMS resonator for ultra-sensitive DC magnetic field detection," Sci. Rep., vol. 3, Art. No. 1985, 2013. 
[33] Z. Chu et al., "A low-power and high-sensitivity magnetic field sensor based on converse magnetoelectric effect," Appl. Phys. Lett., vol. 115, no. 16, Art. No. 162901, 2019.

[34] Y. Wang, J. Li, and D. Viehland, "Magnetoelectrics for magnetic sensor applications: status, challenges and perspectives," Mater. Today, vol. 17, no. 6, pp. 269-275, 2014.

[35] Y. Gao et al., "Power-efficient voltage tunable RF integrated magnetoelectric inductors with FeGaB/Al $\mathrm{O}_{3}$ multilayer films," in 2014 IEEE MTT-S International Microwave Symposium (IMS2014), 2014, pp. 1-4.

[36] H. Chen et al., "Integrated Tunable Magnetoelectric RF Inductors," IEEE Trans Microw Theory Tech., vol. 68, no. 3, pp. 951-963, 2020.

[37] S. S. Bedair, J. S. Pulskamp, C. D. Meyer, M. Mirabelli, R. G. Polcawich, and B. Morgan, "Highperformance micromachined inductors tunable by lead zirconate titanate actuators," IEEE Electron Dev. Lett., vol. 33, no. 10, pp. 1483-1485, 2012.

[38] H. Lin et al., "Voltage tunable magnetoelectric inductors with improved operational frequency and quality factor for power electronics," IEEE Trans. Magn., vol. 51, no. 1, Art. No. 4002705, 2014.

[39] H. Lin et al., "Integrated non-reciprocal dual H-and E-Field tunable bandpass filter with ultrawideband isolation," 2015 IEEE MTT-S International Microwave Symposium, 2015, pp. 1-4.

[40] H. Lin et al., "Tunable RF band-pass filters based on NEMS magnetoelectric resonators," IEEE MTT-S International Microwave Symposium (IMS), 2016, pp. 1-4.

[41] A. Tatarenko, V. Gheevarughese, and G. Srinivasan, "Magnetoelectric microwave bandpass filter," Electron. Lett., vol. 42, no. 9, pp. 540-541, 2006.

[42] A. Ustinov, G. Srinivasan, and B. Kalinikos, "Ferrite-ferroelectric hybrid wave phase shifters," Appl. Phys. Lett., vol. 90, no. 3, Art. No. 031913, 2007.

[43] M. Gajek et al.,"Tunnel junctions with multiferroic barriers," Nat. Mater., vol. 6, pp. 296302, 2007.

[44] C. Binek, B. Doudin, "Magnetoelectronics with magnetoelectrics," J. Phys.: Condens. Matter, vol. 17, no. 2, pp. L39-L44, 2005.

[45] H. K. D. Kim, L. Schelhas, S. Keller, J.L. Hockel, S. H. Tolbert and G. P. Carman, "Magnetoelectric Control of Superparamagnetism," Nano Lett., vol. 13, no.3, pp. 884-888, 2013.

[46] W. Chen, M. Sigrist, “Dissipationless Multiferroic Magnonics," Phys. Rev. Lett., vol.114, no.15, Art. No. 157203, 2015.

[47] R. Guduru et al., "Magnetoelectric 'spin' on stimulating the brain," Nanomedicine, vol. 10, no. 13, pp. 2051, 2015.

[48] M. Nair et al., "Externally controlled on-demand release of anti-HIV drug using magnetoelectric nanoparticles as carriers," Nat. Commun., vol. 4, Art. No. 1707, 2013. 
[49] X.-Z. Chen et al., "Hybrid Magnetoelectric Nanowires for Nanorobotic Applications: Fabrication, Magnetoelectric Coupling, and Magnetically Assisted In Vitro Targeted Drug Delivery," Adv. Mater., vol. 29, no. 8, Art. No. 1605458, 2017.

[50] C. Fermon and M. Pannetier-Lecoeur, "Noise in GMR and TMR Sensors," Giant Magnetoresistance (GMR) Sensors, vol. 6, Berlin, Heidelberg: Springer Berlin Heidelberg, pp. 4770, 2013.

[51] N. A. Stutzke et al., "Low-frequency noise measurements on commercial magnetoresistive magnetic field sensors," J. Appl. Phys., vol. 97, no. 10, p. 10Q107, 2005.

[52] Y. Guo et al., "Reduction of magnetic $1 / \mathrm{f}$ noise in miniature anisotropic magnetoresistive sensors," Appl. Phys. Lett., vol. 106, no. 21, p. 212402, 2015.

[53] Y. J. Wang et al., "A review on equivalent magnetic noise of magnetoelectric laminate sensors," Philos. Trans. A Math. Phys. Eng. Sci., vol. 372, no. 2009, pp. 20120455, 2014.

[54] E. Yarar et al., "Inverse bilayer magnetoelectric thin film sensor," Appl. Phys. Lett., vol. 109, no. 2, Art. No. 022901, 2016.

[55] T. Wolf et al., "Subpicotesla Diamond Magnetometry," Phys. Rev. X, vol. 5, no. 4, p. 041001, 2015.

[56] J. R. Vig and F. L. Walls, "A review of sensor sensitivity and stability," in Proceedings of the 2000 IEEE/EIA International Frequency Control Symposium and Exhibition (Cat. No. 00CH37052), 2000, pp. 30-33: IEEE.

[57] J.F. Scott, "Room-temperature multiferroic magnetoelectrics," Npg Asia Mater., vol. 5, Art. No. e72, 2013.

[58] Y. Tokura, S. Seki, N. Nagaosa, "Multiferroics of spin origin," Rep. Prog. Phys., vol. 77, no. 7, Art. No. 076501, 2014.

[59] T. Kimura, "Magnetoelectric Hexaferrites," Annu. Rev. Conden. Matter Phys., vol. 3, no. 1, pp. 93-110, 2012.

[60] M. Fiebig, T. Lottermoser, D. Meier, M. Trassin, "The evolution of multiferroics," Nat. Rev. Mater., vol 1, no. 8, p. 16046, 2016.

[61] N. A. Spaldin, "Multiferroics beyond electric-field control of magnetism," Proc. R. Soc. A., vol. 476, no. 2233, Art. No. 20190542,2020.

[62] N. Spaldin and R. Ramesh, "Advances in magnetoelectric multiferroics," Nat. Mater., vol. 18, no. 3, pp. 203-212, 2019.

[63] C.W. Cheong, M. Fiebig, W.D. Wu, L. Chapon, V. Kiryukhin, "Seeing is believing: visualization of antiferromagnetic domains," Npj Quantum Mater., vol. 5, no. 1, Art. No. 3, 2020.

[64] F. Matsukura, Y. Tokura, H. Ohno, "Control of magnetism by electric fields," Nat. Nanotechnol., vol. 10, no. 3, pp. 209-220, 2015.

[65] N. A. Hill, "Why are there so few magnetic ferroelectrics?" J. Phys. Chem. B, vol. 104, no. 29, pp. 6694-6709, 2000. 
[66] G. Catalan, J. F. Scott, "Physics and Applications of Bismuth Ferrite," Adv. Mater., vol 21, no. 24, pp. 2463-2485, 2009.

[67] D. Lebeugle, D. Colson, A. Forget, M. Viret, "Very large spontaneous electric polarization in $\mathrm{BiFeO}_{3}$ single crystals at room temperature and its evolution under cycling fields," Appl. Phys. Lett., vol. 91, no. 2, Art. No. 022907, 2007.

[68] D. Lebeugle, D. Colson, A. Forget, M. Viret, , P. Bonville, J. F. Marucco, "Room-temperature coexistence of large electric polarization and magnetic order in BiFeO3 single crystals," Phys. Rev. B, vol. 76, no. 2, Art. No. 024116, 2007.

[69] I. Sosnowska, T. Peterlinneumaier, E. Steichele, "Spiral Magnetic-Ordering in Bismuth Ferrite", J. Phys. C. Solid. State, vol. 15, no. 23, pp. 4835-4846, 1982.

[70] D. Lebeugle, D. Colson, A. Forget, M. Viret, A.M. Bataille,A. Gukasov, "Electric-field-induced spin flop in $\mathrm{BiFeO}_{3}$ single crystals at room temperature," Phys. Rev. Lett., vol. 100, no. 22, Art. No. $227602,2008$.

[71] Y. F. Popov,, A. K. Zvezdin, G. P. Vorobev, A. M. Kadomtseva, V. A. Murashev, D. N. Rakov,. "Linear Magnetoelectric Effect and Phase-Transitions in Bismuth Ferrite, BiFeO $\mathrm{B}_{3}$, Jetp. Lett., vol. 57, no. 1, pp. 69-73, 1993.

[72] M. Matsuda et al., "Magnetic field induced antiferromagnetic cone structure in multiferroic $\mathrm{BiFeO}_{3}$," Phys. Rev. Mater., vol 4, no. 3, pp. 034412, 2020.

[73] D. Lebeugle, A. Mougin, M. Viret, D. Colson, L. Ranno, "Electric Field Switching of the Magnetic Anisotropy of a Ferromagnetic Layer Exchange Coupled to the Multiferroic Compound $\mathrm{BiFeO}_{3}$," Phys. Rev. Lett., vol. 103, no. 25, pp. 257601, 2009.

[74] J. T. Heron et al.,, "Deterministic switching of ferromagnetism at room temperature using an electric field," Nature, vol. 516, p. 370, 2014.

[75] S. Manipatruni et al., "Scalable energy-efficient magnetoelectric spin-orbit logic," Nature, vol. 565, p. 35, 2019.

[76] A. Moure, "Review and Perspectives of Aurivillius Structures as a Lead-Free Piezoelectric System," Appl. Sci.-Basel, vol. 8, no. 1, p. 62, 2018.

[77] C. A. P. Dearaujo, J.D. Cuchiaro, L.D. Mcmillan,, M.C. Scott, J. F. Scott, "Fatigue-Free Ferroelectric Capacitors with Platinum-Electrodes," Nature, vol. 374, no. 6523, pp. 627-629, 1995.

[78] B. H. Park, B. S. Kang, S. D. Bu, T. W. Noh, J. Lee, W. Jo, "Lanthanum-substituted bismuth titanate for use in non-volatile memories," Nature, vol. 401, no. 6754, pp. 682-684, 1999.

[79] I. G. Ismailzade, V. I. Nesterenko, F. A. Mirishli, P. G. Rustamov, “ X-Ray and Electrical Studies of System $\mathrm{Bi}_{4} \mathrm{Ti}_{3} \mathrm{O}_{12}-\mathrm{BiFeO}_{3}$," Sov. Phys. Crystallogr., vol. 12, no. 3, pp. 400-404, 1967.

[80] A. Y. Birenbaum, C. Ederer, "Potentially multiferroic Aurivillius phase Bi5FeTi3015: Cation site preference, electric polarization, and magnetic coupling from first principles," Phys. Rev. $B$, vol. 90, no. 21, Art. No. 214109, 2014.

[81] X. Y. Mao, W. Wang, X.B. Chen, Y. L. Lu, "Multiferroic properties of layer-structured $\mathrm{Bi}_{5} \mathrm{Fe}_{0.5} \mathrm{Co}_{0.5} \mathrm{Ti}_{3} \mathrm{O}_{15}$ ceramics," Appl. Phys. Lett., vol. 95, no. 8, Art. No. 082901, 2009. 
[82] X. Y. Mao, H. Sun, W. Wang, Y. L. Lu, X. B. Chen, "Effects of Co-substitutes on multiferroic properties of $\mathrm{Bi}_{5} \mathrm{FeTi}_{3} \mathrm{O}_{15}$ ceramics," Solid State Commun., vol. 152, no. 6, pp. 483-487, 2012.

[83] W. Bai et al., "Electrical, magnetic, and optical properties in multiferroic $\mathrm{Bi}_{5} \mathrm{Ti}_{3} \mathrm{FeO}_{15}$ thin films prepared by a chemical solution deposition route," J. Appl. Phys., vol. 109, no. 6, Art. No. 064901, 2011.

[84] T. Chen et al., "Intrinsic multiferroics in an individual single-crystalline $\mathrm{Bi}_{5} \mathrm{Fe}_{0.9} \mathrm{Co}_{0.1} \mathrm{Ti}_{3} \mathrm{O}_{15}$ nanoplate," Nanoscale, vol. 9, no. 40, pp. 15291-15297, 2017.

[85] S. J. Sun et al., "Synthesis of Ni-substituted $\mathrm{Bi}_{7} \mathrm{Fe}_{3} \mathrm{Ti}_{3} \mathrm{O}_{21}$ ceramics and their superior room temperature multiferroic properties," RSC Adv., vol.3, no.40, pp. 18567-18572, 2013.

[86] L. Keeney et al., "Room temperature ferroelectric and magnetic investigations and detailed phase analysis of Aurivillius phase $\mathrm{Bi}_{5} \mathrm{Ti}_{3} \mathrm{Fe}_{0.7} \mathrm{Co}_{0.3} \mathrm{O}_{15}$ thin films," J. Appl. Phys., vol. 112, no. 5, Art. No. 052010, 2012.

[87] M. M. Kumar, A. Srinivas, S.V. Suryanarayana, G. S. Kumar, T. Bhimasankaram, "An experimental setup for dynamic measurement of magnetoelectric effect," Bull. Mater. Sci., vol. 21, no.3, pp. 251-255, 1998.

[88] S. V. Suryanarayana, "Magnetoelectric Interaction Phenomena in Materials," Bull. Mater. Sci., vol. 17, no. 7, pp. 1259-1270, 1994.

[89] L. Fuentes, M. Garcia, J. Matutes-Aquino, D. Rios-Jara, "Magnetoelectricity via crystallography," J. Alloy Compd., vol. 369, no. 1-2, pp. 10-13, 2004.

[90] J. L. Wang et al., "Low magnetic field response single-phase multiferroics under high temperature," Mater. Horiz., vol. 2, no.2, pp. 232-236, 2015.

[91] R. C. Pullar, "Hexagonal ferrites: A review of the synthesis, properties and applications of hexaferrite ceramics," Prog. Mater. Sci., vol. 57, no. 7, pp. 1191-1334, 2012.

[92] T. Kimura., G. Lawes, A. P. Ramirez, "Electric polarization rotation in a hexaferrite with longwavelength magnetic structures," Phys. Rev. Lett., vol. 94, no. 13, Art. No. 137201, 2005.

[93] Y. Kitagawa, Y. Hiraoka, T. Honda, T. Ishikura, H. Nakamura, T. Kimura, "Low-field magnetoelectric effect at room temperature," Nat. Mater., vol. 9, no. 10, pp. 797-802, 2010.

[94] P. Novak, K. Knizek, J. Rusz, "Magnetism in the magnetoelectric hexaferrite system ( $\mathrm{Ba}_{1-}$ $\left.{ }_{x} \mathrm{Sr}_{\mathrm{x}}\right)_{2} \mathrm{Zn}_{2} \mathrm{Fe}_{12} \mathrm{O}_{22}$," Phys. Rev. B, vol. 76, no. 2, Art. No. 024432, 2007.

[95] H. Ueda, Y. Tanaka, Y. Wakabayashi, T. Kimura, "Insights into magnetoelectric coupling mechanism of the room-temperature multiferroic $\mathrm{Sr}_{3} \mathrm{Co}_{2} \mathrm{Fe}_{24} \mathrm{O}_{41}$ from domain observation," Phys. Rev. B, vol. 100, no. 9, Art. No. 094444, 2019.

[96] M. Morin et al., "Incommensurate magnetic structure, $\mathrm{Fe} / \mathrm{Cu}$ chemical disorder, and magnetic interactions in the high-temperature multiferroic $\mathrm{YBaCuFeO}_{5}$," Phys. Rev. $B$, vol. 91, no. 6, Art. No. 064408, 2015.

[97] Y. C. Lai, G. J. Shu, W. T. Chen, C. H. Du, F. C. Chou, "Self-adjusted flux for the traveling solvent

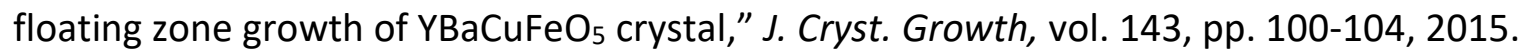


[98] M. K. Srivastava et al., "The effect of orbital-lattice coupling on the electrical resistivity of $\mathrm{YBaCuFeO}_{5}$ investigated by X-ray absorption," Sci. Rep., vol. 9, Art. No. 18586, 2019.

[99] Al. Klyndyuk and E. A. Chizhova, "Properties of $\mathrm{RBaCuFeO}_{5+\delta}(\mathrm{R}=\mathrm{Y}$, La, Pr, Nd, Sm-Lu)," Inorg. Mater., vol. 42, no. 5, pp. 550-561, 2006.

[100] T. Shang et al., "Design of magnetic spirals in layered perovskites: Extending the stability range far beyond room temperature," Sci. Adv., vol. 4, no. 10., Art. No. eaau6386, 2018.

[101] M. Morin et al., "Tuning magnetic spirals beyond room temperature with chemical disorder," Nat. Commun., vol. 7, Art. No. 13758, 2016.

[102] A. Scaramucci et al., "Multiferroic Magnetic Spirals Induced by Random Magnetic Exchanges", Phys. Rev. X, vol. 8, no. 1, p. 011005, 2018.

[103] A. Scaramucci, H. Shinaoka, M. V. Mostovoy, R. Lin,C. Mudry, M. Muller, "Spiral order from orientationally correlated random bonds in classical XY models", Phys. Rev. Res., vol. 2, no. 1, Art. No. 013273, 2020.

[104] B. Kundys, A. Maignan, C. Simon, "Multiferroicity with high-T-C in ceramics of the $\mathrm{YBaCuFeO}_{5}$ ordered perovskite", Appl. Phys. Lett., vol. 94, no. 7, Art. No. 072506, 2009.

[105] Y. Kawamura et al., "High-Temperature Multiferroic State of $\mathrm{RBaCuFeO}_{5}(\mathrm{R}=\mathrm{Y}$, $\mathrm{Lu}$, and Tm)", J. Phys. Soc. Jpn., vol. 79, no. 7, Art. No. 073705, 2010.

[106] V. Caignaert et al., "Crystal and Magnetic-Structure of $\mathrm{YBaCuFeO}_{5}$ ", J. Solid. State. Chem., vol. 114, no. 1, pp. 24-35, 1995.

[107] S. J. Luo, and K. F. Wang, "Giant dielectric permittivity and magneto-capacitance effect in YBaCuFeO 5 ", Scripta Mater., vol. 146, pp. 160-163, 2018.

[108] M. Soda, T. Ishikura, H. Nakamura, Y. Wakabayashi, and T. Kimura, "Magnetic Ordering in Relation to the Room-Temperature Magnetoelectric Effect of $\mathrm{Sr}_{3} \mathrm{Co}_{2} \mathrm{Fe}_{24} \mathrm{O}_{41}$ ", Phys. Rev. Lett., vol. 106, no. 8, Art. No. 087201, 2011.

[109] D. M. Evans, V. Garcia, D. Meier, M. Bibes, "Domains and domain walls in multiferroics," Phys. Sci. Rev., vol. 5, no. 9, pp. 227-240, 2020.

[110] J. Van Den Boomgaard, D. R. Terrel, R. A. J. Born, and H. F. J. I. Giller, "An in situ grown eutectic magnetoelectric composite material. Part I," J. Mater. Sci., vol. 9, pp. 1705-1709, 1974.

[111] A. M. J. G. van Run, D. R. Terrell, and J. H. Scholing, "An in situ grown eutectic magnetoelectric composite material. Part II," J. Mater. Sci., vol. 9, pp. 1710-1714, 1974.

[112] J. Van Den Boomgaard, A. M. J. G. van Run, and J. van Suchtelen, "Piezoelectricpiezomagnetic composites with magnetoelectric effect," Ferroelectrics, vol. 9, pp. 727-728, 1976.

[113] J. Van Den Boomgaard and R. A. J. Born, "A sintered magnetoelectric composite material $\mathrm{BaTiO}_{3}-\mathrm{Ni}(\mathrm{Co}, \mathrm{Mn}) \mathrm{Fe}_{2} \mathrm{O}_{4}$," J. Mater. Sci., vol. 13, pp. 1538-1548, 1978.

[114] G. Srinivasan, V. Laletsin, R. Hayes, N. Puddubnaya, E. T. Rasmussen, and D. J. Fekel, “Giant magnetoelectric effects in layered composites of nickel zinc ferrite and lead zirconate titanate", Solid State Commun., vol. 124, pp. 373-378, 2002. 
[115] J. Zhai, N. Cai, Z. Shi, Y. Lin, and C. W. Nan, "Magnetic-dielectric properties of $\mathrm{NiFe}_{2} \mathrm{O}_{4} / \mathrm{PZT}$ particulate composites," J. Phys. D: Appl. Phys., vol. 37, no. 6, pp 823-825, 2004.

[116] R. A. Islam, H. Kim, S. Priya, and H. Stephanou, "Piezoelectric transformer based ultrahigh sensitivity magnetic field sensor," Appl. Phys. Lett., vol. 89, Art. No. 152908, 2006.

[117] R. A. Islam, V. Bedekar, N. Poudyal, J. P. Liu, and S. Priya, "Magnetoelectric properties of core-shell particulate nanocomposites," J. Appl. Phys., vol. 104, Art. No. 104111, 2008.

[118] R. A. Islam and S. Priya, "Effect of piezoelectric grain size on magnetoelectric coefficient of $\mathrm{Pb}\left(\mathrm{Zr}_{0.52} \mathrm{Ti}_{0.48}\right) \mathrm{O}_{3}-\mathrm{Ni}_{0.8} \mathrm{ZnO}_{.2} \mathrm{Fe}_{2} \mathrm{O}_{4}$ particulate composites," J. Mater. Sci. 43, pp. 3560-3568, 2008.

[119] M. Zeng, J. G. Wan, Y. Wang, H. Yu, J.-M. Liu, X. P. Jiang, and C. W. Nan, "Resonance magnetoelectric effect in bulk composites of lead zirconate titanate and nickel ferrite", J. Appl. Phys., vol. 95, p. 8069, 2004.

[120] G. Srinivasan, R. C. P. DeVreugd, C. S. Flattery, V. M. Laletsin, and N. Paddubnaya, "Magnetoelectric interactions in hot-pressed nickel zinc ferrite and lead zirconante titanate composites," Appl. Phys. Lett., vol. 85, no. 13, pp. 2550-2552, 2004.

[121] D. Wu, W. Gong, H. Deng, and M. Li, "Magnetoelectric composite ceramics of nickel ferrite and lead zirconate titanate via in situ processing", J. Phys. D: Appl. Phys. vol. 40, no. 16, pp. 50025005, 2007.

[122] M. V. Ramanaa, N. R. Reddy, G. Sreenivasulu, K. V. Siva Kumar, B. S. Murty, and V. R. K. Murthy, "Enhanced mangnetoelectric voltage in multiferroic particulate $\mathrm{Ni}_{0.83} \mathrm{CO}_{0.15} \mathrm{Cu}_{0.02} \mathrm{Fe}_{1.9} \mathrm{O}_{4-\delta} / \mathrm{PbZr}_{0.52} \mathrm{Ti}_{0.48} \mathrm{O}_{3}$ composites - dielectric, piezoelectric and magnetic properties," Current Appl. Phys., vol. 9, no. 5, pp. 1134-1139, 2009.

[123] G. V. Duong, R. Groessinger, and R. S. Turtelli, "Magnetoelectric Properties of $\mathrm{CoFe}_{2} \mathrm{O}_{4}{ }^{-}$ $\mathrm{BaTiO}_{3}$ Core-Shell Structure Composite”, IEEE Trans. Magn., vol. 42, no. 10, pp. 3611-3613, 2006.

[124] D. K. Pradhan, S. Kumari and P. D. Rack, "Magnetoelectric Composites: Applications, Coupling Mechanisms, and Future Directions," Nanomaterials, vol. 10, Art. No. 2072, 2020.

[125] R. Liu, Y. Zhao, R. Huang, Y. Zhao, and H. Zhou, "Multiferroic ferrite/perovskite oxide core/shell nanostructures," J. Mater. Chem., vol. 20, pp. 10665-10670, 2010.

[126] L. P. Curecheriu et al., "Functional properties of $\mathrm{BaTiO}_{3}-\mathrm{Ni}_{0.5} \mathrm{Zn}_{0.5} \mathrm{Fe}_{2} \mathrm{O}_{4}$ magnetoelectric ceramics prepared from powders with core-shell structure," J. Appl. Phys., vol. 107, Art. No. 104106, 2010.

[127] G. Srinivasan, E. T. Rasmussen, J. Gallegos, R. Srinivasan, Y. I. Bokhan, and V. M. Laletin, "Magnetoelectric bilayer and multilayer structures of magnetostrictive and piezoelectric oxides", Phys. Rev. B, vol. 64, Art. No. 214408, 2001.

[128] G. Srinivasan, E. T. Rasmussen, and R. Hayes, "Magnetoelectric effects in ferrite-lead zirconate titanate layered composites: The influence of zinc substitution in ferrites", Phys. Rev. $B$, vol. 67, no. 1, Art. No. 014418, 2003. 
[129] G. Srinivasan, R. Hayes, and M. I. Bichurin, "Low frequency and microwave magnetoelectric effects in thick film heterostructures of lithium zinc ferrite and lead zirconate titanate," Sol. State Commun., vol. 128, no. 6-7, pp. 261-266, 2003.

[130] J. Ryu, S. Priya, A. V. Carazo, K. Uchino, and H. E. Kim, "Effect of the Magnetostrictive Layer on Magnetoelectric Properties in Lead Zirconate Titanate/Terfenol-D Laminate Composites," J. Am. Chem. Soc., vol. 84, no. 12, pp. 2905-2908, 2001.

[131] S. X. Dong, J. Y. Zhai, F. Bai, J. F. Li, and D. Viehland, "Push-pull mode magnetostrictive/piezoelectric laminate composite with an enhanced magnetoelectric voltage coefficient," Appl. Phys. Lett., vol. 87, Art. No. 062502, 2005.

[132] Y. J. Wang, D. Gray, D. Berry, J. Q. Gao, M. H. Li, J. F. Li, and D. Viehland, "An Extremely Low Equivalent Magnetic Noise Magnetoelectric Sensor," Adv. Mater., vol. 23, no. 35, pp. 4111-4114, 2011.

[133] M. Li, J. Gao, Y. Wang, D. Gray, J. Li, and D. Viehland, "Enhancement in magnetic field sensitivity and reduction in equivalent magnetic noise by magnetoelectric laminate stacks," J. Appl. Phys. Vol. 111, No. 10, Art. No. 104504, 2012.

[134] H. Zheng et al., "Multiferroic $\mathrm{BaTiO}_{3}-\mathrm{CoFe}_{2} \mathrm{O}_{4}$ Nanostructures," Science, vol. 303, no. 5658, pp. 661-663, 2004.

[135] A. Chen, Q. Su, H. Han, E. Enriquez, and Q. Jia, "Metal Oxide Nanocomposites: A Perspective from Strain, Defect, and Interface," Adv. Mater., vol. 31, no. 4, Art. No. 1803241, 2019.

[136] C. W. Nan, G. Liu, Y. H. Lin, and H. D. Chen, "Magnetic-Field-Induced Electric Polarization in Multiferroic Nanostructures," Phys. Rev. Lett., vol. 94, Art. No. 197203, 2005.

[137] J. X. Zhang et al., "Phase-field model for epitaxial ferroelectric and magnetic nanocomposite thin films," Appl. Phys. Lett., vol. 90, Art. No. 052909, 2007.

[138] N. A. Pertsev, H. Kohlstedt, and B. Dkhil, "Strong enhancement of the direct magnetoelectric effect in strained ferroelectric-ferromagnetic thin-film heterostructured," Phys. Rev. B, vol. 80, no. 5, Art. No. 054102, 2009.

[139] H. P. Wu, G. Z. Chai, T. Zhou, Z. Zhang, T. Kitamura, and H. M. Zhou, “Adjustable magnetoelectric effect of self-assembled vertical multiferroic nanocomposite films by the inplane misfit strain and ferromagnetic volume fraction," J. Appl. Phys., vol. 115, Art. No. 114105, 2014.

[140] Y. S. Oh, S. Crane, H. Zheng, Y. H. Chu, R. Ramesh, and K. H. Kim, "Quantitative determination of anisotropic magnetoelectric coupling in $\mathrm{BiFeO}_{3}-\mathrm{CoFe}_{2} \mathrm{O}_{4}$ nanostructures," Appl. Phys. Lett., vol. 97, Art. No. 052902, 2010.

[141] L. Yan, Z. P. Xing, Z. G. Wang, T. Wang, G. Y. Lei, J. F. Li, and D. Viehland, "Direct measurement of magnetoelectric exchange in self-assembled epitaxial $\mathrm{BiFeO}_{3}-\mathrm{CoFe}_{2} \mathrm{O}_{4}$ nanocomposite thin films," Appl. Phys. Lett., vol. 94, Art. No. 192902, 2009.

[142] T. Amrillah et al., " Flexible Multiferroic Bulk Heterojunction with Giant Magnetoelectric Coupling via van der Waals Epitaxy," ACS Nano, vol. 11, no. 6, pp. 6122-6130, 2017. 
[143] D. H. Kim, S. Ning, and C. A. Ross, "Self-assembled multiferroic perovskite-spinel nanocomposite thin films: epitaxial growth, templating and integration on silicon," J. Mater. Chem., vol. 7, pp. 9128-9148, 2019.

[144] H. Greve, E. Woltermann, H-J. Quenzer, B. Wagner, and E. Quandt, "Giant magnetoelectric coefficients in $\left(\mathrm{Fe}_{90} \mathrm{CO}_{10}\right)_{78} \mathrm{Si}_{12} \mathrm{~B}_{10}$-AIN thin film composites," Appl. Phys. Lett., vol. 96, Art. No. 182501, 2010.

[145] S. S. Nair, G. Pookat, V. Saravanan, and M. R. Anantharaman, "Lead free heterogeneous multilayers with giant magneto electric coupling for microelectronics/microelectromechanical systems Applications," J. Appl. Phys., vol. 114, Art. No. 064309, 2013.

[146] L. W. Martin et al., "Multiferroics and magnetoelectrics: thin films and nanostructures," J. Phys.: Condens. Matter, vol. 20, no. 43, Art. No. 434220, 2008.

[147] C. A. F. Vaz, J. Hoffman, C. H. Ahn, and R. Ramesh, "Magnetoelectric Coupling Effects in Multiferroic Complex Oxide Composite Structures", Adv. Mater., vol. 22, no. 36-27, pp. 29002918, 2010.

[148] G. Lawes and G. Srinivasan, " Introduction to magnetoelectric coupling and multiferroic films," J. Phys. D: Appl. Phys., vol. 44, no. 24, Art. No. 243001, 2011.

[149] N. Ortega, A. Kumar, J. F. Scott, and R. S. Katiyar, "Multifunctional magnetoelectric materials for device Applications," J. Phys.: Condens. Matter, vol.27, no. 50, Art. No. 504002, 2005.

[150] J-M. Hu, L-Q. Chen, and C-W. Nan, "Multiferroic Heterostructures Integrating Ferroelectric and Magnetic Materials," Adv. Mater., vol. 28, no. 1, pp. 15-39, 2016.

[151] D. Viehland et al., "Tutorial: Product properties in multiferroic nanocomposites," J. Appl. Phys., vol. 124, Art. No. 061101. 2018.

[152] Z. Chu, M. PourhosseiniAsl, and S. Dong, "Review of multi-layered magnetoelectric composite materials and devices Applications," J. Phys. D: Appl. Phys., vol. 51, no. 24, Art. No. 243001, 2018.

[153] C. M. Leung, J. Li, D. Viehland, and X Zhuang, "A review on applications of magnetoelectric composites: from heterostructural uncooled magnetic sensors, energy harvesters to highly efficient power converters," J. Phys. D: Appl. Phys., vol. 51, no. 26, Art. No. 263002, 2018.

[154] D. Viehland, M. Wuttig, J. McCord, and E. Quandt, "Magnetoelectric magnetic field sensors," MRS Bulletin, vol. 43, pp. 834-840, 2018.

[155] M. Bichurin, R. Petrov, V. Leontiev, G. Semenov, and O. Sokolov, "Magnetoelectric Current Sensors," Sensors, vol. 17, no. 6, Art. No. 1271, 2017.

[156] F. Narita and M. Fox, "A Review on Piezoelectric, Magnetostrictive, and Magnetoelectric Materials and Device Technologies for Energy Harvesting Applications," Adv. Eng. Mater., vol. 20, no. 5, Art. No. 1700743, 2018.

[157] C. A. F. Vaz, "Electric field control of magnetism in multiferroic heterostructured," J. Phys.: Condens. Matter, vol. 24, no. 33, Art. No. 333201, 2012. 
[158] T. Taniyama, "Electric-field control of magnetism via strain transfer across ferromagnetic/ferroelectric interfases," J. Phys.: Condens. Matter, vol. 27, no. 50, Art. No. 504001, 2015.

[159] F. Li, S. Zhang, Z. Xu, X. Wei, J. Luo, and T. R. Shrout, “Composition and phase dependence of the intrinsic and extrinsic piezoelectric activity of domain engineered (1-x) $\mathrm{Pb}\left(\mathrm{Mg}_{1 / 3} \mathrm{Nb}_{2 / 3}\right) \mathrm{O}_{3}-\mathrm{PPbTiO}_{3}$ crystals," J. Appl. Phys., vol. 108, no. 3, Art. No. 034106, 2010.

[160] C. Thiele, K. Dörr, O. Bilani, J. Rödel, and L. Schultz, "Influence of strain on the magnetization and magnetoelectric effect in La ${ }_{0.7} \mathrm{~A}_{0.3} \mathrm{MnO}_{3} / \mathrm{PMN}-\mathrm{PT}(001)(\mathrm{A}=\mathrm{Sr}, \mathrm{Ca})$," Phys. Rev. $B$, vol. 75, no. 5, Art. No. 054408, 2007.

[161] Z.G. Sheng, J. Gao, and Y.P. Sun, "Coaction of electric field induced strain and polarization effects in $\mathrm{La}_{0.7} \mathrm{Ca}_{0.3} \mathrm{MnO}_{3}$ /PMN-PT structures," Phys. Rev. B, vol. 79, no. 17, Art. No. 174437, 2009.

[162] A. Herklotz, A. D. Rata, L. Schultz, and K. Dörr, "Reversible strain effect on the magnetization of $\mathrm{LaCoO}_{3}$ films," Phys. Rev. B, vol. 79, no. 9, Art. No. 092409, 2009.

[163] M. Liu et al., "Giant Electric Field Tuning of Magnetic Properties in Multiferroic Ferrite/Ferroelectric Heterostructures," Adv. Funct. Mater., vol. 19, no. 11, pp. 1826-1831, 2009.

[164] J. J. Yang eta al., "Electric field manipulation of magnetization at room temperature in multiferroic $\mathrm{CoFe}_{2} \mathrm{O}_{4} / \mathrm{Pb}\left(\mathrm{Mg}_{1 / 3} \mathrm{Nb}_{2 / 3}\right)_{0.7} \mathrm{Ti}_{0.3} \mathrm{O}_{3}$ heterostructures," Appl. Phys. Lett., vol. 94, Art. No. 212504, 2009.

[165] J. H. Park et al., "In-plane strain control of the magnetic remanence and cation-charge redistribution in $\mathrm{CoFe}_{2} \mathrm{O}_{4}$ thin film grown on a piezoelectric substrate," Phys. Rev. B, vol. 81, no. 13, Art. No. 134401, 2010.

[166] J. H. Park, Y. K. Jeong, S. Ryu, J. Y. Son, H. M. Jang, " Electric-field-control of magnetic remanence of $\mathrm{NiFe}_{2} \mathrm{O}_{4}$ thin film epitaxially grown on $\mathrm{Pb}\left(\mathrm{Mg}_{1 / 3} \mathrm{Nb}_{2 / 3}\right) \mathrm{O}_{3}-\mathrm{PbTiO}_{3}$," Appl. Phys. Lett., vol. 96, Art. No. 192504, 2010.

[167] J. Lou, M. Liu, D. Reed, Y. Ren, N. X. Sun, "Giant Electric Field Tuning of Magnetism in Novel Multiferroic FeGaB/Lead Zinc Niobate-Lead Titanate (PZN-PT) Heterostructures," Adv. Mater., vol. 21, no. 46, p. 4711-4715, 2009.

[168] J-H. Kim, K-S. Ryu, J-W. Jeong, and S-C. Shin, "Large converse magnetoelectric coupling effect at room temperature in CoPd/PMN-PT (001) heterostructured," Appl. Phys. Lett., vol. 97, Art. No. 252508, 2010.

[169] T. Wu et al., "Electrical control of reversible and permanent magnetization reorientation for magnetoelectric memory devices," Appl. Phys. Lett., vol. 98, Art. No. 262504, 2011.

[170] C-J. Hsu, J. L. Hockel, and G. P. Carman, "Magnetoelectric manipulation of domain wall configuration in thin film Ni/[Pb( $\left.\left.\mathrm{Mn}_{1 / 3} \mathrm{Nb}_{2 / 3}\right) \mathrm{O}_{3}\right]_{0.68}\left[\mathrm{PbTiO}_{3}\right]_{0.32}$ (001) heterostructured," Appl. Phys. Lett., vol. 100, Art. No. 092902, 2012.

[171] M. Liu et al., "Voltage-Impulse-Induced Non-Volatile Ferroelastic Switching of Ferromagnetic Resonance for Reconfigurable Magnetoelectric Microwave Devices," Adv. Mater., vol. 25, no. 35, pp. 4886-4892, 2013. 
[172] B. Peng et al., "Deterministic Switching of Perpendicular Magnetic Anisotropy by Voltage Control of Spin Reorientation Transition in (Co/Pt) $3 / \mathrm{Pb}\left(\mathrm{Mg}_{1 / 3} \mathrm{Nb}_{2 / 3}\right) \mathrm{O}_{3}-\mathrm{PbTiO}_{3}$ Multiferroic Heterostructures," ACS Nano, vol. 11, no. 4, pp. 4337-4345, 2017.

[173] Q. Yang, T. Nan, Y. Zhang, Z. Zhou, B. Peng, W. Ren, Z.-G. Ye, N. X. Sun, and M. Liu, “Voltage control of perpendicular magnetic anisotropy in multi-ferroic (Co/Pt) $)_{3} / \mathrm{PbMg}_{1 / 3} \mathrm{Nb}_{2 / 3} \mathrm{O}_{3}-\mathrm{PbTiO}_{3}$ heterostructures," Phys. Rev. Appl., vol. 8, Art. No. 044006, 2017.

[174] M. Liu, J. Lou, S. Li, N. X. Sun, "E-Field Control of Exchange Bias and Deterministic Magnetization Switching in AFM/FM/FE Multiferroic Heterostructures," Adv. Funct. Mater., vol. 21, no. 13, pp. 2593-2598, 2011.

[175] D. E. Parkes et al., "Non-volatile voltage control of magnetization and magnetic domain walls in magnetostrictive epitaxial thin films," Appl. Phys. Lett., vol. 101, Art. No. 072402, 2012.

[176] [J. Wang et al., "Giant non-volatile magnetoelectric effects via growth anisotropy in $\mathrm{Co}_{40} \mathrm{Fe}_{40} \mathrm{~B}_{20}$ films on PMN-PT substrates," Appl. Phys. Lett., vol. 114, Art. No. 092401, 2019.

[177] J-M. Hu, Z. Li, L-Q. Chen, and C-W. Nan, "High-density magnetoresistive random access memory operating at ultralow voltage at room temperature," Nat. Commun., Vol. 2, Art. No. 553, 2011.

[178] J-M. Hu, Z. Li, L-Q. Chen, and C-W. Nan, "Design of a Voltage-Controlled Magnetic Random Access Memory Based on Anisotropic Magnetoresistance in a Single Magnetic Layer," Adv. Mater., vol. 24, pp. 2869-2873, 2012.

[179] Z. Wang, Y. Wang, W. Ge, J. Li, and D. Viehland, "Volatile and nonvolatile magnetic easyaxis rotation in epitaxial ferromagnetic thin films on ferroelectric single crystal substrates," Appl. Phys. Lett., vol. 103, Art. No. 132909, 2013.

[180] S. Zhang et al., "Electric-field control of nonvolatile magnetization in $\mathrm{CO}_{40} \mathrm{Fe}_{40} \mathrm{~B}_{20} / \mathrm{Pb}(\mathrm{Mg}(1 / 3) \mathrm{Nb}(2 / 3))(0.7) \mathrm{Ti}(0.3) \mathrm{O}_{3}$ structure at room temperature," Phys. Rev. Lett., Vol. 108, no. 13, Art. No. 137203, 2012.

[181] M. Ghidini et al., "Non-volatile electrically-driven repeatable magnetization reversal with no applied magnetic field," Nat. Commun., vol. 4, Art. No. 1453, 2013.

[182] K. Roy, S. Bandyopadhyay, and J. Atulasimha, "Binary switching in a 'symmetric' potential landscape," Sci. Rep., Vol. 3, Art. No. 3038, 2013.

[183] J.-M. Hu et al., "Purely Electric-Field-Driven Perpendicular Magnetization Reversal," Nano Lett., vol. 15, no. 1, pp. 616-622, 2015.

[184] J.-W. Lee, S.-C. Shin, S.-K. Kim, "Spin engineering of CoPd alloy films via the inverse piezoelectric effect," Appl. Phys. Lett., vol. 82, pp. 2458-2460, 2003.

[185] A. Brandlmaier et al., "In situ manipulation of magnetic anisotropy in magnetite thin films," Phys. Rev. B, vol. 77, Art. No. 104445, 2008.

[186] S. Q. Ren and M. Wuttig, "Magnetoelectric nano- $\mathrm{Fe}_{3} \mathrm{O}_{4} / \mathrm{CoFe}_{2} \mathrm{O}_{4} \| \mathrm{PbZr}_{0.53} \mathrm{Ti}_{0.47} \mathrm{O}_{3}$ composite," Appl. Phys. Lett., vol. 92, Art. No. 083502, 2008. 
[187] M. Liu, O. Obi, Z. H. Cai, J. Lou, G. M. Yang, K. S. Ziemer, and N. X. Sun, “Electrical tuning of magnetism in $\mathrm{Fe}_{3} \mathrm{O}_{4} / \mathrm{PZN}-\mathrm{PT}$ multiferroic heterostructures derived by reactive magnetron sputtering," J. Appl. Phys., Vol. 107, Art. No. 073916, 2010.

[188] T. H. E. Lahtinen, J. O. Tuomi, and S. van Dijken, "Pattern Transfer and Electric-Field-Induced Magnetic Domain Formation in Multiferroic Heterostructures," Adv. Mater., Vol. 23, no. 28, pp. 3187-3191, 2011.

[189] T. H. E. Lahtinen, K. J. A. Franke, and S. van Dijken, "Electric-field control of magnetic domain wall motion and local magnetization reversal," Sci. Rep., vol. 2, Art. No. 258, 2012.

[190] R. V. Chopdekar et al., "Spatially resolved strain-imprinted magnetic states in an artificial multiferroic," Phys. Rev. B, Vol. 86, Art. No. 014408, 2012.

[191] A. Casiraghi et al., "Influence of elastically pinned magnetic domain walls on magnetization reversal in multiferroic heterostructures," Phys. Rev. B, Vol. 92, Art. No. 054406, 2016.

[192] W. Eerenstein, M. Wiora, J. L. Prieto, J. F. Scott, and N. D. Mathur, "Giant sharp and persistent converse magnetoelectric effects in multiferroic epitaxial heterostructures," Nat. Mater., Vol. 6, pp. 348-351, 2007.

[193] S. Sahoo, S. Polisetty, C.-G. Duan, S. S. Jaswal, E. Y. Tsymbal, and C. Binek, "Ferroelectric control of magnetism in $\mathrm{BaTiO}_{3} / \mathrm{Fe}$ heterostructures via interface strain coupling," Phys. Rev. $B$, Vol. 76, Art. No. 092108, 2007.

[194] T. Taniyama, K. Akasaka, D. Fu, M. Itoh, H. Takashima, and B. Prijamboedi, “Electrical voltage manipulation of ferromagnetic microdomain structures in a ferromagnetic/ferroelectric hybrid structure," J. Appl. Phys., Vol. 101, no. 9, Art. No. 09F512, 2007.

[195] S. Brivio, D. Petti, R. Bertacco, and J. C. Cezar, "Electric field control of magnetic anisotropies and magnetic coercivity in $\mathrm{Fe}_{\mathrm{BaTiO}}$ (001) heterostructures," Appl. Phys. Lett., Vol. 98, no. 9, Art. No. 092505, 2011.

[196] G. Venkataiah, Y. Shirahata, M. Itoh, and T. Taniyama, "Manipulation of magnetic coercivity of Fe film in $\mathrm{Fe} \mathrm{BaTiO}_{3}$ heterostructure by electric field," Appl. Phys. Lett., Vol. 99, No. 10, Art. No. 102506 (2011).

[197] S. Geprägs, A. Brandlmaier, M. Opel, R. Gross, and S. T. B. Goennenwein, "Electric field controlled manipulation of the magnetization in $\mathrm{Ni} / \mathrm{BaTiO}_{3}$ hybrid structures," Appl. Phys. Lett. Vol. 96, No. 14, Art. No. 142509, 2010.

[198] M. Ghidini et al., "Perpendicular Local Magnetization Under Voltage Control in Ni Films on Ferroelectric $\mathrm{BaTiO}_{3}$ Substrates," Adv. Mater. Vol. 27, no. 8, pp. 1460-1465, 2015.

[199] Y. Shirahata et al., "Electric-field switching of perpendicularly magnetized multilayers," NPG Asia Mater. Vol. 7, Art. No. e198, 2015.

[200] S. Geprägs, D. Mannix, M. Opel, S. T. B. Goennenwein, and R. Gross, "Converse magnetoelectric effects in $\mathrm{Fe}_{3} \mathrm{O}_{4} / \mathrm{BaTiO}_{3}$ multiferroic híbrids," Phys. Rev. B Vol. 88, No. 5, Art. No. 054412, 2013. 
[201] T. Brintlinger et al., "In situ observation of reversible nanomagnetic switching induced by electric fields," Nano Lett., Vol. 10, No. 10, pp. 1219-1223, 2010.

[202] Y. Xie, Q. Zhan, Y. Liu, G. Dai, H. Yang, Z. Zuo, B. Chen, B. Wang, Y. Zhang, X. Rong, and R.W. Li, "Electric-field control of magnetic anisotropy in $\mathrm{Fe}_{81} \mathrm{Ga}_{19} / \mathrm{BaTiO}_{3}$ heterostructure films," AIP Adv., Vol. 4, Art. No. 117113, 2014.

[203] R. O. Cherifi et al., "Electric-field control of magnetic order above room temperature," Nat. Mater., Vol. 13, pp. 345-354, 2014.

[204] Z. Q. Liu et al., "Full Electroresistance Modulation in a Mixed-Phase Metallic Alloy," Phys. Rev. Lett., Vol. 116, No. 9, Art. No. 097203, 2016.

[205] J. Cui et al."Generation of localized strain in a thin film piezoelectric to control individual magnetoelectric heterostructures," Appl. Phys. Lett., Vol. 107, Art. No. 092903, 2015.

[206] X. Lu, Y. Kim, S. Goetze, X.-G. Li, S. Dong, P. Werner, M. Alexe, and D. Hesse, "Magnetoelectric Coupling in Ordered Arrays of Multilayered Heteroepitaxial $\mathrm{BaTiO}_{3} / \mathrm{CoFe}_{2} \mathrm{O}_{4}$ Nanodots," Nano Lett., Vol. 11, No. 8, pp. 3202-3206, 2011.

[207] G. Tian et al., "Magnetoelectric Coupling in Well-Ordered Epitaxial $\mathrm{BiFeO}_{3} / \mathrm{CoFe}_{2} \mathrm{O}_{4} / \mathrm{SrRuO}_{3}$ Heterostructured Nanodot Array," ACS Nano Vol. 10, No. 1, pp. 1025-1032, 2016.

[208] C.-G. Duan, S. S. Jaswal, and E. Y. Tsymbal, "Predicted Magnetoelectric Effect in Fe/BaTiO3 Multilayers: Ferroelectric Control of Magnetism," Phys. Rev. Lett. Vol. 97, Art. No. 047201, 2006.

[209] K. Yamauchi, B. Sanyal, and S. Picozzi, "Interface effects at a half-metal/ferroelectric junction," Appl. Phys. Lett. Vol. 91, No. 6, Art. No. 062506, 2007.

[210] M. Fechner, I. V. Maznichenko, S. Ostanin, A. Ernst, J. Henk, P. Bruno, and I. Mertig, "Magnetic phase transition in two-phase multiferroics predicted from first principles," Phys. Rev. B, Vol. 78, No. 21, Art. No. 212406, 2008.

[211] J. Lee, N. Sai, T. Cai, Q. Niu, and A. A. Demkov, "Interfacial magnetoelectric coupling in tricomponent superlattices," Phys. Rev. B, Vol. 81, No. 14, Art. No. 144425, 2010.

[212] J.-Q. Dai, Y.-M. Song, and H. Zhang, "Enhancement of magnetoelectric effect by combining different interfacial coupling mechanisms," J. Appl. Phys., Vol. 111, No. 11, Art. No. 114301, 2012.

[213] M. K. Niranjan, J. P. Velev, C.-G. Duan, S. S. Jaswal, and E. Y. Tsymbal, "Magnetoelectric effect at the $\mathrm{Fe}_{3} \mathrm{O}_{4} / \mathrm{BaTiO}_{3}$ (001) interface: A first-principles study," Phys. Rev. B, Vol. 78, No. 10, Art. No. 104405, 2008.

[214] V. S. Borisov, S. Ostanin, I. V. Maznichenko, A. Ernst, and I. Mertig, "Magnetoelectric properties of the $\mathrm{Co} / \mathrm{PbZr}_{x} \mathrm{Ti}_{1-\mathrm{x}} \mathrm{O}_{3}(001)$ interface studied from first principles," Phys. Rev. B, Vol. 89, No. 5, Art. No. 054436, 2014.

[215] J. D. Burton and E. Y. Tsymbal, "Prediction of electrically induced magnetic reconstruction at the manganite/ferroelectric interface," Phys. Rev. B, Vol. 80, No. 17, Art. No. 174406, 2009. 
[216] J. D. Burton and E. Y. Tsymbal, "Giant tunneling electroresistance effect driven by an electrically controlled spin valve at a complex oxide interface," Phys. Rev. Lett. Vol. 106, No. 15, Art. No. 157203, 2011.

[217] H. Chen et al., "Reversible modulation of orbital occupations via an interface-induced polar state in metallic manganites," Nano Lett., Vol. 14, No. 9, pp. 4965-4970, 2014.

[218] H. Lu et al., "Electric modulation of magnetization at the $\mathrm{BaTiO}_{3} / \mathrm{La}_{0.67} \mathrm{Sr}_{0.33} \mathrm{MnO}_{3}$ interfaces," Appl. Phys. Lett., Vol. 100, Art. No. 232904, 2012.

[219] X. Hong, A. Posadas, A. Lin, and C. H. Ahn, "Ferroelectric-field-induced tuning of magnetism in the colossal magnetoresistive oxide $\mathrm{La}_{1-\mathrm{x}} \mathrm{Sr}_{\mathrm{x}} \mathrm{MnO}_{3}$," Phys. Rev. B, Vol. 68, No. 13, Art. No. 134415, 2003.

[220] X. Hong, A. Posadas, and C. H. Ahn, "Examining the screening limit of field effect devices via the metal-insulator transition," Appl. Phys. Lett., Vol. 86, No. 14, Art. No. 142501, 2005.

[221] T. Kanki, H. Tanaka, and T. Kawai, "Electric control of room temperature ferromagnetism in a $\mathrm{Pb}\left(\mathrm{Zr}_{0.2} \mathrm{Ti}_{0.8}\right) \mathrm{O}_{3} / \mathrm{La}_{0.85} \mathrm{Ba}_{0.15} \mathrm{MnO} 3$ field-effect transistor," Appl. Phys. Lett., Vol. 89, No. 24, Art. No. 242506, 2006.

[222] H. J. A. Molegraaf, J. Hoffman, C. A. F. Vaz, S. Gariglio, D. van der Marel, C. H. Ahn, and J.M. Triscone, "Magnetoelectric Effects in Complex Oxides with Competing Ground States," Adv. Mater., Vol. 21, No. 34, pp. 3470-3474, 2009.

[223] C. A. F. Vaz et al., "Origin of the Magnetoelectric Coupling Effect in $\mathrm{Pb}\left(\mathrm{Zr}_{0.2} \mathrm{Ti}_{0.8}\right) \mathrm{O}_{3} / \mathrm{La}_{0.8} \mathrm{Sr}_{0.2} \mathrm{MnO}_{3}$ Multiferroic Heterostructures," Phys. Rev. Lett., Vol. 104, No. 12, Art. No. 127202, 2010.

[224] V. Garcia et al. "Ferroelectric Control of Spin Polarization," Science, Vol. 327, No. 5969, pp. 1106-1110, 201.)

[225] D. Pantel, S. Goetze, D. Hesse, and M. Alexe, "Reversible electrical switching of spin polarization in multiferroic tunnel junctions," Nat. Mater., Vol. 11, pp. 289-293, 2012.

[226] L. Bocher et al., "Atomic and Electronic Structure of the $\mathrm{BaTiO}_{3} /$ Fe Interface in Multiferroic Tunnel Junctions," Nano Lett., Vol. 12, No. 12, pp. 376-382, 2012.

[227] S. Valencia et al., "Interface-induced room-temperature multiferroicity in $\mathrm{BaTiO}_{3}$, " Nat. Mater., Vol. 10, pp. 753-758, 2011.

[228] J. P. Velev et al., "Magnetic tunnel junctions with ferroelectric barriers: prediction of four resistance states from first principles," Nano Lett., Vol. 9, No. 1, pp. 427-432, 2009.

[229] V. Laukhin et al., "Electric-Field Control of Exchange Bias in Multiferroic Epitaxial Heterostructures," Phys. Rev. Lett. 97, No. 22, Art. No. 227201, 2006.

[230] V. Skumryev et al., "Magnetization reversal by electric-field decoupling of magnetic and ferroelectric domain walls in multiferroic-based heterostructures," Phys. Rev. Lett., Vol. 106, No. 5, Art. No. 057206, 2011.

[231] L. W. Martin et al., "Nanoscale Control of Exchange Bias with $\mathrm{BiFeO}_{3}$ Thin Films," Nano Lett., vol. 8, no. 7, pp. 2050-2055, 2008. 
[232] H. Béa et al., "Mechanisms of Exchange Bias with Multiferroic $\mathrm{BiFeO}_{3}$ Epitaxial Thin Films," Phys. Rev. Lett., vol. 100, no. 1, Art. No. 017204, 2008.

[233] Y.-H. Chu et al., "Electric-field control of local ferromagnetism using a magnetoelectric multiferroic," Nat. Mater., vol. 7, pp. 478-482, 2008.

[234] S. M. Wu at al., "Reversible electric control of exchange bias in a multiferroic field-effect Device," Nat. Mater., vol. 9, pp. 756-761, 2010.

[235] J. T. Heron et al., "Electric-Field-Induced Magnetization Reversal in a FerromagnetMultiferroic Heterostructure," Phys. Rev. Lett., vol. 107, no. 21, Art. No. 217202, 2011.

[236] J. T. Heron, D. G. Schlom, and R. Ramesh, "Electric field control of magnetism using $\mathrm{BiFeO}_{3}$ based heterostructured," Appl. Phys. Rev., vol. 1, no. 2, Art. No. 021303, 2014.

[237] Z. Wang et al., "Magnetoelectric Assisted $180^{\circ}$ Magnetization Switching for Electric Field Addressable Writing in Magnetoresistive Random-Access Memory," ACS Nano, vol. 8, no. 8, pp. 7793-7800, 2014.

[238] J. J. Wang, J. M. Hu, J. Ma, J. X. Zhang, L. Q. Chen, and C. W. Nan, "Full $180^{\circ}$ Magnetization Reversal with Electric Fields," Sci. Rep., vol. 4, Art. No. 7507, 2014.

[239] R.-C. Peng, J. J. Wang, J.-M. Hu, L.-Q. Chen, and C.-W. Nan, "Electric-field-driven magnetization reversal in square-shaped nanomagnet-based multiferroic heterostructured," Appl. Phys. Lett., vol. 106, no. 14, Art. No. 142901, 2015.

[240] J. Cui, S. M. Keller, C.-Y. Liang, G. P. Carman, and C. S. Lynch, "Nanoscale magnetic ratchets based on shape anisotropy," Nanotechnology, vol. 28, no. 8, Art. No. 08LT01, 2017.

[241] N. D’Souza, M. Salehi Fashami, S. Bandyopadhyay, and J. Atulasimha, "Experimental Clocking of Nanomagnets with Strain for Ultralow Power Boolean Logic," Nano Lett., vol. 16, no. 2, pp. 1069-1075, 2016.

[242] V. Novosad et al., "Novel magnetostrictive memory Device," J. Appl. Phys., vol. 87, no. 9, p. 6400-6402, 2000.

[243] A. K. Biswas, S. Bandyopadhyay, and J. Atulasimha, "Energy-efficient magnetoelastic nonvolatile memory," Appl. Phys. Lett., vol. 104, no. 23, Art. No. 232403 , 2014.

[244] T. X. Nan et al., "A Strain-Mediated Magnetoelectric-Spin-Torque Hybrid Structure," Adv. Funct. Mater., Vol. 29, no. 6, Art. No. 1806371, 2019.

[245] N. Lei et al., "Strain-controlled magnetic domain wall propagation in hybrid piezoelectric/ferromagnetic structures," Nat. Commun., vol. 4, Art. No. 1378, 2013.

[246] E. de Ranieri et al., "Piezoelectric control of the mobility of a domain wall driven by adiabatic and non-adiabatic torques," Nat. Mater., vol. 12, pp. 808-814, 2013.

[247] K. J. A. Franke, B. Van de Wiele, Y. Shirahata, S. J. Hämäläinen, T. Taniyama, and S. van Dijken, "Reversible Electric-Field-Driven Magnetic Domain-Wall Motion," Phys. Rev. X, vol. 5, no. 1, Art. No. 011010, 2015. 
[248] D. López González et al., "Electric-field-driven domain wall dynamics in perpendicularly magnetized multilayers," AIP Adv., vol. 7, no. 3, Art. No. 035119, 2017.

[249] J. L. Hockel, A. Bur, T. Wu, K. P. Wetzlar, and G. P. Carman, "Electric field induced magnetization rotation in patterned $\mathrm{Ni}$ ring/ $\mathrm{Pb}\left(\mathrm{Mg}_{1 / 3} \mathrm{Nb}_{2 / 3}\right)_{3} \mathrm{O}_{(1-0.32)}-\left[\mathrm{PbTiO}_{3}\right]_{0.32}$ heterostructures," Appl. Phys. Lett., vol. 100, no. 2, Art. No. 022401, 2012.

[250] H. Sohn et al., "Electrically Driven Magnetic Domain Wall Rotation in Multiferroic Heterostructures to Manipulate Suspended On-Chip Magnetic Particles," ACS Nano, vol. 9, no. 5, pp. 4814-4826, 2015.

[251] R. P. Beardsley et al., "Deterministic control of magnetic vortex wall chirality by electric field," Sci. Rep., vol. 7, Art. No. 7613, 2017.

[252] M. Ghidini et al., "Voltage-driven annihilation and creation of magnetic vortices in Ni discs," Nanoscale, vol. 12, pp. 5652-5657, 2020.

[253] M. Ghidini et al., "Voltage-driven displacement of magnetic vortex cores," J. Phys D-Appl. Phys., vol. 53, no. 43, Art. No. 434003, 2020.

[254] Z. Li et al., "Strain-controlled skyrmion creation and propagation in ferroelectric/ ferromagnetic hybrid wires," J. Magn. Magn. Mater., vol. 455, pp. 19-24, 2018.

[255] R. Yanes et al., "Skyrmion motion induced by voltage-controlled in-plane strain gradients," Appl. Phys. Lett., vol. 115, no. 13, Art. No. 132401, 2019.

[256] J. Lou, M. Liu, D. Reed, Y. Ren, and N. X. Sun, "Giant Electric Field Tuning of Magnetism in Novel Multiferroic FeGaB/Lead Zinc Niobate-Lead Titanate (PZN-PT) Heterostructures," Adv. Mater., vol. 21, no. 46, pp. 4711-4715, 2009. (2009).

[257] Z. Zhou et al., "Probing electric field control of magnetism using ferromagnetic resonance," Nat. Commun., vol. 6, Art. No. 6082, 2015.

[258] A. V. Chumak, V. I. Vasyuchka, A. A. Serga, and B. Hillebrands, "Magnon spintronics", Nat. Phys., vol. 11, pp. 453-461, 2015.

[259] S. Cherepov et al., "Electric-field-induced spin wave generation using multiferroic magnetoelectric cells," Appl. Phys. Lett., vol. 104, no. 8, p. 082403, 2014.

[260] M. Balinskiy, A. C. Chavez, A. Barra, H. Chiang, G. P. Carman, and A. Khitun, "Magnetoelectric Spin Wave Modulator Based On Synthetic Multiferroic Structure," Sci. Rep., vol. 8, Art. No. 10867, 2018.

[261] A. V. Sadovnikov et al., "Magnon Straintronics: Reconfigurable Spin-Wave Routing in StrainControlled Bilateral Magnetic Stripes," Phys. Rev. Lett., Vol. 120, no. 25, Art. No. 257203, 2018.

[262] B. Van de Wiele, S. J. Hämäläinen, P. Baláz, F. Montoncello, and S. van Dijken, "Tunable short-wavelength spin wave excitation from pinned magnetic domain walls," Sci. Rep., vol. 6, Art. No. 21330, 2016.

[263] S. J. Hämäläinen, F. Brandl, K. J. A. Franke, D. Grundler, and S. van Dijken, "Tunable ShortWavelength Spin-Wave Emission and Confinement in Anisotropy-Modulated Multiferroic Heterostructures," Phys. Rev. Appl., vol. 8, no. 1, Art. No. 014020, 2017. 
[264] S. J. Hämäläinen, M. Madami, H. Qin, G. Gubbiotti, and S. van Dijken, “Control of spin-wave transmission by a programmable domain wall," Nat. Commun., vol. 9, Art. No. 4853, 2048.

[265] I. E. Dzyaloshinskii, "On the Magneto-Electrical Effect in Antiferromagnets," J. Expt. Theo. Phys., vol. 37, no. 3 p. 881, 1960.

[266] K. P. Jayachandran, J. M. Guedes, and H. C. Rodrigues, "Homogenization method for microscopic characterization of the composite magnetoelectric multiferroics," Sci. Rep., vol. 10, Art. No. 1276, 2020.

[267] E. Ascher, H. Rieder, H. Schmid, and H. Stössel, "Some properties of ferromagnetoelectric nickel-iodine boracite, $\mathrm{Ni}_{3} \mathrm{~B}_{7} \mathrm{O}_{13}$ l," J. Appl. Phys., vol. 37, no. 3, pp. 1404-1405, 1966.

[268] R. P. N. Santoro, "Survey of Magnetoelectric Materials," Massachusetts Institute of Technology, Massachusetts, USA1966.

[269] P. Martins and S. Lanceros-Méndez, "Polymer-based magnetoelectric materials," Adv. Funct. Mater., vol. 23, no. 27 pp. 3371-3385, 2013.

[270] P. Martins and S. Lanceros-Méndez, "Polymer-based magnetoelectric materials: To be or not to be," Appl. Mater. Today, vol. 15, pp. 558-561, 2019.

[271] C. S. Lehmann Fernández, N. Pereira, S. Lanceros-Méndez, and P. Martins, "Evaluation and optimization of the magnetoelectric response of $\mathrm{CoFe}_{2} \mathrm{O}_{4} /$ poly(vinylidene fluoride) composite spheres by computer simulation," Compos. Sci. Technol., vol. 146, pp. 119-130, 2017.

[272] C. S. Lehmann Fernández, N. Pereira, P. Martins, and S. Lanceros-Méndez, "Theoretical design of high-performance polymer-based magnetoelectric of fibrilar structures," Compos. Sci. Technol., vol. 155, pp. 126-136, 2018.

[273] N. Pereira, A. C. Lima, V. Correia, N. Perinka, S. Lanceros-Mendez, and P. Martins, "Magnetic proximity sensor based on magnetoelectric composites and printed coils," Materials, vol. 13, no. 7, Art. No. 7, 2020.

[274] R. Brito-Pereira, C. Ribeiro, S. Lanceros-Mendez, and P. Martins, "Magnetoelectric response on Terfenol-D/ P(VDF-TrFE) two-phase composites," Compos. B: Eng., vol. 120, no. pp. 97-102, 2017.

[275] R. Gonçalves, A. Larrea, T. Zheng, M. J. Higgins, V. Sebastian, S. Lanceros-Mendez, et al., "Synthesis of highly magnetostrictive nanostructures and their application in a polymer-based magnetoelectric sensing device," Eur. Polym. J., vol. 84, pp. 685-692, 2016.

[276] J. Gutiérrez, A. Lasheras, P. Martins, N. Pereira, J. M. Barandiarán, and S. Lanceros-Mendez, "Metallic glass/PVDF magnetoelectric laminates for resonant sensors and actuators: A review," Sensors (Switzerland), Vol. 17, no. 6, Art. No. 1251, 2017. 
[277] M. Silva, S. Reis, C. S. Lehmann, P. Martins, S. Lanceros-Mendez, A. Lasheras, et al., "Optimization of the magnetoelectric response of poly(vinylidene fluoride)/epoxy/vitrovac laminates," ACS Appl. Mater. Interfaces, vol. 5, no. 21, pp. 10912-10919, 2013.

[278] P. Martins, R. Gonçalves, S. Lanceros-Mendez, A. Lasheras, J. Gutiérrez, and J. M. Barandiarán, "Effect of filler dispersion and dispersion method on the piezoelectric and magnetoelectric response of $\mathrm{CoFe}_{2} \mathrm{O}_{4}$ /P(VDF-TrFE) nanocomposites," Appl. Surf. Sci., vol. 313, no. pp. 215-219, 2014.

[279] N. Pereira, A. C. Lima, S. Lanceros-Mendez, and P. Martins, "Magnetoelectrics: Three centuries of research heading towards the 4.0 industrial revolution," Materials, Vol. 13, no. 18, Art. No. 4033, 2020.

[280] M. P. Silva, P. Martins, A. Lasheras, J. Gutiérrez, J. M. Barandiarán, and S. Lanceros-Mendez, "Size effects on the magnetoelectric response on PVDF/Vitrovac 4040 laminate composites," J. Magn. Magn. Mater., vol. 377, no. pp. 29-33, 2015.

[281] P. Martins, A. C. Lopes, and S. Lanceros-Mendez, "Electroactive phases of poly(vinylidene fluoride): Determination, processing and applications," Prog. Polym. Sci., Vol. 39, no. 4, pp. 683706, 2014.

[282] P. Martins, M. Silva, and S. Lanceros-Mendez, "Determination of the magnetostrictive response of nanoparticles via magnetoelectric measurements," Nanoscale, Vol. 7, no. 21, pp. 9457-9461, 2015.

[283] S. Reis, M. P. Silva, N. Castro, V. Correia, J. G. Rocha, P. Martins, et al., "Electronic optimization for an energy harvesting system based on magnetoelectric Metglas/poly(vinylidene fluoride)/Metglas composites," Smart Mater. Struct., Vol. 25, no. 8, Art. No. 085028, 2016.

[284] S. Reis, M. P. Silva, N. Castro, V. Correia, J. Gutierrez, A. Lasheras, et al., "Optimized anisotropic magnetoelectric response of $\mathrm{Fe}_{61.6} \mathrm{Co}_{16.4} \mathrm{Si}_{10.8} \mathrm{~B}_{11.2} / \mathrm{PVDF} / \mathrm{Fe}_{61.6} \mathrm{Co}_{16.4} \mathrm{Si}_{10.8} \mathrm{~B}_{11.2}$ laminates for AC/DC magnetic field sensing," Smart Mater. Struct., vol. 25, no. 5, Art. No. 055050, 2016.

[285] P. Martins, J. S. Nunes, J. Oliveira, N. Peřinka, and S. Lanceros-Mendez, "Spray-printed magnetoelectric multifunctional composites," Compos. B Eng., Vol. 187, Art. No. 107829, 2020.

[286] A. C. Lima, N. Pereira, R. Policia, C. Ribeiro, V. Correia, S. Lanceros-Mendez, et al., "Allprinted multilayer materials with improved magnetoelectric response," J. Mater. Chem. C, Vol. 7, no. 18, pp. 5394-5400, 2019.

[287] A. Kulkarni, K. Meurisch, I. Teliban, R. Jahns, T. Strunskus, A. Piorra, et al., "Giant magnetoelectric effect at low frequencies in polymer-based thin film composites," Appl. Phys. Lett., Vol. 104, No. 2, Art. No. 022904, 2014. 
[288] Z. Fang, S. G. Lu, F. Li, S. Datta, Q. M. Zhang, and M. El Tahchi, "Enhancing the magnetoelectric response of Metglas/polyvinylidene fluoride laminates by exploiting the flux concentration effect," Appl. Phys. Lett., Vol. 95, no. 11, Art. No. 112903, 2009.

[289] S. Reis, N. Castro, M. P. Silva, V. Correia, J. G. Rocha, P. Martins, et al., "Fabrication and characterization of high-performance polymer-based magnetoelectric DC magnetic field sensors devices," IEEE Trans. Ind. Electron., Vol. 64, no. 6, pp. 4928-4934, 2017.

[290] M. Zeng, S. W. Or, and H. L. Wa Chan, "Magnetic field-induced strain and magnetoelectric effects in sandwich composite of ferromagnetic shape memory Ni-Mn-Ga crystal and piezoelectric PVDF polymer," IEEE Trans. Ultrason. Ferroelectr. Freq. Control., vol. 57, no. 10, pp. 2147-2153, 2010.

[291] M. Sadeghi, Y. Hojjat, and M. Khodaei, "Self-sensing feature of the ultrasonic nanodisplacement actuator in Metglas/PMN-PT/Metglas magnetoelectric composite," J. Mater. Sci.: Mat. Electron., Vol. 31, no. 1, pp. 740-751, 2020.

[292] A. Lasheras, J. Gutiérrez, S. Reis, D. Sousa, M. Silva, P. Martins, et al., "Energy harvesting device based on a metallic glass/PVDF magnetoelectric laminated composite," Smart Mater. Struct., vol. 24, no. 6, Art. No. 065024, 2015.

[293] S. Yuan, Y. Huang, J. Zhou, Q. Xu, C. Song, and G. Yuan, "A high-efficiency helical core for magnetic field energy harvesting," IEEE Trans. Power Electron., vol. 32, no. 7, pp. 5365-5376, 2017.

[294] P. Lu, D. Shang, J. Shen, Y. Chai, C. Yang, K. Zhai, et al., "Nonvolatile transtance change random access memory based on magnetoelectric P(VDF-TrFE)/Metglas heterostructures," Appl. Phys. Lett., Vol. 109, no. 25, Art. No. 252902, 2016.

[295] B. D. Truong, E. Andersen, C. Casados, and S. Roundy, "Magnetoelectric wireless power transfer for biomedical implants: Effects of non-uniform magnetic field, alignment and orientation," Sens. Actuator A Phys., vol. 316, Art. No. 112269, 2020.

[296] K. Malleron, A. Gensbittel, H. Talleb, and Z. Ren, "Experimental study of magnetoelectric transducers for power supply of small biomedical devices," Microelectron. J., vol. 88, pp. 184189, 2019.

[297] C. Ribeiro, V. Correia, P. Martins, F. M. Gama, and S. Lanceros-Mendez, "Proving the suitability of magnetoelectric stimuli for tissue engineering applications," Colloids Surf. B, vol. 140, pp. 430-436, 2016.

[298] C. Gong and X. Zhang, "Two-dimensional magnetic crystals and emergent heterostructure devices," Science, vol. 363, Art. No. 6428, 2019.

[299] H. Ohno et al., "Electric-field control of ferromagnetism," Nature, vol. 408, p. 944-946, 2000. 
[300] D. Chiba, M. Yamanouchi, F. Matsukura, and H. Ohno," Electrical Manipulation of Magnetization Reversal in a Ferromagnetic Semiconductor," Science, vol. 301, no. 5635, pp. 943945, 2003.

[301] M. Weisheit, S. Fähler, A. Marty, Y. Souche, C. Poinsignon, and D. Givord, "Electric FieldInduced Modification of Magnetism in Thin-Film Ferromagnets," Science, vol. 315, no. 5810, pp. 349-351, 2007.

[302] T. Maruyama et al., "Large voltage-induced magnetic anisotropy change in a few atomic layers of iron," Nat. Nanotech., vol. 4, pp. 158-161, 2009.

[303] D. Chiba, S. Fukami, K. Shimamura, N. Ishiwata, K. Kobayashi, and T. Ono, "Electrical control of the ferromagnetic phase transition in cobalt at room temperature," Nat. Mater., vol. 10, pp. 853-856, 2011.

[304] O. O. Brovko, P. Ruiz-Díaz, T. R. Dasa, and V. S. Stepanyuk, "Controlling magnetism on metal surfaces with non-magnetic means: electric fields and surface charging," J. Phys.: Condens. Matter., vol. 26, no. 9, Art. No. 093001, 2014.

[305] K. Leistner, "Electrochemical approaches to room temperature magnetoelectric materials," Curr. Opin. Electrochem., vol. 25, Art. No. 100636, 2021.

[306] A. Quintana et al., "Voltage-Induced Coercivity Reduction in Nanoporous Alloy Films: A Boost toward Energy-Efficient Magnetic Actuation," Adv. Funct. Mater., vol. 27, no. 32, Art. No. 1701904, 2017.

[307] C. Navarro-Senent et al., "Large magnetoelectric effects in electrodeposited nanoporous microdisks driven by effective surface charging and magneto-ionics," ACS Appl. Mater. Interfaces, vol. 10, no. 51, pp. 44897-44905, 2018.

[308] E. Dislaki, S. Robbennolt, M. Campoy-Quiles, J. Nogués, E. Pellicer, and J. Sort, "Coercivity Modulation in Fe-Cu Pseudo-Ordered Porous Thin Films Controlled by an Applied Voltage: A Sustainable, Energy-Efficient Approach to Magnetoelectrically Driven Materials," Adv. Sci., vol. 5, no. 8, Art. No. 1800499, 2018.

[309] K. Duschek, M. Uhlemann, H. Schlörb, K. Nielsch, and K. Leistner, "Electrochemical and in situ magnetic study of iron/iron oxide films oxidized and reduced in $\mathrm{KOH}$ solution for magnetoionic switching," Electrochem. Commun., vol. 72, pp. 153-156, 2016.

[310] K. Duschek, A. Petr, J. Zehner, K. Nielschn and K. Leistner, "All-electrochemical voltagecontrol of magnetization in metal oxide/metal nanoislands," J. Mater. Chem. C, vol. 6, pp. 84118417, 2018.

[311] A. Quintana et al., "Voltage-Controlled ON-OFF Ferromagnetism at Room Temperature in a Single Metal Oxide Film," ACS Nano, vol. 12, no. 10, pp. 10291-10300, 2018.

[312] S. Topolovec, P. Jerabek, D. V. Szabó, H. Krenn, and R. Würschum, „SQUID magnetometry combined with in situ cyclic voltammetry: A case study of tunable magnetism of $\gamma-\mathrm{Fe}_{2} \mathrm{O}_{3}$ nanoparticles," J. Magn. Magn. Mater., vol. 329, pp. 43-48, 2013. 
[313] C. Bi et al., Reversible Control of Co Magnetism by Voltage-Induced Oxidation,“ Phys. Rev. Lett., vol. 113, no. 26, Art. No. 267202, 2014.

[314] U. Bauer et al., "Magneto-ionic control of interfacial magnetism," Nat. Mater., vol. 14, pp. 174-181, 2015.

[315] D. A. Gilbert et al., "Structural and magnetic depth profiles of magneto-ionic heterostructures beyond the interface limit," Nat. Commun., vol. 7, Art. no. 12264, 2016.

[316] U. Bauer, S. Emori, and G. S. D. Beach, "Electric field control of domain wall propagation in Pt/Co/GdO films," Appl. Phys. Lett., vol. 100, no. 19, Art. No. 192408, 2012.

[317] Y. N. Yan et al., "Electrical control of Co/Ni magnetism adjacent to gate oxides with low oxygen ion mobility," Appl. Phys. Lett., vol. 107, no. 12, Art. No. 122407, 2015.

[318] J. de Rojas et al., "Boosting Room-Temperature Magneto-Ionics in a Non-Magnetic Oxide Semiconductor," Adv. Funct. Mater., vol. 30, no 36, Art. No. 2003704, 2020.

[319] J. de Rojas et al., "Voltage-driven motion of nitrogen ions: a new paradigm for magnetoionics," Nat. Commun., vol. 11, Art. no. 5871, 2020.

[320] K.-Y. Lee et al., "Fast Magneto-Ionic Switching of Interface Anisotropy Using YttriaStabilized Zirconia Gate Oxide," Nano Lett., vol. 20, no. 5, pp. 3435-3441, 2020.

[321] A. J. Tan et al., "Magneto-ionic control of magnetism using a solid-state proton pump," Nat. Mater., vol. 18, pp. 35-41, 2019.

[322] L. Baldrati, A. J. Tan, M. Mann, R. Bertacco, G. S. D. Beach, "Magneto-ionic effect in CoFeB thin films with in-plane and perpendicular-to-plane magnetic anisotropy," Appl. Phys. Lett., vol. 100, no. 1, Art. No. 012404, 2017.

[323] A. Quintana et al., "Tunable Magnetism in Nanoporous CuNi Alloys by Reversible VoltageDriven Element-Selective Redox Processes," Small, vol. 14, no. 21, Art. No. 1704396, 2018.

[324] N. Di, J. Kubal, Z. Zeng, J. Greeley, F. Maroun, and P. Allongue, "Influence of controlled surface oxidation on the magnetic anisotropy of Co ultrathin films," Appl. Phys. Lett., vol. 106, no. 12, Art. No. 122405, 2015.

[325] S. Ghosh, "Switching magnetic order in nanoporous Pd-Ni by electrochemical charging," J. Mater. Res., vol. 28, no. 21, pp. 3010-3017, 2013.

[326] K. Duschek, D. Pohl, S. Fähler, K. Nielsch, and K. Leistner, "Research Update: Magnetoionic control of magnetization and anisotropy in layered oxide/metal heterostructures," APL Mater., vol. 4, no. 3, Art. No. 032301, 2016.

[327] S. Robbennolt et al., "Electric Field Control of Magnetism in Iron Oxide Nanoporous Thin Films," ACS Appl. Mater. Interf., vol. 11, no. 46, pp. 37338-37346, 2019.

[328] M. Cialone, A. Nicolenco, S. Robbennolt, E. Menéndez, G. Rius, J. Sort, "Voltage-Induced ON Switching of Magnetism in Ordered Arrays of Non-Ferrimagnetic Nanoporous Iron Oxide Microdisks," Adv. Mater. Interfaces, vol. 8, no. 1, Art. No. 2001143, 2021. 
[329] S. Robbennolt et al., "Reversible, Electric-Field Induced Magneto-lonic Control of Magnetism in Mesoporous Cobalt Ferrite Thin Films," Sci. Rep., vol. 9, Art. No. 10804, 2019.

[330] Q. Zhang et al., "Lithium-Ion Battery Cycling for Magnetism Control," Nano Lett., vol. 16, no. 1, pp. 583-587, 2016.

[331] S. Dasgupta et al., "Toward On-and-Off Magnetism: Reversible Electrochemistry to Control Magnetic Phase Transitions in Spinel Ferrites," Adv. Funct. Mater., vol. 26, no. 41, pp. 7507-7515, 2016.

[332] G. Wei et al., "Reversible control of the magnetization of spinel ferrites based electrodes by lithium-ion migration," Sci. Rep., vol. 7, Art. No. 12554, 2017.

[333] G. Wei et al., "Reversible control of magnetization of $\mathrm{Fe}_{3} \mathrm{O}_{4}$ by a solid-state film lithium battery," Appl. Phys. Lett., vol. 110, no. 6, Art. No. 062404, 2017.

[334] S. Vasala et al., "Reversible Tuning of Magnetization in a Ferromagnetic RuddlesdenPopper-Type Manganite by Electrochemical Fluoride-Ion Intercalation," Adv. Electron. Mater., vol. 6, no. 2, p.1900974, 2020.

[335] F. Ibrahim, A. Hallal, B. Dieny, M. Chshiev, "Establishing characteristic behavior of voltage control of magnetic anisotropy by ionic migration," Phys. Rev. B, vol. 98, no. 21, Art. No. 214441, 2018.

[336] C. Navarro-Senent et al., "Enhancing Magneto-Ionic Effects in Magnetic Nanostructured Films via Conformal Deposition of Nanolayers with Oxygen Acceptor/Donor Capabilities," ACS Appl. Mater. Interfaces, vol. 12, no. 12, pp. 14484-14494, 2020.

[337] S. Robbennolt, P. Wu, A. Nicolenco, P. Mercier-Fernández, M. Coll, J. Sort, "Magneto-ionic control of magnetism in two-oxide nanocomposite thin films comprising mesoporous cobalt ferrite conformally nanocoated with $\mathrm{HfO}_{2}$," Nanoscale, vol. 12, pp. 5987-5994, 2020.

[338] F. Maroun et al., "Potential dependence of the structure and magnetism of electrodeposited Pd/Co/Au(111) layers," J. Electroanal. Chem., vol. 819, p. 322, 2018.

[339] X. Ye et al., "Giant voltage-induced modification of magnetism in micron-scale ferromagnetic metals by hydrogen charging," Nat. Commun., vol. 11, Art. No. 4849, 2020.

[340] X. Ye et al., "Magnetoelectric Tuning of Pinning-Type Permanent Magnets through AtomicScale Engineering of Grain Boundaries," Adv. Mater., vol. 33, no. 5, Art. No. 2006853, 2021.

[341] P.-J. Hsu et al., "Inducing skyrmions in ultrathin Fe films by hydrogen exposure," Nat. Commun., vol. 9, Art. No. 1571, 2018.

[342] M. Gößler, M. Albu, G. Klinser, E. Steyskal, H. Krenn, R. Würschum, "Magneto-ionic switching of superparamagnetism," Small, vol. 15, no. 46, p. 1904523, 2019.

[343] N. Lu et al., "Electric-field control of tri-state phase transformation with a selective dualion switch," Nature, vol. 546, pp.124-128, 2017.

[344] S. Shen et al., "Manipulate the electronic and magnetic states in $\mathrm{NiCO}_{2} \mathrm{O}_{4}$ films through electric-field induced protonation at elevated temperature," Adv. Mater., vol. 31, no. 16, p. 1900458, 2019. 
[345] X. Zhou, Y. Yan, M. Jiang, B. Cui, F. Pan, and C. Song, "Role of Oxygen lon Migration in the Electrical Control of Magnetism in Pt/Co/Ni/HfO 2 Films," J. Phys. Chem. C, vol. 120, no. 3, pp. 1633-1639, 2016.

[346] F. N. Tan et al., "Electric field control on gated $\mathrm{Pt} / \mathrm{Co} / \mathrm{SiO}_{2}$ heterostructure with insulating polymer," J. Phys. D: Appl. Phys., vol. 51, no. 36, Art. No. 365001, 2018.

[347] K. Leistner et al., "Electrode processes and in situ magnetic measurements of FePt films in a LiPF 6 based electrolyte," Electrochim. Acta, vol. 81, pp. 330-337, 2012.

[348] K. Leistner et al., "Electric-field control of magnetism by reversible surface reduction and oxidation reactions," Phys. Rev. B, vol. 87, no. 22, Art. No. 224411, 2013.

[349] S. Dasgupta et al., "Intercalation-Driven Reversible Control of Magnetism in Bulk Ferromagnets," Adv. Mater., vol. 26, no. 27, pp. 4639-4644, 2014.

[350] L. A. Dubraja et al., "Electrochemical Tuning of Magnetism in Ordered Mesoporous Transition-Metal Ferrite Films for Micromagnetic Actuation," ACS Appl. Nano Mater., vol. 1, no. 1, pp. 65-72, 2018.

[351] C. Reitz, C. Suchomski, D. Wang, H. Hahnad, and T. Brezesinski, "In situ tuning of magnetization via topotactic lithium insertion in ordered mesoporous lithium ferrite thin films," J. Mater. Chem. C, vol. 4, pp. 8889-8896, 2016.

[352] S. Robbennolt, A. Quintana, E. Pellicer, and J. Sort, "Large magnetoelectric effects mediated by electric-field-driven nanoscale phase transformations in sputtered (nanoparticulate) and electrochemically dealloyed (nanoporous) Fe-Cu films," Nanoscale, vol. 10, pp. 14570-14578, 2018.

[353] J. L. Volakis, C.-C. Chen, and K. Fujimoto, Small antennas: miniaturization techniques \& applications. McGraw-Hill, 2010.

[354] D. B. Miron, Small antenna design. Elsevier, 2006.

[355] Z. Yao, Y. E. Wang, S. Keller, G. P. J. I. T. o. A. Carman, and Propagation, "Bulk acoustic wavemediated multiferroic antennas: Architecture and performance bound," IEEE Trans. Antennas Propag., vol. 63, no. 8, pp. 3335-3344, 2015.

[356] J. P. Domann and G. P. J. J. o. A. P. Carman, "Strain powered antennas," J. Appl. Phys., vol. 121, no. 4, Art. No. 044905, 2017.

[357] X. Liang, H. Chen, N. Sun, H. Lin, Y. Gao, and N. X. Sun, "Mechanically driven SMR-based MEMS magnetoelectric antennas," 2020 IEEE Antenna Propagation Symposium, In Publishing 2020.

[358] M. Zaeimbashi et al., "NanoNeuroRFID: A wireless implantable device based on magnetoelectric antennas," IEEE J. Electromagn., RF, Microw. Med. Biol., vol. 3, no. 3, pp. 206215, 2019.

[359] M. Zaeimbashi et al., "Ultra-compact Dual-band Smart NEMS Magnetoelectric Antennas for Simultaneous Wireless Energy Harvesting and Magnetic Field Sensing," biorxiv. 2020. 
[360] H. Chen, X. Liang, N. Sun, H. Lin, Y. Gao, and N. X. Sun, "An Ultra-Compact ME Antenna Design for Implantable Wireless Communication," 2020 IEEE Antenna Propagation Symposium, In Publishing 2020.

[361] J. Lenz and S. Edelstein, "Magnetic sensors and their applications," IEEE Sensors J., vol. 6, no. 3, pp. 631-649, 2006.

[362] S. Dong, J. Zhai, J. Li, and D. Viehland, "Near-ideal magnetoelectricity in high-permeability magnetostrictive/piezofiber laminates with a (2-1) connectivity," Appl. Phys. Lett., vol. 89, no. 25, Art. No. 252904, 2006.

[363] S. M. Gillette, A. L. Geiler, D. Gray, D. Viehland, C. Vittoria, and V. G. Harris, "Improved Sensitivity and Noise in Magneto-Electric Magnetic Field Sensors by Use of Modulated AC Magnetostriction," IEEE Magn. Lett., vol. 2, pp. 2500104-2500104, 2011.

[364] L. Shen et al., "Magnetoelectric nonlinearity in magnetoelectric laminate sensors," J. Appl. Phys., vol. 110, no. 11, Art. No. 114510, 2011.

[365] Y. Shen, J. Gao, Y. Wang, P. Finkel, J. Li, and D. Viehland, "Piezomagnetic strain-dependent non-linear magnetoelectric response enhancement by flux concentration effect," Appl. Phys. Lett., vol. 102, no. 17, Art. No. 172904, 2013.

[366] Y. Liu et al., "Frequency conversion in magnetoelectric composites for quasi-static magnetic field detection," Appl. Phys. Lett., vol. 103, no. 21, Art. No. 212902, 2013.

[367] M. M. Vopson, "Fundamentals of multiferroic materials and their possible applications," Crit. Rev. Solid State Mater. Sci., vol. 40, no. 4, pp. 223-250, 2015.

[368] J. Van Suchtelen, "Product properties: a new application of composite materials," Philips Res. Re.p, vol. 27, no. 1, pp. 28-37, 1972.

[369] R. Jahns, H. Greve, E. Woltermann, E. Quandt, and R. Knöchel, "Sensitivity enhancement of magnetoelectric sensors through frequency-conversion," Sens. Actuator A Phys., vol. 183, pp. 1621, 2012.

[370] S. Marauska, R. Jahns, H. Greve, E. Quandt, R. Knöchel, and B. Wagner, "MEMS magnetic field sensor based on magnetoelectric composites," J. Micromech. Microeng., vol. 22, no. 6, Art. No. 065024, 2012.

[371] S. Fichtner, T. Reimer, S. Chemnitz, F. Lofink, and B. Wagner, "Stress controlled pulsed direct current co-sputtered $\mathrm{Al}_{1-\mathrm{x}} \mathrm{Sc}_{\mathrm{x}} \mathrm{N}$ as piezoelectric phase for micromechanical sensor applications," APL Materials, vol. 3, no. 11, Art. No. 116102, 2015.

[372] J. Su et al., "AIScN-based MEMS magnetoelectric sensor," Appl. Phys. Lett., vol. 117, no. 13, Art. No. 132903, 2020.

[373] V. Röbisch et al., "Pushing the detection limit of thin film magnetoelectric heterostructures," J. Mater. Res., vol. 32, no. 6, pp. 1009-1019, 2017.

[374] P. Hayes et al., "Electrically modulated magnetoelectric sensors," Appl. Phys. Lett., vol. 108, no. 18, Art. No. 182902, 2016. 
[375] A. Kittmann et al., "Wide band low noise love wave magnetic field sensor system," Sci. Rep., vol. 8, Art. No. 278, 2018.

[376] H. Mishra et al., "Temperature compensated magnetic field sensor based on love waves," Smart Mater. Struct., vol. 29, no. 4, Art. No. 045036, 2020.

[377] A. Ludwig and E. Quandt, "Optimization of the Delta-E effect in thin films and multilayers by magnetic field annealing," IEEE Trans. Magn., vol. 38, no. 5, pp. 2829-2831, 2002.

[378] C. Dong et al., "Characterization of magnetomechanical properties in FeGaB thin films," Appl. Phys. Lett.s, vol. 113, no. 26, Art. No. 262401, 2018.

[379] X. Liang et al., "Soft Magnetism, Magnetostriction, and Microwave Properties of Fe-Ga-C Alloy Films," IEEE Magn. Lett., vol. 10, pp. 1-5, 2018.

[380] J. Wang et al., "Magnetostriction, Soft Magnetism, and Microwave Properties in Co-Fe-C Alloy Films," Phys.I Rev. Appl., vol. 12, no. 3, Art. No. 034011, 2019.

[381] E. Lage et al., "Exchange biasing of magnetoelectric composites," Nat. Mater., vol. 11, no. 6, pp. 523-529, 2012.

[382] V. Röbisch et al., "Exchange biased magnetoelectric composites for magnetic field sensor application by frequency conversion," J. Appl. Phys., vol. 117, no. 17, Art. No. 17B513, 2015.

[383] E. Lage, F. Woltering, E. Quandt, and D. Meyners, "Exchange biased magnetoelectric composites for vector field magnetometers," J. Appl. Phys., vol. 113, no. 17, Art. No. 17C725, 2013.

[384] E. Lage et al., "Magnetic domain control and voltage response of exchange biased magnetoelectric composites," Appl. Phys. Lett., vol. 104, no. 13, Art. No. 132405, 2014.

[385] N. O. Urs, I. Teliban, A. Piorra, R. Knöchel, E. Quandt, and J. McCord, "Origin of hysteretic magnetoelastic behavior in magnetoelectric 2-2 composites," Appl. Phys. Lett., vol. 105, no. 20, Art. No. 202406, 2014.

[386] N. O. Urs et al., "Direct Link between Specific Magnetic Domain Activities and Magnetic Noise in Modulated Magnetoelectric Sensors," Phys. Rev. Appl., vol. 13, no. 2, Art. No. 024018, 2020.

[387] M. Jovičević Klug et al., "Antiparallel exchange biased multilayers for low magnetic noise magnetic field sensors," Appl. Phys. Lett., vol. 114, no. 19, Art. No. 192410, 2019.

[388] S. Zabel et al., "Multimode delta-E effect magnetic field sensors with adapted electrodes," Appl. Phys. Lett., vol. 108, no. 22, Art. No. 222401, 2016.

[389] J. Schmalz, M. C. Krantz, A. Knies, H. Lüder, and M. Gerken, "Signal-to-noise ratio enhanced electrode configurations for magnetoelectric cantilever sensors," AIP Adv., vol. 10, no. 7, Art. No. 075314, 2020.

[390] P. Durdaut et al., "Thermal-mechanical noise in resonant thin-film magnetoelectric sensors," IEEE Sensors J., vol. 17, no. 8, pp. 2338-2348, 2017. 
[391] S. Gillette, A. Geiler, D. Gray, D. Viehland, C. Vittoria, and V. Harris, "Improved sensitivity and noise in magneto-electric magnetic field sensors by use of modulated AC magnetostriction," IEEE Magn. Lett.s, vol. 2, pp. 2500104-2500104, 2011.

[392] S. Salzer et al., "Noise limits in thin-film magnetoelectric sensors with magnetic frequency conversion," IEEE Sensors J., vol. 18, no. 2, pp. 596-604, 2017.

[393] Y. Shen, D. Ma, and J. Gao, "A Man-Portable Magnetoelectric DC Magnetic Sensor With Extremely High Sensitivity," IEEE Electron Dev. Lett., vol. 39, no. 9, pp. 1417-1420, 2018.

[394] Y. K. Fetisov, D. A. Burdin, D. V. Chashin, and N. A. Ekonomov, "High-sensitivity wideband magnetic field sensor using nonlinear resonance magnetoelectric effect," IEEE Sensors J., vol. 14, no. 7, pp. 2252-2256, 2014.

[395] X. Zhuang et al., "Mechanical noise limit of a strain-coupled magneto (elasto) electric sensor operating under a magnetic or an electric field modulation," IEEE Sensors J., vol. 15, no. 3, pp. 1575-1587, 2014.

[396] J. McCord, R. Schäfer, M. Frommberger, S. Glasmachers, and E. Quandt, "Stress-induced remagnetization in magnetostrictive films," J. Appl. Phys., vol. 95, no. 11, pp. 6861-6863, 2004.

[397] R. D. Shull, E. Quandt, A. Shapiro, S. Glasmachers, and M. Wuttig, "Magneto-optic indicator film observations of domain motion in magnetostrictive materials under stress," J. Appl. Phys., vol. 95, no. 11, pp. 6948-6950, 2004.

[398] J. McCord, "Irregular domain patterns in structured magnetic thick films," J. Appl. Phys., vol. 95, no. 11, pp. 6855-6857, 2004.

[399] M. Staruch, M.-T. Yang, J. Li, C. Dolabdjian, D. Viehland, and P. Finkel, "Frequency reconfigurable phase modulated magnetoelectric sensors using $\Delta \mathrm{E}$ effect," Appl. Phys. Lett., vol. 111, no. 3, Art. No. 032905, 2017.

[400] Y. K. Fetisov et al., "Bistability in a multiferroic composite resonator," Appl. Phys. Lett., vol. 113, no. 2, Art. No. 022903, 2018.

[401] P. Hayes et al., "Converse Magnetoelectric Composite Resonator for Sensing Small Magnetic Fields," Sci. Rep., vol. 9, no. 1, pp. 1-10, 2019.

[402] A. Ganguly, K. Davis, D. Webb, C. Vittoria, and D. Forester, "Magnetically tuned surfaceacoustic-wave phase shifter," Electron. Lett., vol. 11, no. 25, pp. 610-611, 1975.

[403] M. Kadota, S. Ito, Y. Ito, T. Hada, and K. Okaguchi, "Magnetic sensor based on surface acoustic wave resonators," Jap. J. Appl. Phys., vol. 50, no. 7S, Art. No. 07HD07, 2011.

[404] H. Zhou, A. Talbi, N. Tiercelin, and O. Bou Matar, "Multilayer magnetostrictive structure based surface acoustic wave devices," Appl. Phys. Lett., vol. 104, no. 11, Art. No. 114101, 2014.

[405] V. Polewczyk et al., "Unipolar and bipolar high-magnetic-field sensors based on surface acoustic wave resonators," Phys. Rev. Appl., vol. 8, no. 2, Art. No. 024001, 2017.

[406] W. Wang, Y. Jia, X. Xue, Y. Liang, and Z. Du, "Grating-patterned FeCo coated surface acoustic wave device for sensing magnetic field," AIP Adv., vol. 8, no. 1, Art. No. 015134, 2018. 
[407] X. Liu et al., "Influence of the delta-E effect on a surface acoustic wave resonator," Appl. Phys. Lett., vol. 114, no. 6, Art. No.. 062903, 2019.

[408] X. Liu et al., "Self-biased vector magnetic sensor based on a Love-type surface acoustic wave resonator," Appl. Phys. Lett., vol. 113, no. 8, v. 082402, 2018.

[409] A. Mazzamurro et al., "Giant Magnetoelastic Coupling in a Love Acoustic Waveguide Based on Tb Co 2/Fe Co Nanostructured Film on ST-Cut Quartz," Phys. Rev. Appl., vol. 13, no. 4, Art. No. 044001, 2020.

[410] V. Schell et al., "Magnetic anisotropy controlled FeCoSiB thin films for surface acoustic wave magnetic field sensors," Appl. Phys. Lett., vol. 116, no. 7, Art. No. 073503, 2020.

[411] J. Labrenz et al., "Frequency Response of SAW Delay Line Magnetic Field/Current Sensor," IEEE Sensors Lett.s, vol. 3, no. 10, pp. 1-4, 2019.

[412] J. Schmalz et al., "Multi-Mode Love-Wave SAW Magnetic-Field Sensors," Sensors, vol. 20, no. 12, Art. No. 3421, 2020.

[413] A. Kittmann et al., "Sensitivity and Noise Analysis of SAW Magnetic Field Sensors with varied Magnetostrictive Layer Thicknesses," Sens. Actuator A Phys., Art. No. 111998, 2020.

[414] P. Durdaut et al., "Fundamental Noise Limits and Sensitivity of Piezoelectrically Driven Magnetoelastic Cantilevers," J. Microelectromech. Syst., vol. 29, no. 5, pp. 1347-1361, 2020.

[415] P. Durdaut et al., "Noise analysis and comparison of phase-and frequency-detecting readout systems: Application to SAW delay line magnetic field sensor," IEEE Sensors J., vol. 19, no. 18, pp. 8000-8008, 2019.

[416] C. Tsang and S. Decker, "The origin of Barkhausen noise in small permalloy magnetoresistive sensors," J. Appl. Phys., vol. 52, no. 3, pp. 2465-2467, 1981.

[417] C. Tsang, "Magnetics of small magnetoresistive sensors," J. Appl. Phys., vol. 55, no. 6, pp. 2226-2231, 1984.

[418] P. Stephanou and A. Pisano, "PS-4 GHZ contour extensional mode aluminum nitride MEMS resonators," in 2006 IEEE Ultrasonics Symposium, 2006, pp. 2401-2404: IEEE.

[419] G. Piazza, P. J. Stephanou, and A. P. Pisano, "Piezoelectric aluminum nitride vibrating contour-mode MEMS resonators," Departmental Papers (ESE), p. 223, 2006.

[420] M. Rinaldi, C. Zuniga, and G. Piazza, "Ultra-thin-film AIN contour-mode resonators for sensing applications," in 2009 IEEE International Ultrasonics Symposium, 2009, pp. 714-717: IEEE.

[421] M. Rinaldi, C. Zuniga, C. Zuo, and G. Piazza, "Super-high-frequency two-port AIN contourmode resonators for RF applications," IEEE Trans. Ultrason. Ferroelectr. Freq. Control, vol. 57, no. 1, pp. 38-45, 2009.

[422] J. Lou, R. Insignares, Z. Cai, K. S. Ziemer, M. Liu, and N. X. Sun, "Soft magnetism, magnetostriction, and microwave properties of FeGaB thin films," Appl. Phys. Lett., vol. 91, no. 18, Art. No. 182504, 2007. 
[423] J. Ryu, A. V. Carazo, K. Uchino, and H.-E. Kim, "Magnetoelectric properties in piezoelectric and magnetostrictive laminate composites," Jap. J. Appl. Phys., vol. 40, no. 8R, pp. 4948-4951, 2001.

[424] G. Piazza, O. Brand, I. Dufour, S. Heinrich, and F. Josse, "Piezoelectric resonant MEMS," Resonant MEMS: Wiley Online Library, 2015, pp. 147-172.

[425] Y. Hui, T. Nan, N. X. Sun, and M. Rinaldi, "High resolution magnetometer based on a high frequency magnetoelectric MEMS-CMOS oscillator," J. Microelectromech. Syst., vol. 24, no. 1, pp. 134-143, 2014.

[426] M. Li et al., "Ultra-sensitive NEMS magnetoelectric sensor for picotesla DC magnetic field detection," Appl. Phys. Lett., vol. 110, no. 14, Art. No. 143510, 2017.

[427] M. Zaeimbashi et al., "Ultra-compact dual-band smart NEMS magnetoelectric antennas for simultaneous wireless energy harvesting and magnetic field sensing," bioRxiv, 2020.

[428] M. Rinaldi, C. Zuo, J. Van der Spiegel, and G. Piazza, "Reconfigurable CMOS oscillator based on multifrequency AIN contour-mode MEMS resonators," IEEE Trans. Electron Dev., vol. 58, no. 5, pp. 1281-1286, 2011.

[429] G. Herzer, "Magnetomechanical damping in amorphous ribbons with uniaxial anisotropy," Mater. Sci. Eng. A, vol. 226, pp. 631-635, 1997.

[430] G. Herzer, "Effect of domain size on the magneto-elastic damping in amorphous ferromagnetic metals: Dedicated to Professor Dr. Helmut Kronmüller on the occasion of his 70th birthday," Zeitschrift für Metallkunde, vol. 93, no. 10, pp. 978-982, 2002.

[431] B. Berry and W. Pritchet, "Vibrating reed internal friction apparatus for films and foils," IBM J. Res. Develop., vol. 19, no. 4, pp. 334-343, 1975.

[432] E. Klokholm, "The measurement of magnetostriction in ferromagnetic thin films," IEEE Trans. Magn., vol. 12, no. 6, pp. 819-821, 1976.

[433] K. Kawakami, S. Narishige, and M. Takagi, "A high frequency permeance meter for anisotropic films and its application in the determination of magneto striction constants IEEE Trans. Magn., vol. 19, no. 5, pp. 2154-2156, 1983.

[434] A. C. Tam and H. Schroeder, "A new high-precision optical technique to measure magnetostriction of a thin magnetic film deposited on a substrate," IEEE Trans. Magn., vol. 25, no. 3, pp. 2629-2638, 1989.

[435] T. Shima and H. Fujimori, "An accurate measurement of magnetostriction of thin films by using nano-indentation system," IEEE Trans. Magn., vol. 35, no. 5, pp. 3832-3834, 1999.

[436] M. Rinaldi and G. Piazza, "Effects of volume and frequency scaling in AIN contour mode NEMS resonators on oscillator phase noise," in 2011 Joint Conference of the IEEE International Frequency Control and the European Frequency and Time Forum (FCS) Proceedings, 2011, pp. 15. 
[437] Y. Hui and M. Rinaldi, "Fast and high resolution thermal detector based on an aluminum nitride piezoelectric microelectromechanical resonator with an integrated suspended heat absorbing element," Appl. Phys. Lett., vol. 102, no. 9, Art. No. 093501, 2013.

[438] K.-N. Tu, Electronic thin-film reliability. Cambridge University Press, 2010.

[439] S. Timoshenko, "Analysis of bi-metal thermostats," Josa, vol. 11, no. 3, pp. 233-255, 1925.

[440] H. Mehner, S. Leopold, and M. Hoffmann, "Variation of the intrinsic stress gradient in thin aluminum nitride films," J. Micromech. Microeng., vol. 23, no. 9, Art. No. 095030, 2013.

[441] S. Choi and B. A. Griffin, "Local residual stress monitoring of aluminum nitride MEMS using UV micro-Raman spectroscopy," J. Micromech. Microeng., vol. 26, no. 2, Art. No. 025009, 2016.

[442] M. D. Henry, R. Timon, T. R. Young, C. Nordquist, and B. Griffin, "AIN and ScAIN contour mode resonators for RF filters," ECS Trans., vol. 77, no. 6, pp. 23-32, 2017.

[443] S. Urazhdin et al., "Nanomagnonic devices based on the spin-transfer torque," Nat. Nanotechnol., Vol. 9, pp. 509-513, 2014.

[444] B. Dieny et al., "Spin-transfer effect and its use in spintronic components," Int. J. Nanotechnol., Vol. 7, pp. 591-614, 2010.

[445] H. Cai et al., "Spin-transfer effect and its use in spintronic components," Appl. Sci., Vol. 7, no. 9, Art. No. 929, 2017.

[446] K. Jabeur, et al., "Study of spin transfer torque (STT) and spin orbit torque (SOT) magnetic turnnel juntions (MTJS) at advanced CMOS technology nodes," E.L.E.L.I.J, Vol. 6, no. 1, pp. 1-9, 2017.

[447] J. M. Hu and C.-W. Nan., "Opportunities and challenges for magnetoelectric devices," APL Mater., Vol. 7, Art. No. 080905,2019.

[448] K. L. Wang, H. Lee and P. K. Amiri, "Magnetoelectric Random Access Memory-Based Circuit Design by Using Voltage-Controlled Magnetic Anisotropy in Magnetic Tunnel Junctions," IEEE Trans. Nanotechnol., Vol. 14, pp. 992-997, 2015.

[449] S Bhatti, R Sbiaa, A Hirohata, H Ohno, S Fukami and S.N. Piramanayagam, "Spintronics based random access memory: a review," Mater. Today, Vol. 20, no. 9, pp 530-548, 2017.

[450] I.L. Prejbeanu et al., "Thermally assisted MRAM", J. Phys.: Condens. Matter, Vol. 19, Art. No. 165218, 2007.

[451] Yiming Huai, "Spin-transfer torque MRAM (STT-MRAM): Challenges and prospects," AAPPS Bull., Vol. 18, pp. 33-40, 2008.

[452] S. Fusil, V. Garcia, A. Barthélémy, M. Bibes, "Magnetoelectric devices for spintronics," Annu. Rev. Mater. Res., Vol. 44, p. 91-116, 2014.

[453] Y.W. Yin et al. "Multiferroic tunnel junctions and ferroelectric control of magnetic state at interface," J. Appl. Phys., Vol. 117, Art. No. 172601, 2015.

[454] M. Bibes, A. Barthélémy, "Towards a magnetoelectric memory," Nat. Mater., Vol. 7, pp. 425-426, 2008. 
[455] T. Kosub et al., "Purely antiferromagnetic magnetoelectric random access memory," Nat. Commun. Vol. 8, Art. No. 13985, 2017.

[456] P.K. Amiri et al., "Electric-Field-Controlled Magnetoelectric RAM: Progress, Challenges, and Scaling," IEEE Trans. Magn., Vol. 51, Art. No. 3401507, 2015.

[457] A. Klimov, N. Tiercelin, Y. Dusch, S. Giordano, T. Mathurin, P. Pernod, V. Preobrazhensky, A. Churbanov, S. Nikitov, "Magnetoelectric write and read operations in a stress-mediated multiferroic memory cell," Appl. Phys. Lett., Vol. 110, Art. No. 222401, 2017.

[458] J.-M. Hu, T. Nan, N.X. Sun and L.-Q. Chen, "Multiferroic magnetoelectric nanostructures for novel device applications," MRS Bull., Vol. 40, pp. 728-735, 2015.

[459] J. Shen, J. Cong, Y. Chai, D. Shang, S. Shen, K. Zhai, Y. Tian, Y. Sun, "Nonvolatile Memory Based on Nonlinear Magnetoelectric Effects," Phys. Rev. Appl., Vol. 6, Art. No. 021001, 2016.

[460] A. Chen, Y. Wen, B. Fang, Y. Zhao, Q. Zhang, Y. Chang, P. Li, H. Wu, H. Huang, Y. Lu, Z. Zeng, J. Cai, X. Han, T. Wu, X.-X. Zhang, Y. Zhao, "Giant nonvolatile manipulation of magnetoresistance in magnetic tunnel junctions by electric fields via magnetoelectric coupling," Nat. Commun., Vol. 10, p. 243, 2019.

[461] R. Mishra, D. Kumar, H. Yang, "Oxygen-Migration-Based Spintronic Device Emulating a Biological Synapse," Phys. Rev. Appl. 11, 054065 (2019).

[462] P. Park, C. S. Kim, M. Y. Park, S. Do Kim, and H. K. Yu, "Variable inductance multilayer inductor with MOSFET switch control," IEEE Electron Dev. Lett., vol. 25, no. 3, pp. 144-146, 2004.

[463] M. Vroubel, Y. Zhuang, B. Rejaei, and J. N. Burghartz, "Integrated tunable magnetic RF inductor," IEEE Electron Dev. Lett., vol. 25, no. 12, pp. 787-789, 2004.

[464] W. S. Ishak and K.-W. Chang, "Tunable microwave resonators using magnetostatic wave in YIG films," IEEE Microwave Theory and Tech., vol. 34, no. 12, pp. 1383-1393, 1986.

[465] J. Uher and W. J. Hoefer, "Tunable microwave and millimeter-wave band-pass filters," IEEE Microw. Theory and Tech., vol. 39, no. 4, pp. 643-653, 1991.

[466] C. Tsai et al., "Tunable wideband microwave band-stop and band-pass filters using YIG/GGG-GaAs layer structures," IEEE Trans. Magn., vol. 41, no. 10, pp. 3568-3570, 2005.

[467] K. Entesari and G. M. Rebeiz, "A differential 4-bit 6.5-10-GHz RF MEMS tunable filter IEEE Microw. Theory and Tech., vol. 53, no. 3, pp. 1103-1110, 2005.

[468] V. Sekar, M. Armendariz, and K. Entesari, "A 1.2-1.6-GHz substrate-integrated-waveguide RF MEMS tunable filter," IEEE Microwave Theory and Tech., vol. 59, no. 4, pp. 866-876, 2011.

[469] J. Wu, X. Yang, S. Beguhn, J. Lou, and N. X. Sun, "Nonreciprocal tunable low-loss bandpass filters with ultra-wideband isolation based on magnetostatic surface wave," IEEE Microw. Theory and Tech., vol. 60, no. 12, pp. 3959-3968, 2012.

[470] V. G. Harris, "Modern microwave ferrites," IEEE Trans. Magn., vol. 48, no. 3, pp. 1075-1104, 2011. 
[471] C. Tu, C. Dong, Z. Chu, H. Chen, X. Liang, and N. X. Sun, "A passive isolator realized by magnetoelectric laminate composites," Appl. Phys. Lett., vol. 113, no. 26, p. 262904, 2018.

[472] B. D. Tellegen, "The gyrator, a new electric network element," Philips Res. Rep, vol. 3, no. 2, pp. 81-101, 1948.

[473] S. Dong, J. Zhai, J. Li, D. Viehland, and M. I. Bichurin, "Magnetoelectric gyration effect in $\mathrm{Tb}_{(1-x)} \mathrm{Dy}_{\mathrm{x}} \mathrm{Fe}_{(2-\mathrm{y})} / \mathrm{Pb}(\mathrm{Zr}, \mathrm{Ti}) \mathrm{O}_{3}$ laminated composites at the electromechanical resonance," Appl. Phys. Lett., vol. 89, no. 24, Art. No. 243512, 2006. 


\section{Authors information}

Xianfeng Liang received the B.S. degree in Physics from Nanjing University, Nanjing, China, in 2015. He is currently pursuing the Ph.D. degree in Electrical and Computer Engineering at Northeastern University, Boston, MA, USA. From 2015 to 2020, he was a Research Assistant in Prof. Nian Sun's group at Northeastern University, Boston, MA, USA. His research interests include the development of soft magnetostrictive materials for RF/microwave devices, MEMS applications, giant magnetoimpedance (GMI) MEMS sensors, and MEMS magnetoelectric (ME) antennas and sensors.

Patrick Hayes received his PhD degree in materials science and engineering from Kiel University, Germany, in 2020. He is currently with the Inorganic Functional Materials Group of Dr.-Ing. E. Quandt. His research interests are composite magnetoelectrics, device physics and magnetostrictive materials. His major focus is the exploitation of the converse magnetoelectric effect in thin film composite magnetoelectrics for low-noise biomagnetic sensing applications.

Viktor Schell received the B.Sc. degree in materials science and the M.Sc. degree in materials science and engineering from Kiel University, Germany, in 2014 and 2017, respectively. He is currently pursuing the Ph.D. degree with the Inorganic Functional Materials group at Kiel University as part of the Collaborative Research Centre 1261. His main research interest is the development of highly sensitive and low-noise surface acoustic wave magnetic field sensors for biomedical applications.

Alexei Matyushov received his B.S. and B.A. degrees in physics and political science, respectively, from Arizona State University, Tempe, AZ, USA, in 2010, and his Ph.D. degree in physics from Northeastern University, Boston, MA, USA, in 2020. His dissertation work focused on nanoelectromechanical (NEMS) magnetic sensors made of magnetoelectric composites and operating on the $\Delta \mathrm{E}$ effect. In the fall of 2019, he performed part of his research at Kiel University in Kiel, Germany as a research fellow sponsored by the Penn State-American Ceramic Society-Kiel University (PACK) International Research Experience Fellowship.

Cunzheng Dong received the B.Eng. degree in measuring and control technology and instruments from Tianjin University, Tianjin, China, in 2012, and the M.S. degree in electrical engineering from Northeastern University, Boston, USA, in 2015. He is currently pursuing the Ph.D. degree in the Advanced Materials and Microsystems Laboratory at Northeastern University with Prof. Nian X. Sun. His main research interests include magnetostrictive thin-films, magnetic sensors for biomagnetic sensing, and miniaturized mechanical antennas for VLF communications.

Huaihao Chen received the B.S. degree in microelectronics from Fudan University, Shanghai, China, in 2012 and the M.S. degree in electrical and computer engineering from Northeastern University, Boston, USA, in 2015. He is currently pursuing the Ph. D degree at Northeastern University, Boston, MA, USA. His research interests include magnetic materials and the 
application on integrated MEMS RF devices, design, simulation and fabrication of RF electronics, magnetoelectric antenna and sensors.

Yifan He received the B.E. degree in electrical engineering from Tianjin University, Tianjin, China, in 2014 and the M.S. degree in electrical and computer engineering from Northeastern University, Boston, MA, in 2016. He is currently working toward the Ph.D. degree in electrical engineering at Northeastern University, Boston, MA. His research interest includes magnetic material and its application in RF/microwave devices, energy harvesting devices.

Alexandria Will-Cole received a B.A. degree in Physics from the University of Arizona, Tucson, AZ, USA in 2015 and a M.S. degree in Materials Science and Engineering from Drexel University, Philadelphia, PA, USA in 2019. She is currently a Ph.D. candidate in Electrical Engineering at Northeastern University. Her research interests include the study of structure- and defect-driven property limits in novel ferroelectric and ferromagnetic thin film materials for applications in magnetoelectric devices. She is a National Defense Science and Engineering Graduate Fellow sponsored by the USA Office of Naval Research.

Eckhard Quandt received the Diploma and Dr.-Ing. degrees in physics from the Technische Universität Berlin, Germany, in 1986 and 1990, respectively. He is currently a Professor with Kiel University, Kiel, Germany, where he is also the spokesperson of the DFG CRC 1261 Magnetoelectric Sensors: From Composite Materials to Biomagnetic Diagnostics, and a member of Acatech, National Academy of Science and Engineering.

His scientific focus is material research on smart materials and multiferroics and the development of sensors and actuators for microelectromechanical systems and nanoelectromechanical systems using such materials.

Pedro Martins was born in Guimarães, Portugal, in 1983. He received the graduation degree in physics and chemistry and the Ph.D. degree in physics from the University of Minho (in collaboration with Basque Country University, Spain; and Cambridge University, UK), Braga, in 2006 and 2012, respectively. He is currently an Associate Researcher on the Physics Department of Minho University, since 2019, and is responsible for student coordination (over 20 master students and 6 Ph.D.). From 2012 to 2014 he was also Associate Researcher at the INL International Iberian Nanotechnology Laboratory. He has co-authored over 100 publications (ORCID ID: 0000-0002-9833-9648), collaborates with the Basque Country University, Spain; Wollongong University, Australia and Cambridge University, United Kingdom, among others, and his current research interests include magnetoelectric materials, electroactive materials, printing of smart materials, and device solutions for the loT and 4.0 industries.

Jeffrey McCord received his engineering diploma and Ph.D. degree from the University of Erlangen-Nürnberg, Germany, both in Materials Science and Engineering, in 1992 and 1997.

From 1997 to he was with IBM Storage Division, San Jose, USA, developing magnetic recording head materials. From 2002 to 2009 he was research scientist and group leader at the Leibniz Institute for Solid State Physics and Materials Science, Dresden, Germany. From 2010 to 2011 he was heading the department "Nanomagnetism" at the Helmholtz-Center Dresden-Rossendorf, 
Dresden, Germany. He is co-founder, partner, and served as director of evico magnetics GmbH, Germany. Prof. McCord has joined Kiel University, Germany as a Heisenberg Professor for "Nanomagentism and Magnetic Domains" in the department of Materials Science in 2011. He has authored over 170 papers in peer-reviewed journals in the research field of magnetic films and magnetic domains, and has more than 80 invited talks. He holds several patents on magnetic thin technology. He served in the organization of various IEEE conferences, including as a program co-chair for the Intermag 2017.

Marisa Medarde obtained her PhD in Physics at the University of Barcelona, Spain, working on the application of advanced neutron diffraction, symmetry analysis and $\mathrm{x}$-ray absorption techniques to the investigation of magnetic transition metal oxides. After a post-doc at the Paul Scherrer Institute (PSI) in Villigen, Switzerland and two years at the Argonne National Laboratory in the USA she returned to Switzerland, where she leads the Physical Properties of Materials Group at the Research with Neutrons and Muons division of the PSI. Presently, her scientific activity is focused in the development of new highly correlated oxide materials of interest in fundamental condensed matter or with novel functional properties. Examples of such materials are magnetoelectric multiferroics, materials with multiple order parameters, and complex oxides at the boundary between itinerant and localized behavior. Marisa Medarde served in the Comission on Structure and Dynamics of Condensed Matter (C10) of the International Union of Pure and Applied Physics (IUPAP), and is presently member of the Board of the Swiss Physical Society.

Senentxu Lanceros-Mendez graduated in physics at the University of the Basque Country, Leioa, Spain. He obtained his Ph.D. degree at the Institute of Physics of the Julius-MaximiliansUniversität Würzburg, Germany. He was Research Scholar at Montana State University, Bozeman, MT, USA and visiting scientist at the Pennsylvania State University, USA and University of Potsdam, among others. From 2012 to 2014 he was also Associate Researcher at the INL International Iberian Nanotechnology Laboratory. He is Ikerbasque Professor and Scientific Director at the BCMaterials -Basque Center for Materials, Applications and Nanostructures, Spain and Associated Professor at the Physics Department of the University of Minho, Portugal (on leave). His work is focused on the development of active, smart and (multi)functional materials for sensors and actuators, energy, environmental and biomedical applications. He has co-authored over 600 publications and several patents, co-edited 4 books, and 4 spin-off companies have been developed from his group.

Sebastiaan van Dijken was born in Velsen, the Netherlands, in 1973. He received the M.S. and Ph.D. degrees in applied physics from the University of Twente, Enschede, the Netherlands, in 1996 and 2000, respectively. He was a Post-Doctoral Researcher with the IBM Almaden Research Center, San Jose, CA, USA, from 2000 to 2002. He then worked as a Senior Researcher at Trinity College, Dublin, Ireland, from 2002 to 2006 and the VTT Technical Research Center of Finland, Espoo, Finland, from 2007 to 2008. In 2008, he was appointed as a Full Professor of physics at Aalto University, Espoo, Finland, where he is currently heading the Nanomagnetism and Spintronics group. He has co-authored 180 research articles and 7 books. His current research interests include magnonics, electric-field control of magnetism, magnetoplasmonics, artificial 
spin ice, skyrmionics, and bioinspired multisensory neural networks. Dr. Sebastiaan van Dijken was a recipient of the Finnish Cultural Foundation Prize in 2009, the Väisälä Prize in Physics in 2012, and the Millennium Distinction Award of the Technology Academy Finland in 2015.

Nian Sun is a professor of Electrical and Computer Engineering, and in affiliation with Bioengineering, Director of the W.M. Keck Laboratory for Integrated Ferroics, Northeastern University, and founder and chief technology advisor of Winchester Technologies, LLC. He received his Ph.D. degree from Stanford University. Prior to joining Northeastern University, he was a Scientist at IBM and Hitachi Global Storage Technologies. Dr. Sun was the recipient of the Humboldt Research Award, NSF CAREER Award, ONR Young Investigator Award, Outstanding Research Award, Outstanding Translational Research Award, etc.

His research interests include novel magnetic, ferroelectric, and multiferroic materials, devices, and microsystems, novel gas sensors, and sensor systems, etc. He has over 280 publications and over 30 patents and patent applications. One of his papers was selected as the "Ten most outstanding full papers in the past decade (2001 2010) in Advanced Functional Materials". He is a fellow of the IEEE, the Institute of Physics, and the Institution of Engineering and Technology, and an editor of IEEE Transactions on Magnetics, and Sensors Journal.

Jordi Sort leads the 'Group of Smart Nanoengineered Materials, Nanomechanics and Nanomagnetism' (with around 20 researchers) as an ICREA Research Professor at UAB. After finishing his PhD in 2002 in the field of "magnetic interfacial effects" (Extraordinary Award), Prof. Sort performed two postdoctoral stays, at SPINTEC (Grenoble) and at Argonne National Laboratory. His research is focused on a wide variety of materials (thin films, lithographed structures, porous materials and nanocomposites) with emphasis on their magnetic and mechanical performance. He received awards from the Catalan and Spanish Physical Societies, UPC and Naturgy, as well as the Federation of European Materials Societies. He is also Fellow of the Institute of Physics, Editor-in-Chief of the Section "Nanocomposite Materials" in the journal Nanomaterials (MDPI) and Associate Editor of APL Materials (AIP). At present, Prof. Sort has supervised 15 PhD Theses, has published more than 320 articles (above 9000 citations in ISI WoS, $h=46)$, has issued 6 patents and has managed 31 national/international research projects, being Coordinator of two European Training Network (ITN) and a Consolidator Grant and a Proof of Concept from the European Research Council. 